\title{
On the boundary theory of subordinate killed Lévy processes
}

\author{
Panki Kim* Renming Song ${ }^{\dagger}$ and Zoran Vondraček $\ddagger$
}

\begin{abstract}
Let $Z$ be a subordinate Brownian motion in $\mathbb{R}^{d}, d \geq 2$, via a subordinator with Laplace exponent $\phi$. We kill the process $Z$ upon exiting a bounded open set $D \subset$ $\mathbb{R}^{d}$ to obtain the killed process $Z^{D}$, and then we subordinate the process $Z^{D}$ by a subordinator with Laplace exponent $\psi$. The resulting process is denoted by $Y^{D}$. Both $\phi$ and $\psi$ are assumed to satisfy certain weak scaling conditions at infinity.

We study the potential theory of $Y^{D}$, in particular the boundary theory. First, in case that $D$ is a $\kappa$-fat bounded open set, we show that the Harnack inequality holds. If, in addition, $D$ satisfies the local exterior volume condition, then we prove the Carleson estimate. In case $D$ is a smooth open set and the lower weak scaling index of $\psi$ is strictly larger than $1 / 2$, we establish the boundary Harnack principle with explicit decay rate near the boundary of $D$. On the other hand, when $\psi(\lambda)=\lambda^{\gamma}$ with $\gamma \in(0,1 / 2]$, we show that the boundary Harnack principle near the boundary of $D$ fails for any bounded $C^{1,1}$ open set $D$. Our results give the first example where the Carleson estimate holds true, but the boundary Harnack principle does not.

One of the main ingredients in the proofs is the sharp two-sided estimates of the Green function of $Y^{D}$. Under an additional condition on $\psi$, we establish sharp twosided estimates of the jumping kernel of $Y^{D}$ which exhibit some unexpected boundary behavior.

We also prove a boundary Harnack principle for non-negative functions harmonic in a smooth open set $E$ strictly contained in $D$, showing that the behavior of $Y^{D}$ in the interior of $D$ is determined by the composition $\psi \circ \phi$.
\end{abstract}

AMS 2010 Mathematics Subject Classification: Primary 60J45; Secondary 60J50, $60 \mathrm{~J} 75$.

Keywords and phrases: Lévy processes, subordination, Green functions, jumping kernels, harmonic functions, Harnack inequality, boundary Harnack principle, Carleson estimate.

\section{Introduction}

The fractional Laplacian $(-\Delta)^{\alpha}, \alpha \in(0,1)$, in $\mathbb{R}^{d}, d \geq 1$, is a well-studied object in various branches of mathematics. There are many definitions of this operator as an operator on

*This work was supported by the National Research Foundation of Korea (NRF) grant funded by the Korea government(MSIP) (No. 2016R1E1A1A01941893).

${ }^{\dagger}$ Research supported in part by a grant from the Simons Foundation (\#429343, Renming Song).

${ }^{\ddagger}$ Research supported in part by the Croatian Science Foundation under the project 3526 . 
the Lebesgue spaces or the space of continuous functions. A detailed discussion of different definitions of the fractional Laplacian and their equivalence are given in the recent paper [20]. From a probabilistic point of view, the fractional Laplacian is the infinitesimal generator of the semigroup of the isotropic $2 \alpha$-stable process. To be more precise, let $X=\left(X_{t}, \mathbb{P}_{x}\right)$ be an isotropic $2 \alpha$-stable process in $\mathbb{R}^{d}, \alpha \in(0,1)$. For any non-negative (or bounded) Borel function $f: \mathbb{R}^{d} \rightarrow \mathbb{R}$ and $t \geq 0$, let $Q_{t} f(x):=\mathbb{E}_{x} f\left(X_{t}\right)$. Then

$$
-(-\Delta)^{\alpha} f=\lim _{t \rightarrow 0} \frac{Q_{t} f-f}{t}
$$

provided the limit exists (in the appropriate function space).

Another definition of the $\alpha$-fractional Laplacian with a probabilistic flavor is through Bochner's subordination of semigroups (or Bochner's functional calculus). Let $\Delta$ be the standard Laplacian defined on some function space, $\left(P_{t}\right)_{t \geq 0}$ the corresponding semigroup, and $\chi(\lambda)=\lambda^{\alpha}, \lambda>0$. Then

$$
-(-\Delta)^{\alpha} f=-\chi(-\Delta) f=\frac{1}{|\Gamma(-\alpha)|} \int_{0}^{\infty}\left(P_{t} f-f\right) t^{-\alpha-1} d t .
$$

The probabilistic interpretation is as follows: Let $W=\left(W_{t}, \mathbb{P}_{x}\right)$ be a Brownian motion in $\mathbb{R}^{d}$ and $S=\left(S_{t}\right)_{t \geq 0}$ an independent $\alpha$-stable subordinator. Then the subordinate process $\left(W_{S_{t}}\right)_{t \geq 0}$ is an isotropic $2 \alpha$-stable process, and (1.1) and (1.2) are exactly the same.

Once we move from the whole of $\mathbb{R}^{d}$ to an open subset $D \subset \mathbb{R}^{d}$, the question of defining a fractional Laplacian in $D$ becomes more delicate. From a probabilistic point of view, there are two obvious choices. The first, and the most common one, is to consider the isotropic $2 \alpha$-stable process $X$ killed upon exiting $D$. More precisely, let $\tau_{D}^{X}:=\inf \left\{t>0: X_{t} \notin D\right\}$, and set $X_{t}^{D}:=X_{t}$ if $t<\tau_{D}^{X}$, and $X_{t}:=\partial$ if $t \geq \tau_{D}^{X}$ (here $\partial$ is an extra point usually called the cemetery). Define the corresponding semigroup $\left(Q_{t}^{D}\right)_{t \geq 0}$ by $Q_{t}^{D} f(x):=\mathbb{E}_{x} f\left(X_{t}^{D}\right)=$ $\mathbb{E}_{x}\left(f\left(X_{t}\right), t<\tau_{D}^{X}\right)$. Let

$$
\mathcal{L}_{1} f:=\lim _{t \rightarrow 0} \frac{Q_{t}^{D} f-f}{t}
$$

be the infinitesimal generator of $\left(Q_{t}^{D}\right)_{t \geq 0}$. This is one possible definition of an $\alpha$-fractional Laplacian in $D$. It is usually called the fractional Laplacain in $D$ with zero exterior condition and can be denoted by $-(-\Delta)_{\mid D}^{\alpha}$. This definition corresponds to the definition in (1.1). The second possible choice for an $\alpha$-fractional Laplacian in $D$ is to apply Bochner's functional calculus to the Dirichlet Laplacian $\Delta_{\mid D}$. To be more precise, let $\left(P_{t}^{D}\right)_{t \geq 0}$ be the semigroup corresponding to the killed Brownian motion $W^{D}$, and let $\Delta_{\mid D}$ be the infinitesimal generator of this semigroup. Set

$$
\mathcal{L}_{0} f:=-\left(-\Delta_{\mid D}\right)^{\alpha} f=-\chi\left(-\Delta_{\mid D}\right) f=\frac{1}{|\Gamma(-\alpha)|} \int_{0}^{\infty}\left(P_{t}^{D} f-f\right) t^{-\alpha-1} d t
$$

This definition corresponds to the one in (1.2), but it yields an operator which is different from $\mathcal{L}_{1}$. Probabilistically, $\mathcal{L}_{0}$ is the infinitesimal generator of the semigroup $\left(\widetilde{Q}_{t}^{D}\right)_{t \geq 0}$ corresponding to the subordinate process $\left(W_{S_{t}}^{D}\right.$ ) (where $S$ is still an $\alpha$-stable subordinator independent of $W)$. The semigroup $\left(\widetilde{Q}_{t}^{D}\right)_{t \geq 0}$ is subordinate to the semigroup $\left(Q_{t}^{D}\right)_{t \geq 0}$ in the sense that $\widetilde{Q}_{t}^{D} f(x) \leq Q_{t}^{D} f(x)$ for all non-negative $f$ and all $x \in D$. 
Note that $\mathcal{L}_{0}$ and $\mathcal{L}_{1}$ are infinitesimal generators of processes that are obtained from the Brownian motion $W$ by using two operations: killing and subordination. For $\mathcal{L}_{0}$ we first kill the Brownian motion when it exits $D$ and then subordinate it via the subordinator $S$. For $\mathcal{L}_{1}$ the order is reversed: we first subordinate $W$ to get $X$, and then kill $X$ when it exits $D$. This interpretation suggests that $\mathcal{L}_{0}$ and $\mathcal{L}_{1}$ are just two extremal possibilities for an infinite choice of fractional Laplacians in $D$. For example, let $\gamma, \delta \in(0,1)$ be such that $\delta \gamma=\alpha$. Let $Z_{t}:=W_{S_{t}}$ be a subordinate Brownian motion where now $S$ is an independent $\delta$-stable subordinator. Note that $Z$ is an isotropic $2 \delta$-stable process. Let $Z^{D}$ be the process $Z$ killed upon exiting $D$, and let $Y_{t}^{D}:=Z_{T_{t}}^{D}$ be the subordinate process via an independent $\gamma$-stable subordinator $T$. Denote by $X$ the (twicely) subordinate Brownian motion: $X_{t}=Z_{T_{t}}=$ $W\left(S_{T_{t}}\right)$, and note that $X$ is a $2 \alpha$-stable process. If $\left(R_{t}^{D}\right)_{t \geq 0}$ denotes the semigroup of $Y^{D}$, then $\widetilde{Q}_{t}^{D} f(x) \leq R_{t}^{D} f(x) \leq Q_{t}^{D} f(x)$ for all non-negative $f$ and all $x \in D$. The infinitesimal generator $\mathcal{L}$ of the semigroup $\left(R_{t}^{D}\right)$ can be written as

$$
\mathcal{L}:=-\left((-\Delta)^{\delta}{ }_{\mid D}\right)^{\gamma}
$$

and since $\delta \gamma=\alpha$, it also has the right to be called an $\alpha$-fractional Laplacian on $D$.

The purpose of this paper is to study the potential theory of the operators as defined in the display above and see how their properties depend on $\delta$ and $\gamma$. When $\delta=1$ and $\gamma \in(0,1)$ (so $\alpha=\gamma), Y^{D}$ reduces to a subordinate killed Brownian motion. This case was recently studied in [18] where it was shown that the boundary behavior of $Y^{D}$ is roughly the same as that of the killed Brownian motion in $D$, while in the interior of $D, Y^{D}$ behaves like a $2 \alpha$-stable process. In the current case, namely $\delta \in(0,1)$, we will show that, in the interior of $D$, the process $Y^{D}$ still behaves like a $2 \alpha$-stable process. Two potential-theoretic justifications of this are (i) the Green function interior estimates given in Proposition 3.7, and (ii) the scale invariant boundary Harnack principle, Theorem 4.10, which implies that the boundary behavior of non-negative functions which are harmonic in an open set $E \subset \bar{E} \subset D$ is the same as for the $2 \alpha$-stable process.

On the other hand, the boundary potential theory of $Y^{D}$ is much more complicated and depends on the range of $\gamma$. In order to explain the intricacies involved, we first recall the statement of the boundary Harnack principle (BHP). Let $D$ be a bounded open set in $\mathbb{R}^{d}$. We say that the BHP holds for $Y^{D}$ if there exists $\widehat{R}>0$ such that for every $r \in(0, \widehat{R}]$, there exists a constant $c_{r} \geq 1$ such that for every $Q \in \partial D$ and any two non-negative functions $f$ and $g$ defined on $D$ which are harmonic in $D \cap B(Q, r)$ and vanish continuously on $\partial D \cap B(Q, r)$, it holds that

$$
\frac{f(x)}{g(x)} \leq c_{r} \frac{f(y)}{g(y)}, \quad x, y \in D \cap B(Q, r / 2) .
$$

If the constant $c_{r}$ above can be chosen independently of $r \in(0, \widehat{R}]$, we say that the scale invariant BHP holds.

Usually, to prove the BHP one first establishes sharp two-sided Green function estimates and the Carleson estimate. This is the road we also take. As our first main result, we prove the Carleson estimate for rather rough open sets, see Theorem 5.5 for the precise statement. The Green function estimates in an arbitrary bounded $C^{1,1}$ open set $D$ are given in Theorem 6.4. The Green function estimates provide indication for the decay rate of non-negative harmonic functions near the boundary of $D$.

Our second main result concerns the case $\gamma \in(1 / 2,1)$. In this case we prove a scale invariant BHP with the explicit decay rate, cf. Theorem [7.5, namely there exist constants 
$\widehat{R}>0$ and $c \geq 1$, such that for every $r \in(0, \widehat{R}]$, every $Q \in \partial D$ and any non-negative function $f$ defined on $D$ which is harmonic in $D \cap B(Q, r)$ and vanishes continuously on $\partial D \cap B(Q, r)$, it holds that

$$
\frac{f(x)}{\operatorname{dist}(x, \partial D)^{\delta}} \leq c \frac{f(y)}{\operatorname{dist}(y, \partial D)^{\delta}}, \quad x, y \in D \cap B(Q, r / 2) .
$$

Our last main result, and the most interesting one, is about the case $\gamma \in(0,1 / 2]$. Here we show that even the non-scale invariant BHP does not hold in any bounded $C^{1,1}$ open set, cf. Section 9. We accomplish this by constructing a sequence of harmonic functions that, in the limit, have different decay rate at the boundary than that of the Green function. One of the possible reasons for the failure of the BHP is that the jumping kernel of $Y^{D}$ exhibits some unexpected boundary behavior which indicates a sort of phase transition at $\gamma=1 / 2$. This boundary behavior is very different from that of the jumping kernel of the subordinate killed Brownian motion studied in [18].

To the best of our knowledge, our results give the first example where the Carleson estimate holds, but the BHP fails. It was shown in [25, Theorem 4.3] that when $D$ is a bounded $\kappa$-fat open set, the Martin boundary and the minimal Martin boundary of $Y^{D}$ both coincide with the Euclidean boundary $\partial D$ for all $\gamma \in(0,1)$. This makes the failure of the BHP in the case $\gamma \in(0,1 / 2]$ somewhat a surprise to us.

In [11] and [13], it has been shown that the (non-scale invariant) BHP does not hold in a certain $C^{1,1}$ domain (connected open set) $D$ for truncated stable processes and subordinate Brownian motions with Gaussian components respectively. In both cases this was accomplished by showing the Carleson estimate fails in $D$. For an extensive survey of the literature on BHP, see the introduction of [17].

The paper is written for much more general subordinators than $\delta$ and $\gamma$-stable ones. The subordinators we consider are defined through their Laplace exponents $\phi$ and $\psi$ which are assumed to satisfy certain weak scaling conditions at infinity. The precise setting and the background material are given in Section 2. In Section 3 we show that the Green functions of small sets away from the boundary of $D$ with respect to the processes $Y^{D}$ and $X$ are comparable. The argument uses a non-local Feynman-Kac transform of $X$, which was already employed in [18. The main results of Section 4 are the Harnack inequality for nonnegative functions harmonic with respect to $Y^{D}$, Theorems 4.7 and 4.8, and the boundary Harnack principle for non-negative functions harmonic in an open set strictly contained in $D$, Theorem 4.10, in case when $D$ is $\kappa$-fat. The proof of the latter is only sketched, being similar to the proof of the corresponding result in [18, while the proof of the former is different. In Section 5 we establish the Carleson estimate in bounded $\kappa$-fat open sets satisfying the local exterior volume condition by using a parabolic version of the Carleson estimate for the process $Z$, cf. Proposition 5.4. The main result of Section 6 is the sharp two-sided estimates on the Green function of $Y^{D}$ in bounded $C^{1,1}$ open sets, cf. Theorem 6.4. In Section 7 we prove the boundary Harnack principle with explicit decay rate in an arbitrary bounded $C^{1,1}$ open set $D$ for non-negative functions harmonic in a neighborhood of a boundary point of $D$ under the assumption that the lower weak scaling index of $\psi$ is strictly larger than $1 / 2$. In the proofs of the Carleson estimate and the boundary Harnack principle we follow the ideas from [18], but contrary to that paper we do not use the explicit boundary behavior of the jumping kernel. In Section 8 we address the question of the boundary behavior of the jumping kernel of $Y^{D}$ in bounded $C^{1,1}$ open sets. Here we need to distinguish between two 
cases, essentially corresponding to $\gamma$-stable subordinators with $\gamma<1 / 2$ and $\gamma>1 / 2$. In these two cases the jumping kernels exhibit different boundary behavior, and there seems to be some sort of phase transition going from one to the other case. The main result there is Theorem 8.4, while Example 8.5 gives the full picture for stable subordinators. Finally, in the last section we show that, in case $\psi(\lambda)=\lambda^{\gamma}$ with $\gamma \in(0,1 / 2]$, the BHP does not hold for any bounded $C^{1,1}$ open set.

In the remainder of this paper, unless indicated otherwise, whenever we mention the boundary Harnack principle, we mean the scale invariant one.

We end this introduction with a few words about notation used throughout the paper. For any two positive functions $f$ and $g$ and constant $c \geq 1, f \asymp^{c} g$ means that $c^{-1} g \leq f \leq c g$ on their common domain of definition. We will use ":=" to denote a definition, which is read as "is defined to be". For $a, b \in \mathbb{R}, a \wedge b:=\min \{a, b\}$ and $a \vee b:=\max \{a, b\}$. For any $x \in \mathbb{R}^{d}$ and $r>0$, we use $B(x, r)$ to denote the open ball of radius $r$ centered at $x$. For a Borel set $V$ in $\mathbb{R}^{d},|V|$ denotes the Lebesgue measure of $V$. For any open set $U \subset \mathbb{R}^{d}$ and $x \in \mathbb{R}^{d}$, we use $\delta_{U}(x)$ to denote the distance between $x$ and the boundary $\partial U$. For any process $\left(X_{t}\right)_{t \geq 0}$, we sometimes write $X(t)$ instead of $X_{t}$ for notational simplicity. Upper case letters $C$ 's without subscripts denote strictly positive constants in the statements of results and their values may change in each result. Upper case letters with subscripts $C_{i}, i=0,1,2, \ldots$, denote constants that will be fixed throughout the paper. Lower case letters $c$ 's without subscripts denote strictly positive constants whose values are unimportant and which may change from line to line, while values of lower case letters with subscripts $c_{i}, i=0,1,2, \ldots$, are fixed in each proof, and the labeling of these constants starts anew in each proof. $c_{i}=c_{i}(a, b, c, \ldots)$, $i=0,1,2, \ldots$, denote constants depending on $a, b, c, \ldots$ The dependence on the dimension $d \geq 1$ may not be mentioned explicitly. For the exit times from a Borel set $U$ of the three processes, we use notation $\tau_{U}^{Z}:=\inf \left\{t>0: Z_{t} \notin U\right\}, \tau_{U}^{X}:=\inf \left\{t>0: X_{t} \notin U\right\}$, and $\tau_{U}:=\inf \left\{t>0: Y_{t}^{D} \notin U\right\}$.

\section{Preliminaries}

Let $W=\left(W_{t}, \mathbb{P}_{x}\right)_{t \geq 0, x \in \mathbb{R}^{d}}$ be a Brownian motion in $\mathbb{R}^{d}$ running twice as fast as the standard Brownian motion, $d \geq 2$, and $S=\left(S_{t}\right)_{t \geq 0}$ an independent subordinator with Laplace exponent $\phi$ and Lévy measure $\mu$. We assume that $\phi$ is a complete Bernstein function satisfying the following weak scaling condition at infinity: There exist $a_{1}, a_{2}>0$ and $0<\delta_{1} \leq \delta_{2}<1$ such that

$$
a_{1}\left(\frac{R}{r}\right)^{\delta_{1}} \leq \frac{\phi(R)}{\phi(r)} \leq a_{2}\left(\frac{R}{r}\right)^{\delta_{2}}, \quad 1<r \leq R<\infty .
$$

It is clear that, for any $r_{0} \in(0,1)$, (2.1) is still valid for $r_{0}<r \leq R<\infty$ with constants $a_{1}, a_{2}$ depending on $r_{0}, \delta_{1}$ and $\delta_{2}$. We will implicitly use this throughout the paper and will write $a_{1}\left(r_{0}\right)$ and $a_{2}\left(r_{0}\right)$ for the corresponding constants. The same remark also applies to (2.2), (2.3) and (2.5) below. Without loss of generality we also assume that $\phi(1)=1$. Note that it follows from the right-hand side inequality in (2.1) that $\phi$ has no drift.

Define $Z=\left(Z_{t}\right)_{t \geq 0}$ by $Z_{t}:=W\left(S_{t}\right)$. Then $Z$ is an isotropic Lévy process with characteristic exponent $\xi \mapsto \bar{\phi}\left(|\xi|^{2}\right)$ and it is called a subordinate Brownian motion.

Let $T=\left(T_{t}\right)_{t \geq 0}$ be another subordinator, independent of $Z$, with Laplace exponent $\psi$ and Lévy measure $\nu$. We assume that $\psi$ is also a complete Bernstein function satisfying the 
following weak scaling condition at infinity: There exist $b_{1}, b_{2}>0$ and $0<\gamma_{1} \leq \gamma_{2}<1$ such that

$$
b_{1}\left(\frac{R}{r}\right)^{\gamma_{1}} \leq \frac{\psi(R)}{\psi(r)} \leq b_{2}\left(\frac{R}{r}\right)^{\gamma_{2}}, \quad 1<r \leq R<\infty .
$$

So $\psi$ also has no drift. Without loss of generality we also assume that $\psi(1)=1$. Let $X=$ $\left(X_{t}\right)_{t \geq 0}$ be the process obtained by subordinating $Z$ via the subordinator $T: X_{t}:=Z\left(T_{t}\right)$. Since $X_{t}=W\left(S_{T_{t}}\right)$, we see that $X$ is a subordinate Brownian motion via the subordinator $S \circ T$ with Laplace exponent $\psi \circ \phi$. It is straightforward to see that $\psi \circ \phi$ is a complete Bernstein function (see [22, Corollary 7.9(iii)]) satisfying the following weak scaling condition at infinity:

$$
b_{1} a_{1}^{\gamma_{1}}\left(\frac{R}{r}\right)^{\gamma_{1} \delta_{1}} \leq \frac{(\psi \circ \phi)(R)}{(\psi \circ \phi)(r)} \leq b_{2} a_{2}^{\gamma_{2}}\left(\frac{R}{r}\right)^{\gamma_{2} \delta_{2}}, \quad 1<r \leq R<\infty .
$$

Let $D \subset \mathbb{R}^{d}$ be an open set. We define the killed processes $Z^{D}$ and $X^{D}$ in the usual way. Note that both $Z^{D}$ and $X^{D}$ are killed subordinate Brownian motions, and that $X^{D}$ may also be regarded as the process obtained by first subordinating the subordinate Brownian motion $Z$ via $T$ and then killing it upon exiting $D$. We define another process by reversing the order of killing and subordination of $Z$ : Let $Y^{D}=\left(Y_{t}^{D}\right)_{t \geq 0}$ be defined by $Y_{t}^{D}:=Z^{D}\left(T_{t}\right)$. Then $Y^{D}$ is the process obtained by first killing $Z$ upon exiting from $D$ and then subordinating the killed process via $T$. We will use $\left(Q_{t}^{D}\right)_{t \geq 0}$ to denote the semigroup of $X^{D}$ and $\left(R_{t}^{D}\right)_{t \geq 0}$ the semigroup of $Y^{D}$. It was shown in [26] that $Y^{D}$ can be realized as $X^{D}$ killed at a terminal time and that the semigroup $\left(R_{t}^{D}\right)_{t \geq 0}$ is subordinate to the semigroup $\left(Q_{t}^{D}\right)_{t \geq 0}$ in the sense that $R_{t}^{D} f(x) \leq Q_{t}^{D} f(x)$ for all Borel $f: D \rightarrow[0, \infty)$, all $t \geq 0$ and all $x \in D$. As a consequence, cf. [7, Proposition 4.5.2] and [24, Proposition 3.2], we have the following relation between the killing function $\kappa^{Y^{D}}$ of $Y^{D}$ and the killing function $\kappa^{X^{D}}$ of $X^{D}$ :

$$
\kappa^{Y^{D}}(x) \geq \kappa^{X^{D}}(x), \quad x \in D .
$$

Let $v(t)$ be the potential density of the subordinator $T$. Since $\psi$ satisfies (2.2), by [14, Corollary 2.4 and Proposition 2.5], there exists $c \geq 1$ such that

$$
v(t) \asymp^{c} \frac{1}{t \psi\left(t^{-1}\right)}, \quad \nu(t) \asymp^{c} \frac{\psi\left(t^{-1}\right)}{t}, \quad 0<t<1 .
$$

Thus $\nu(t)$ satisfies the doubling property near zero: For every $M>0$ there exists $c=c(M)>$ 0 such that

$$
\nu(t) \leq c \nu(2 t), \quad 0<t \leq M .
$$

Since $\psi$ is a complete Bernstein function, it follows from [12, Lemma 2.1] that there exists $c>0$ such that

$$
\nu(t) \leq c \nu(t+1), \quad t \geq 1 .
$$

Now we list some auxiliary results. First, for simplicity, we let $\Phi(r):=\frac{1}{\phi\left(r^{-2}\right)}$. By concavity and monotonicty of $\phi$, it is clear that

$$
(1 \wedge \lambda) \phi(t) \leq \phi(\lambda t) \leq(1 \vee \lambda) \phi(t), \quad \lambda, t>0
$$


As a consequence,

$$
\left(1 \wedge \lambda^{2}\right) \Phi(t) \leq \Phi(\lambda t) \leq\left(1 \vee \lambda^{2}\right) \Phi(t), \quad \lambda, t>0
$$

Let $\Phi^{-1}$ be the inverse function of $\Phi$. It is shown in [5, (7.2)] that $\Phi^{-1}$ satisfies the following scaling property: For each $T>0$, there exists $C(T) \geq 1$ such that

$$
C(T)^{-1}\left(\frac{r}{R}\right)^{1 / 2 \delta_{1}} \leq \frac{\Phi^{-1}(r)}{\Phi^{-1}(R)} \leq C(T)\left(\frac{r}{R}\right)^{1 / 2 \delta_{2}}, \quad 0<r \leq R \leq T .
$$

Next, since $\psi, \phi$ and $\psi \circ \phi$ are complete Bernstein functions, by [22, Proposition 7.1 and $(7.3)]$

$$
t / \phi(t), t \psi\left(t^{-1}\right), t \phi\left(t^{-1}\right) \text { and } t(\psi \circ \phi)\left(t^{-1}\right) \text { are complete Bernstein functions. }
$$

Hence,

$$
t \mapsto t^{-2} \Phi(t) \quad \text { is a decreasing function, }
$$

and, since $d \geq 2$,

$$
t \mapsto t^{d} \psi\left(\Phi(t)^{-1}\right) \quad \text { is an increasing function. }
$$

Let $p(t, x, y)$ be the transition density of $Z$. Note that $p(t, x, y)=p(t,|x-y|)$ where

$$
p(t, r)=\int_{0}^{\infty}(4 \pi s)^{-d / 2} \exp \left\{-r^{2} / 4 s\right\} \mathbb{P}\left(S_{t} \in d s\right)
$$

is decreasing in $r$. We denote by $p^{D}(t, x, y)$ the transition density of $Z^{D}$, and by $\left(P_{t}^{D}\right)_{t \geq 0}$ the corresponding semigroup. By the strong Markov property, $p^{D}(t, x, y)$ is given by the formula

$$
p^{D}(t, x, y):=p(t, x, y)-\mathbb{E}_{x}\left[p\left(t-\tau_{D}^{Z}, Z\left(\tau_{D}^{Z}\right), y\right), \tau_{D}^{Z}<t\right], \quad t>0, x, y \in D .
$$

Recall that $X$ can be regarded as a subordinate Brownian motion via $S \circ T$. Let $q(t, x, y)$ be the transition density of $X$, and $q^{D}(t, x, y)$ the transition density of $X^{D}$. We will need the upper bound for the Green function $G_{D}^{X}$ of the process $X^{D}$ in case $D$ is bounded. When the process $X$ is transient (which is always true when $d \geq 3$ ), the required upper bound is the standard upper bound for the Green function of $X$. The lemma below covers also the case $d=2$.

Lemma 2.1 Let $D \subset \mathbb{R}^{d}$ be a bounded open set, $d \geq 2$. There exists a constant $c=$ $c(\operatorname{diam}(D)) \geq 1$ such that for all $x, y \in D$,

$$
G_{D}^{X}(x, y) \leq c \frac{1}{|x-y|^{d} \psi\left(\Phi(|x-y|)^{-1}\right)} .
$$

Proof. It follows from [21, Lemma 48.3] that, for any bounded open set $U \subset \mathbb{R}^{d}$, there exists $t_{1}>0$ such that $\sup _{x \in \mathbb{R}^{d}} \mathbb{P}_{x}\left(X_{t_{1}} \in U\right)<1$. Using this and the Chapman-Kolmogorov equation, one can easily show (see, for instance, the proof of [4, Lemma 3.7]) that, for every $R>0$, there exist $c_{1}, c_{2}>0$ such that for every $x_{0} \in \mathbb{R}^{d}$,

$$
q^{B\left(x_{0}, R\right)}(t, x, y) \leq c_{2} e^{-c_{1} t} \quad \text { for all }(t, x, y) \in\left(t_{1}, \infty\right) \times B\left(x_{0}, R\right) \times B\left(x_{0}, R\right)
$$


Using this and the upper bound of $q(t, x, y)$ for $t \in(0,1]$ in [10, Theorem 3] and [19, Proposition 3.2], we immediately get that, for every $R, T>0$, there exist $c_{3}, c_{4}>0$ such that for every $x_{0} \in \mathbb{R}^{d}$ and $x, y \in B\left(x_{0}, R\right)$,

$$
q^{B\left(x_{0}, R\right)}(t, x, y) \leq c_{4} \begin{cases}e^{-c_{3} t} & \text { for all } t \in[T, \infty) \\ {\left[(\psi \circ \phi)^{-1}\left(t^{-1}\right)\right]^{d / 2} \wedge \frac{t(\psi \circ \phi)\left(|x-y|^{-2}\right)}{|x-y|^{d}}} & \text { for all } t \in(0, T)\end{cases}
$$

Let $T_{R}=2 /(\psi \circ \phi)\left((2 R)^{-2}\right)$. Note that using (2.3), the analog of (2.10) for $\psi \circ \phi$, and the fact $d \geq 2$, by the change of variable $u=1 / t(\psi \circ \phi)\left(|x-y|^{-2}\right)$ we have that for $x, y \in B\left(x_{0}, R\right)$,

$$
\begin{aligned}
& |x-y|^{d} \psi\left(\Phi(|x-y|)^{-1}\right) \int_{0}^{T_{R}}\left[(\psi \circ \phi)^{-1}\left(t^{-1}\right)\right]^{d / 2} \wedge \frac{t(\psi \circ \phi)\left(|x-y|^{-2}\right)}{|x-y|^{d}} d t \\
= & \int_{1}^{\infty} u^{-3} d u+\int_{1 / T_{R}(\psi \circ \phi)\left(|x-y|^{-2}\right)}^{1} u^{-2}\left(\frac{(\psi \circ \phi)^{-1}\left(u(\psi \circ \phi)\left(|x-y|^{-2}\right)\right)}{(\psi \circ \phi)^{-1}\left((\psi \circ \phi)\left(|x-y|^{-2}\right)\right)}\right)^{d / 2} d u \\
\leq & c_{5}+c_{6} \int_{0}^{1} u^{-2+d /\left(2 \gamma_{2} \delta_{2}\right)} d u=c_{7}(R)<\infty .
\end{aligned}
$$

Using this and (2.16) we see that

$$
\begin{aligned}
& G_{D}^{X}(x, y) \leq G_{B\left(x_{0}, \operatorname{diam}(D)\right)}^{X}(x, y) \\
= & \int_{0}^{T_{\operatorname{diam}(D)}} q^{B\left(x_{0}, \operatorname{diam}(D)\right)}(t, x, y) d t+\int_{T_{\operatorname{diam}(D)}}^{\infty} q^{B\left(x_{0}, \operatorname{diam}(D)\right)}(t, x, y) d t \\
& \leq c_{8} \int_{0}^{T_{\operatorname{diam}(D)}}\left[(\psi \circ \phi)^{-1}\left(t^{-1}\right)\right]^{d / 2} \wedge \frac{t(\psi \circ \phi)\left(|x-y|^{-2}\right)}{|x-y|^{d}} d t+c_{8} \int_{T_{\operatorname{diam}(D)}}^{\infty} e^{-c_{9} t} d t \\
& \leq c \frac{1}{|x-y|^{d} \psi\left(\Phi(|x-y|)^{-1}\right)}, \quad \text { for all } x, y \in D,
\end{aligned}
$$

thus proving (2.15).

Recall that $Y_{t}^{D}=Z^{D}\left(T_{t}\right)$ is the process obtained by subordinating the process $Z^{D}$ via the independent subordinator $T$. The transition semigroup $\left(R_{t}^{D}\right)_{t \geq 0}$ of $Y^{D}$ admits a transition density given by

$$
r^{D}(t, x, y):=\int_{0}^{\infty} p^{D}(s, x, y) \mathbb{P}\left(T_{t} \in d s\right) .
$$

Since the semigroup $\left(R_{t}^{D}\right)_{t \geq 0}$ is subordinate to the semigroup $\left(Q_{t}^{D}\right)_{t \geq 0}$, when $D$ is a bounded open set, the process $Y^{D}$ is transient and admits a Green function

$$
G^{Y^{D}}(x, y):=\int_{0}^{\infty} r^{D}(t, x, y) d t=\int_{0}^{\infty} p^{D}(t, x, y) v(t) d t
$$

Moreover, for every bounded open set $D$,

$$
G^{Y^{D}}(x, y) \leq G_{D}^{X}(x, y), \quad x, y \in D
$$


Let $J^{Y^{D}}(x, y)$ be the jumping kernel of $Y^{D}$ given by

$$
J^{Y^{D}}(x, y):=\int_{0}^{\infty} p^{D}(t, x, y) \nu(t) d t
$$

and let $J^{X}(x, y):=j^{X}(|x-y|)$ be the Lévy density of $X$ given by

$$
j^{X}(|x-y|):=\int_{0}^{\infty} p(t, x, y) \nu(t) d t
$$

Clearly,

$$
J^{Y^{D}}(x, y) \leq j^{X}(|x-y|), \quad x, y \in D
$$

Furthermore, by [14, Lemma 3.2], for any $M>0$ there exists $c(M) \geq 1$ such that

$$
c(M)^{-1} \frac{\psi\left(\Phi(|x-y|)^{-1}\right)}{|x-y|^{d}} \leq j^{X}(|x-y|) \leq c(M) \frac{\psi\left(\Phi(|x-y|)^{-1}\right)}{|x-y|^{d}}, \quad|x-y| \leq M .
$$

Recall that, for any Borel set $U \subset D$, we use the notation $\tau_{U}=\inf \left\{t>0: Y_{t}^{D} \notin U\right\}$ for the exit time from $U$ of $Y^{D}$.

Since $Y^{D}$ can be realized as $X^{D}$ killed at a terminal time, it follows from [27] that if $U \subset D$ is a Lipschitz open set, then

$$
\mathbb{P}_{x}\left(Y_{\tau_{U}}^{D} \in \partial U\right)=0
$$

We will use $\zeta$ to denote the lifetime of $Y^{D}$. Then it follows from [26, Corollary 4.2(i)] that the process $Y^{D}$ dies inside $D$ almost surely, i.e.,

$$
\mathbb{P}_{x}\left(Y_{\zeta-}^{D} \in D\right)=1 \quad \text { for all } x \in D
$$

For any open set $U \subset D$, let $Y^{D, U}$ be the subprocess of $Y^{D}$ killed upon exiting $U$ and

$$
r^{D, U}(t, x, y):=r^{D}(t, x, y)-\mathbb{E}_{x}\left[r^{D}\left(t-\tau_{U}, Y_{\tau_{U}}^{D}, y\right): \tau_{U}<t\right] \quad t>0, x, y \in U .
$$

By the strong Markov property, $r^{D, U}(t, x, y)$ is the transition density of $Y^{D, U}$. Then the Green function of $Y^{D, U}$ is given by $G_{U}^{Y^{D}}(x, y):=\int_{0}^{\infty} r^{D, U}(t, x, y) d t$. Further, let $Y_{t}^{U}:=Z^{U}\left(T_{t}\right)$ be the process obtained by subordinating the killed process $Z^{U}$ via the subordinator $T$. The Green function of $Y^{U}$ will be denoted by $G^{Y^{U}}(x, y), x, y \in U$. Since the semigroup of $Y^{U}$ is subordinate to the semigroup of $Y^{D, U}$, cf. [26, Proposition 3.1], we have

$$
G^{Y^{U}}(x, y) \leq G_{U}^{Y^{D}}(x, y) \leq G^{Y^{D}}(x, y) \quad \text { for all } x, y \in U
$$

Note that one can follow the argument in [18, Section 2] and see that $\left(R_{t}^{D}\right)_{t \geq 0}$ satisfies the strong Feller property. 


\section{Comparability of Green functions of $Y^{D}$ and $X$ on small open sets}

In the remainder of the paper, we always assume that $d \geq 2$, (2.1) and (2.2) hold true, and $D$ is a bounded open set in $\mathbb{R}^{d}$.

Definition 3.1 Let $U \subset \mathbb{R}^{d}$ be an open set and let $Q \in \partial U$. We say that $U$ is $C^{1,1}$ near $Q$ if there exist a localization radius $R>0$, a $C^{1,1}$-function $\varphi_{Q}: \mathbb{R}^{d-1} \rightarrow \mathbb{R}$ satisfying $\varphi_{Q}(0)=0$, $\nabla \varphi_{Q}(0)=(0, \ldots, 0),\left\|\nabla \varphi_{Q}\right\|_{\infty} \leq \Lambda,\left|\nabla \varphi_{Q}(z)-\nabla \varphi_{Q}(w)\right| \leq \Lambda|z-w|$, and an orthonormal coordinate system $C S_{Q}$ with its origin at $Q$ such that

$$
B(Q, R) \cap U=\left\{y=\left(\widetilde{y}, y_{d}\right) \in B(0, R) \text { in } C S_{Q}: y_{d}>\varphi_{Q}(\widetilde{y})\right\}
$$

where $\widetilde{y}:=\left(y_{1}, \ldots, y_{d-1}\right)$. The pair $(R, \Lambda)$ is called the $C^{1,1}$ characteristics of $U$ near $Q$. An open set $U \subset \mathbb{R}^{d}$ is said to be a (uniform) $C^{1,1}$ open set with characteristics $(R, \Lambda)$ if it is $C^{1,1}$ with characteristics $(R, \Lambda)$ near every boundary point $Q \in \partial U$.

Recall that, for any open set $U \subset D, X^{U}$ (respectively, $Y^{D, U}$ ) is the process $X$ (respectively, $Y^{D}$ ) killed upon exiting $U$. The approach and proofs in this section are very similar to that of [18, Section 7]; we first show that when $U$ is a relatively compact open subset of $D$, the process $Y^{D, U}$ can be thought of as a non-local Feynman-Kac transform of $X^{U}$. Second, if $U$ is a certain $C^{1,1}$ open set, the Green functions of $X^{U}$ and $Y^{D, U}$ are comparable because the conditional gauge function related to this transform is bounded between two positive constants. We do not give full proofs of the results which are almost identical to the ones in [18, Section 7]. For the convenience of our readers we will provide the exact references for the proofs that we omit.

We denote by $\left(P_{t}\right)_{t \geq 0}$ the semigroup of $Z$. Let $\left(\mathcal{E}^{X^{U}}, \mathcal{D}\left(\mathcal{E}^{X^{U}}\right)\right)$ be the Dirichlet form of $X^{U}$. Then, cf. [22, Section 13.4],

$$
\begin{aligned}
\mathcal{E}^{X^{U}}(f, f) & =\int_{0}^{\infty} \int_{U} f(x)\left(f(x)-P_{s} f(x)\right) d x \nu(s) d s, \\
\mathcal{D}\left(\mathcal{E}^{X^{U}}\right) & =\left\{f \in L^{2}(U, d x): \mathcal{E}^{X^{U}}(f, f)<\infty\right\} .
\end{aligned}
$$

Furthermore, for $f \in \mathcal{D}\left(\mathcal{E}^{X^{U}}\right)$,

$$
\mathcal{E}^{X^{U}}(f, f)=\frac{1}{2} \int_{U} \int_{U}(f(x)-f(y))^{2} J^{X}(x, y) d y d x+\int_{U} f(x)^{2} \kappa_{U}^{X}(x) d x,
$$

where $J^{X}$ is defined in (2.21) and

$$
\kappa_{U}^{X}(x):=\int_{\mathbb{R}^{d} \backslash U} J^{X}(x, y) d y .
$$

Recall that $\left(P_{t}^{D}\right)_{t \geq 0}$ is the semigroup of $Z^{D}$. The Dirichlet form $\left(\mathcal{E}^{Y^{D}}, \mathcal{D}\left(\mathcal{E}^{Y^{D}}\right)\right)$ of $Y^{D}$ is given by

$$
\mathcal{E}^{Y^{D}}(f, f)=\int_{0}^{\infty} \int_{D} f(x)\left(f(x)-P_{s}^{D} f(x)\right) d x \nu(s) d s
$$


and $\mathcal{D}\left(\mathcal{E}^{Y^{D}}\right)=\left\{f \in L^{2}(D, d x): \mathcal{E}^{Y^{D}}(f, f)<\infty\right\}$. Moreover, for $f \in \mathcal{D}\left(\mathcal{E}^{Y^{D}}\right)$,

$$
\mathcal{E}^{Y^{D}}(f, f)=\frac{1}{2} \int_{D} \int_{D}(f(x)-f(y))^{2} J^{Y^{D}}(x, y) d y d x+\int_{D} f(x)^{2} \kappa^{Y^{D}}(x) d x,
$$

where $J^{Y^{D}}$ is defined in (2.20) and

$$
\kappa^{Y^{D}}(x):=\int_{0}^{\infty}\left(1-P_{t}^{D} 1(x)\right) \nu(t) d t .
$$

Hence, it follows that the Dirichlet form $\left(\mathcal{E}^{Y^{D, U}}, \mathcal{D}\left(\mathcal{E}^{Y^{D, U}}\right)\right)$ of $Y^{D, U}$ is given by

$$
\begin{aligned}
\mathcal{E}^{Y^{D, U}}(f, f) & =\int_{0}^{\infty} \int_{U} f(x)\left(f(x)-P_{s}^{D} f(x)\right) d x \nu(s) d s, \\
\mathcal{D}\left(\mathcal{E}^{Y^{D, U}}\right) & =\left\{f \in L^{2}(U, d x): \mathcal{E}^{Y^{D, U}}(f, f)<\infty\right\} .
\end{aligned}
$$

We will need the following simple result. Recall that $\delta_{U}(x)$ is the distance between $x$ and the boundary $\partial U$.

Lemma 3.2 For $x, y \in D$,

$$
J^{X}(x, y)-J^{Y^{D}}(x, y) \leq j^{X}\left(\delta_{D}(y)\right) .
$$

Proof. By (2.14), (2.20) and (2.21), we have that for $x, y \in D$,

$$
\begin{aligned}
J^{X}(x, y)-J^{Y^{D}}(x, y) & =\int_{0}^{\infty} \mathbb{E}_{x}\left[p\left(t-\tau_{D}^{Z}, Z\left(\tau_{D}^{Z}\right), y\right), \tau_{D}^{Z}<t\right] \nu(t) d t \\
& =\mathbb{E}_{x}\left[\int_{\tau_{D}^{Z}}^{\infty} p\left(t-\tau_{D}^{Z}, Z\left(\tau_{D}^{Z}\right), y\right) \nu(t) d t\right]
\end{aligned}
$$

Since, $p(s, z-y) \leq p\left(s, \delta_{D}(y)\right)$ for every $s>0$ and $z \in D^{c}$, we have that for every $s<t$ and $(y, z) \in D \times D^{c}$

$$
\begin{aligned}
\int_{s}^{\infty} p(t-s, z-y) \nu(t) d t & \leq \int_{0}^{\infty} p\left(u, \delta_{D}(y)\right) \nu(u+s) d u \\
& \leq \int_{0}^{\infty} p\left(u, \delta_{D}(y)\right) \nu(u) d u=j^{X}\left(\delta_{D}(y)\right) .
\end{aligned}
$$

This and (3.8) imply the lemma.

Using Lemma 3.2, the proof of the next result is the same as that of [18, Lemma 7.2].

Lemma 3.3 Let $U$ be a relatively compact open subset of $D$. Then $\mathcal{D}\left(\mathcal{E}^{X^{U}}\right)=\mathcal{D}\left(\mathcal{E}^{Y^{D, U}}\right)$.

For $x \in D$, let

$$
q_{U}(x):=\int_{U}\left(J^{X}(x, y)-J^{Y^{D}}(x, y)\right) d y .
$$


For $x, y \in D, x \neq y$, let

$$
F(x, y):=\frac{J^{Y^{D}}(x, y)}{J^{X}(x, y)}-1=\frac{J^{Y^{D}}(x, y)-J^{X}(x, y)}{J^{X}(x, y)},
$$

and $F(x, x):=0$. We also define $F(x, \partial):=0$, where $\partial$ is the cemetery point. Then $-1<F(x, y) \leq 0$. Note that

$$
\int_{U} F(x, y) J^{X}(x, y) d y=-q_{U}(x) .
$$

Using Lemma 3.2 and (2.23), the proof of the next result is very similar to (and even simpler than) that of [18, Lemma 7.3]. So we omit the proof.

Lemma 3.4 There exists $b=b(\phi, d)>2$ such that for all $x_{0} \in D$ and all $r \in(0,1 / b]$ satisfying $B\left(x_{0},(b+1) r\right) \subset D$, it holds that

$$
\sup _{x, y \in B\left(x_{0}, r\right)}|F(x, y)| \leq \frac{1}{2} .
$$

Let $b>2$ be the constant from Lemma 3.4. For $r<1 / b$, let $U \subset D$ be an open set such that $\operatorname{diam}(U) \leq r$ and $\operatorname{dist}(U, \partial D) \geq(b+2) r$. Then there exists a ball $B\left(x_{0}, r\right)$ such that $U \subset B\left(x_{0}, r\right)$ and $B\left(x_{0},(b+1) r\right) \subset D$. Since by Lemma 3.4.

$$
|F(x, y)| \leq 1 / 2 \quad \text { for all } x, y \in U,
$$

using the following non-local multiplicative functional

$$
K_{t}^{U}:=\exp \left(\sum_{0<s \leq t} \log \left(1+F\left(X_{s-}^{U}, X_{s}^{U}\right)\right)\right),
$$

we define

$$
T_{t}^{U} f(x):=\mathbb{E}_{x}\left[K_{t}^{U} f\left(X_{t}^{U}\right)\right] .
$$

By [6, (4.5) and Theorem 4.8] and the proof of [18, Lemma 7.4], $\left(T_{t}^{U}\right)_{t \geq 0}$ is a strongly continuous semigroup on $L^{2}(U, d x)$ with associated quadratic form $\left(\mathcal{E}^{Y^{D, U}}, \mathcal{D}\left(\mathcal{E}^{Y^{D, U}}\right)\right)$.

Let $\mathbb{E}_{x}^{y}$ be the expectation with respect to the conditional probability $\mathbb{P}_{x}^{y}$ defined via Doob's $h$-transform, with $h(\cdot)=G_{U}^{X}(\cdot, y)$, starting from $x \in D$. For $x, y \in U, x \neq y$, we define the conditional gauge function for $K_{t}^{U}$

$$
u^{U}(x, y):=\mathbb{E}_{x}^{y}\left[K_{\tau_{U}^{X}}^{U}\right]
$$

which is less than or equal to 1 because $F \leq 0$. By [1, Lemma 3.9], we have that

$$
G_{U}^{Y^{D}}(x, y)=u^{U}(x, y) G_{U}^{X}(x, y), \quad x, y \in U .
$$

Let $\chi(r)=(\psi \circ \phi)(r)$. For $r>0$, define

$$
\chi^{r}(\lambda):=\frac{\chi\left(\lambda r^{-2}\right)}{\chi\left(r^{-2}\right)}, \quad \lambda>0 .
$$


Then $\chi^{r}$ is a complete Bernstein function with $\chi^{r}(1)=1$ and $\chi^{1}(s)=\chi(s)$. It follows from (2.3) that for each $M>0,\left(\chi^{r}\right)_{r \leq M}$ satisfies the following weak scaling condition at infinity, uniformly in $r \in(0, M]$ : there exists $c=c(M)>1$ such that

$$
c^{-1}\left(\frac{S}{s}\right)^{\gamma_{1} \delta_{1}} \leq \frac{\chi^{r}(S)}{\chi^{r}(s)} \leq c\left(\frac{S}{s}\right)^{\gamma_{2} \delta_{2}}, \quad 1<s \leq S<\infty .
$$

Let $K^{r}=\left(K_{t}^{r}\right)_{t \geq 0}$ be a subordinator with Laplace exponent $\chi^{r}$ independent of the Brownian motion $W$. Let $X^{r}=\left(X_{t}^{r}\right)_{t \geq 0}$ be defined by $X_{t}^{r}:=W_{K_{t}^{r}}$ Then $X^{r}$ is an isotropic Lévy process with characteristic exponent $\chi^{r}\left(|\xi|^{2}\right)=\chi\left(|\xi|^{2} r^{-2}\right) / \chi\left(r^{-2}\right), \xi \in \mathbb{R}^{d}$, which shows that $X^{r}$ is identical in law to the process $\left\{r^{-1} X_{t / \chi\left(r^{-2}\right)}\right\}_{t \geq 0}$.

Let $V \subset \mathbb{R}^{d}$ be a bounded $C^{1,1}$ open set. For $r \in(0,1]$, let $V^{r}:=\{r x: x \in V\}$. Denote by $G_{V}^{X^{r}}$ (respectively $G_{V^{r}}^{X}$ ) the Green function of $V$ with respect to $X^{r}$ (respectively the Green function of $V^{r}$ with respect to $X$ ). Then by scaling,

$$
G_{V^{r}}^{X}(x, y)=r^{-d} \chi\left(r^{-2}\right)^{-1} G_{V}^{X^{r}}(x / r, y / r), \quad x, y \in V^{r} .
$$

For any open set $U \subset \mathbb{R}^{d}$, we let

$$
g_{U}^{r}(x, y):=\left(1 \wedge \frac{\chi^{r}\left(|x-y|^{-2}\right)}{\sqrt{\chi^{r}\left(\delta_{U}(x)^{-2}\right) \chi^{r}\left(\delta_{U}(y)^{-2}\right)}}\right) \frac{1}{|x-y|^{d} \chi^{r}\left(|x-y|^{-2}\right)}, \quad x, y \in U,
$$

and $g_{U}(x, y):=g_{U}^{1}(x, y)$.

Proposition 3.5 Let $V \subset \mathbb{R}^{d}$ be a bounded $C^{1,1}$ open set with characteristics $(R, \Lambda)$ and $\operatorname{diam}(V) \leq 1$. There exists a constant $C=C(R, \Lambda, \phi, \psi, d) \geq 1$ such that for every $r \in(0,1]$,

$$
C^{-1} g_{V^{r}}(x, y) \leq G_{V^{r}}^{X}(x, y) \leq C g_{V^{r}}(x, y), \quad x, y \in V^{r} .
$$

The dependence of $c$ on $\phi$ and $\psi$ is only through the constants in assumptions (2.1) and (2.2).

Proof. The proof is similar to that of [18, Proposition 7.5]. In fact, by [9, Theorem 1.2] and (3.11), there exists a constant $c>0$ such that for all $r \in(0,1]$,

$$
c^{-1} g_{V}^{r}(x, y) \leq G_{V}^{X^{r}}(x, y) \leq c g_{V}^{r}(x, y), \quad x, y \in V .
$$

Since $g_{V}^{r}(x / r, y / r)=r^{d} \phi\left(r^{-2}\right) g_{V^{r}}(x, y)$, the claim of the proposition follows by combining this, (3.12) and (3.14).

Note that the fact that we scale around the origin is irrelevant. For any $z \in \mathbb{R}^{d}$ we could use the scaling $V^{r}:=\{r(x-z)+z ; x \in V\}$ and obtain the same result.

Using Proposition 3.5 and (3.9), the proof of the next result is very similar to (and even simpler than) that of [18, Lemmas 7.6-7.8]. So we omit the proof.

Lemma 3.6 Let $R>0$ and $\Lambda>0$. There exists $C=C(R, \Lambda, \phi, \psi, d) \in(0,1)$ such that for every $r \in(0,1 / b]$ and every $C^{1,1}$ open set $U \subset D$ with characteristics $(r R, \Lambda / r)$ and $\operatorname{diam}(U) \leq r$ satisfying $\operatorname{dist}(U, \partial D) \geq(b+2) r$, we have

$$
C \leq u^{U}(x, y) \leq 1, \quad x, y \in U, x \neq y .
$$


Combining this lemma with (3.10) we arrive at

Proposition 3.7 Let $R>0$ and $\Lambda>0$. There exists $C=C(R, \Lambda, \phi, \psi, d) \in(0,1)$ such that for every $r \in(0,1 / b]$ and every $C^{1,1}$ open set $U \subset D$ with characteristics $(r R, \Lambda / r)$ and $\operatorname{diam}(U) \leq r$ satisfying $\operatorname{dist}(U, \partial D) \geq(b+2) r$, we have

$$
C G_{U}^{X}(x, y) \leq G_{U}^{Y^{D}}(x, y) \leq G_{U}^{X}(x, y), \quad x, y \in U
$$

\section{Behavior of harmonic functions in the interior of $D$}

We first recall the definitions of $\kappa$-fat open sets in $\mathbb{R}^{d}$ and harmonic functions.

Definition 4.1 Let $0<\kappa<1$. We say that an open set $D \subset \mathbb{R}^{d}$ is $\kappa$-fat if there is $R_{1}>0$ such that for all $x \in \bar{D}$ and all $r \in\left(0, R_{1}\right]$, there is a ball $B\left(A_{r}(x), \kappa r\right) \subset D \cap B(x, r)$. The pair $\left(R_{1}, \kappa\right)$ is called the characteristics of the $\kappa$-fat open set $D$.

Definition 4.2 Suppose that $D \subset \mathbb{R}^{d}$ is an open set. (1) A non-negative Borel function $u$ on $D$ is said to be harmonic in an open set $U \subset D$ with respect to $Y^{D}$ if for every open set $B$ whose closure is a compact subset of $U$,

$$
u(x)=\mathbb{E}_{x}\left[u\left(Y_{\tau_{B}}^{D}\right)\right], \quad \text { for every } x \in B .
$$

(2) A non-negative Borel function $u$ on $D$ is said to be regular harmonic in an open set $U \subset D$ with respect to $Y^{D}$ if

$$
u(x)=\mathbb{E}_{x}\left[u\left(Y_{\tau_{U}}^{D}\right)\right], \quad \text { for every } x \in U .
$$

Clearly, a regular harmonic function in $U$ is harmonic in $U$.

The first goal of this section is to prove a scale invariant Harnack inequality for nonnegative functions harmonic with respect to $Y^{D}$ when $D$ is a bounded $\kappa$-fat open set in $\mathbb{R}^{d}$. Then we study the decay rate of non-negative functions in $D$ which are (regular) harmonic near a portion of the boundary, strictly contained in $D$, of an open set $E \subset D$ and vanish locally on $E^{c}$.

Let $j^{Z}$ be the Lévy density of $Z$. Recall that $\Phi(r)=\frac{1}{\phi\left(r^{-2}\right)}$. Analogously to (2.23), it follows from [14, Lemma 3.2] that for any $M>0$ there exists $c(M) \geq 1$ such that

$$
c(M)^{-1} \frac{1}{|x-y|^{d} \Phi(|x-y|)} \leq j^{Z}(|x-y|) \leq c(M) \frac{1}{|x-y|^{d} \Phi(|x-y|)}, \quad|x-y| \leq M
$$

The following result is a consequence of [5, Proposition 3.6] and it is valid for any bounded open set $D$.

Lemma 4.3 Let $D \subset \mathbb{R}^{d}$ be a bounded open set and $a, T$ be positive constants. There exists $C=C(a, T, \phi, \operatorname{diam}(D))>0$ such that

$$
p^{D}(t, x, y) \geq C\left(\Phi^{-1}(t)^{-d} \wedge \frac{t}{|x-y|^{d} \Phi(|x-y|)}\right), \quad t \leq \Phi\left(a\left(\delta_{D}(x) \wedge \delta_{D}(y)\right)\right) \wedge T .
$$


Note that

$$
\Phi^{-1}(t)^{-d} \leq \frac{t}{|x-y|^{d} \Phi(|x-y|)} \quad \text { if and only if } \quad t \geq \Phi(|x-y|) .
$$

Lemma 4.4 Let $D \subset \mathbb{R}^{d}$ be a bounded open set and $\varepsilon_{0} \in(0,1]$ be a constant. There exists a constant $C=C\left(\varepsilon_{0}, \phi, \operatorname{diam}(D)\right) \in(0,1)$ such that for every $x_{0} \in D$ and $r \leq 1 / 2$ satisfying $B\left(x_{0},\left(1+\varepsilon_{0}\right) r\right) \subset D$, we have

$$
C J^{X}(x, y) \leq J^{Y^{D}}(x, y) \leq J^{X}(x, y), \quad x, y \in B\left(x_{0}, r\right) .
$$

Proof. The second inequality in (4.5) is (2.22). So we only need to prove the first inequality. It follows from Lemma 4.3 that there exists $c_{1}=c_{1}\left(\varepsilon_{0}, \phi, \operatorname{diam}(D)\right)>0$ such that for any $t \leq \Phi\left(\left(2 / \varepsilon_{0}\right)\left(\delta_{D}(x) \wedge \delta_{D}(y)\right) \wedge \Phi(\operatorname{diam}(D))\right.$,

$$
p^{D}(t, x, y) \geq c_{1}\left(\Phi^{-1}(t)^{-d} \wedge \frac{t}{|x-y|^{d} \Phi(|x-y|)}\right) .
$$

Thus using the fact that $s \mapsto \nu(s)$ is decreasing, (4.4) and (2.5), we have that for $x, y \in$ $B\left(x_{0}, r\right)$,

$$
\begin{aligned}
& J^{Y^{D}}(x, y) \geq \int_{0}^{\Phi(|x-y|)} p^{D}(t, x, y) \nu(t) d t \geq \nu(\Phi(|x-y|)) \int_{0}^{\Phi(|x-y|)} p^{D}(t, x, y) d t \\
& \geq c_{2} \frac{\psi\left(\Phi(|x-y|)^{-1}\right)}{\Phi(|x-y|)} \int_{0}^{\Phi(|x-y|)} \frac{t}{|x-y|^{d} \Phi(|x-y|)} d t \geq c_{3} \frac{\psi\left(\Phi(|x-y|)^{-1}\right)}{|x-y|^{d}} \\
& \geq c_{4} J^{X}(x, y),
\end{aligned}
$$

where in the last inequality we used (2.23).

In the remainder of this section we assume that $D$ is a bounded $\kappa$-fat open set in $\mathbb{R}^{d}$.

Combining [5, Corollary 1.4] with (4.2) we get that for every $T>0$ there exists $C(T) \geq 1$ such that

$$
\begin{aligned}
& C(T)^{-1} \mathbb{P}_{x}\left(\tau_{D}^{Z}>t\right) \mathbb{P}_{y}\left(\tau_{D}^{Z}>t\right)\left(\Phi^{-1}(t)^{-d} \wedge \frac{t}{|x-y|^{d} \Phi(|x-y|)}\right) \\
& \leq p^{D}(t, x, y) \leq C(T) \mathbb{P}_{x}\left(\tau_{D}^{Z}>t\right) \mathbb{P}_{y}\left(\tau_{D}^{Z}>t\right)\left(\Phi^{-1}(t)^{-d} \wedge \frac{t}{|x-y|^{d} \Phi(|x-y|)}\right)
\end{aligned}
$$

for all $(t, x, y) \in(0, T] \times D \times D$.

Proposition 4.5 Suppose that $D \subset \mathbb{R}^{d}$ is a bounded $\kappa$-fat open set. For every $\varepsilon_{0} \in(0,1]$, there exists a constant $C \geq 1$ such that for all $x_{0} \in D$ and all $r \leq 1$ satisfying $B\left(x_{0},(1+\right.$ $\left.\left.\varepsilon_{0}\right) r\right) \subset D$, it holds that

$$
J^{Y^{D}}\left(z, x_{1}\right) \leq \operatorname{CJ}^{Y^{D}}\left(z, x_{2}\right), \quad x_{1}, x_{2} \in B\left(x_{0}, r\right), \quad z \in D \backslash B\left(x_{0},\left(1+\varepsilon_{0}\right) r\right) .
$$


Proof. We closely follow the proof of [18, Proposition 3.5]. Suppose that $r, \varepsilon_{0} \leq 1, B\left(x_{0},(1+\right.$ $\left.\left.\varepsilon_{0}\right) r\right) \subset D$ and $x_{1}, x_{2} \in B\left(x_{0}, r\right)$. Then it follows from [5, Lemma 3.2] that for $t<\Phi\left(\varepsilon_{0} r\right)$, we have

$$
\mathbb{P}_{x_{2}}\left(t<\tau_{D}^{Z}\right) \geq \mathbb{P}_{x_{2}}\left(t<\tau_{B\left(x_{2}, \Phi^{-1}(t)\right)}^{Z}\right) \geq c_{1}
$$

for some constant $c_{1}=c_{1}\left(\varepsilon_{0}\right)>0$ independent of $x_{2}$ and $t<\Phi\left(\varepsilon_{0} r\right)$. By combining this with (4.6) we have that there exists $c_{2}>1$ such that for $z \in D$ and $t<\Phi\left(\varepsilon_{0} r\right)$,

$$
\begin{aligned}
& p^{D}\left(t, z, x_{1}\right) \leq c_{2} \mathbb{P}_{z}\left(t<\tau_{D}^{Z}\right)\left(\Phi^{-1}(t)^{-d} \wedge \frac{t}{\left|x_{1}-z\right|^{d} \Phi\left(\left|x_{1}-z\right|\right)}\right) \\
& p^{D}\left(t, z, x_{2}\right) \geq c_{2}^{-1} \mathbb{P}_{z}\left(t<\tau_{D}^{Z}\right)\left(\Phi^{-1}(t)^{-d} \wedge \frac{t}{\left|x_{2}-z\right|^{d} \Phi\left(\left|x_{2}-z\right|\right)}\right) .
\end{aligned}
$$

Now suppose that $z \in D \backslash B\left(x_{0},\left(1+\varepsilon_{0}\right) r\right)$ so that

$$
\frac{\varepsilon_{0}}{1+\varepsilon_{0}}\left|z-x_{0}\right| \leq\left|z-x_{i}\right| \leq\left(1+\frac{1}{1+\varepsilon_{0}}\right)\left|z-x_{0}\right|, \quad i=1,2
$$

Then

$$
\begin{aligned}
& \int_{0}^{\Phi\left(\varepsilon_{0} r\right)} p^{D}\left(t, z, x_{1}\right) \nu(t) d t \\
& \leq c_{3} \int_{0}^{\Phi\left(\varepsilon_{0} r\right)} \mathbb{P}_{z}\left(t<\tau_{D}^{Z}\right)\left(\Phi^{-1}(t)^{-d} \wedge \frac{t}{\left|z-x_{2}\right|^{d} \Phi\left(\left|z-x_{2}\right|\right)}\right) \nu(t) d t \\
& \leq c_{3} c_{2} \int_{0}^{\Phi\left(\varepsilon_{0} r\right)} p^{D}\left(t, z, x_{2}\right) \nu(t) d t .
\end{aligned}
$$

Using the parabolic Harnack principle (see, for instance, [2, Theorem 1.4]), we get that there exists $c_{4}>1$ such that

$$
\begin{aligned}
& \int_{\Phi\left(\varepsilon_{0} r\right)}^{\infty} p^{D}\left(t, z, x_{1}\right) \nu(t) d t=\sum_{n=1}^{\infty} \int_{n \Phi\left(\varepsilon_{0} r\right)}^{(n+1) \Phi\left(\varepsilon_{0} r\right)} p^{D}\left(t, z, x_{1}\right) \nu(t) d t \\
& \leq c_{4} \sum_{n=1}^{\infty} \int_{n \Phi\left(\varepsilon_{0} r\right)}^{(n+1) \Phi\left(\varepsilon_{0} r\right)} p^{D}\left(t+\frac{\Phi\left(\varepsilon_{0} r\right)}{2}, z, x_{2}\right) \nu(t) d t \\
& =c_{4} \sum_{n=1}^{\infty} \int_{\left(n+\frac{1}{2}\right) \Phi\left(\varepsilon_{0} r\right)}^{\left(n+\frac{3}{2}\right) \Phi\left(\varepsilon_{0} r\right)} p^{D}\left(t, z, x_{2}\right) \nu\left(t-\frac{\Phi\left(\varepsilon_{0} r\right)}{2}\right) d t .
\end{aligned}
$$

If $t \in\left(\Phi\left(\varepsilon_{0} r\right), 1\right)$, then by (2.6) we have $\nu\left(t-\Phi\left(\varepsilon_{0} r\right) / 2\right) \leq \nu(t / 2) \leq c_{5} \nu(t)$ with $c_{5} \geq 1$. If $t \geq 1$, then $\nu\left(t-\Phi\left(\varepsilon_{0} r\right) / 2\right) \leq \nu(t-1 / 2)$. By (2.7), there exists $c_{6} \geq 1$ such that $\nu(s) \leq c_{6} \nu(s+1 / 2)$ for all $s>1 / 2$. Hence, $\nu(t-1 / 2) \leq c_{6} \nu(t)$. With $c_{7}=c_{5} \vee c_{6}$, we conclude that $\nu\left(t-\Phi\left(\varepsilon_{0} r\right) / 2\right) \leq c_{7} \nu(t)$ for all $t \geq 3 \Phi\left(\varepsilon_{0} r\right) / 2$. Hence,

$$
\int_{\Phi\left(\varepsilon_{0} r\right)}^{\infty} p^{D}\left(t, z, x_{1}\right) \nu(t) d t \leq c_{4} c_{7} \sum_{n=1}^{\infty} \int_{\left(n+\frac{1}{2}\right) \Phi\left(\varepsilon_{0} r\right)}^{\left(n+\frac{3}{2}\right) \Phi\left(\varepsilon_{0} r\right)} p^{D}\left(t, z, x_{2}\right) \nu(t) d t
$$




$$
\leq c_{8} \int_{\left(3 \Phi\left(\varepsilon_{0} r\right)\right) / 2}^{\infty} p^{D}\left(t, z, x_{2}\right) \nu(t) d t .
$$

Combining (2.20), (4.8) and (4.9), we get that there exists $c_{9}>1$ such that

$$
J^{Y^{D}}\left(z, x_{1}\right)=\int_{0}^{\infty} p^{D}\left(t, z, x_{1}\right) \nu(t) d t \leq c_{9} \int_{0}^{\infty} p^{D}\left(t, z, x_{2}\right) \nu(t) d t=c_{9} J^{Y^{D}}\left(z, x_{2}\right),
$$

which finishes the proof.

Recall that $b \geq 2$ is the constant in Lemma 3.4 .

Lemma 4.6 Suppose that $D \subset \mathbb{R}^{d}$ is a bounded $\kappa$-fat open set. There exists $C>0$ such that for every $x_{0} \in D$ and every $r \in(0,1)$ with $B\left(x_{0}, r\right) \subset D$,

$$
\mathbb{E}_{x} \tau_{B\left(x_{0}, r\right)} \geq \mathbb{E}_{x} \tau_{B\left(x_{0}, 4 a r\right)} \geq C \frac{1}{\psi\left(\Phi(r)^{-1}\right)}, \quad x \in B\left(x_{0}, a r\right),
$$

where $a:=2^{-5} b^{-1} \in\left(0,2^{-6}\right]$.

Proof. The lemma follows from (2.3), Propositions 3.5 and 3.7. In fact, by Propositions 3.5 and 3.7. we have that for $x, y \in B\left(x_{0}, 2 a r\right)$,

$$
G_{B\left(x_{0}, 4 a r\right)}^{Y^{D}}(x, y) \geq c_{1} G_{B\left(x_{0}, 4 a r\right)}^{X}(x, y) \geq c_{2} \frac{1}{|x-y|^{d} \psi\left(\Phi(|x-y|)^{-1}\right)} .
$$

Thus for $x \in B\left(x_{0}, a r\right)$,

$$
\begin{aligned}
& \mathbb{E}_{x} \tau_{B\left(x_{0}, 4 a r\right)}=\int_{B\left(x_{0}, 4 a r\right)} G_{B\left(x_{0}, 4 a r\right)}^{Y^{D}}(x, y) d y \geq c_{2} \int_{B\left(x_{0}, 2 a r\right)} \frac{1}{|x-y|^{d} \psi\left(\Phi(|x-y|)^{-1}\right)} d y \\
& \geq c_{2} \int_{B(x, a r)} \frac{1}{|x-y|^{d} \psi\left(\Phi(|x-y|)^{-1}\right)} d y \geq c_{3} \int_{0}^{a r} \psi\left(\Phi(s)^{-1}\right) s^{-1} d s .
\end{aligned}
$$

Since (2.3) implies that

$$
\begin{aligned}
& \int_{0}^{a r} \frac{d s}{\psi\left(\Phi(s)^{-1}\right) s}=\frac{1}{\psi\left(\Phi(a r)^{-1}\right)} \int_{0}^{a r} \frac{\psi\left(\Phi(a r)^{-1}\right)}{\psi\left(\Phi(s)^{-1}\right) s} d s \\
& \geq c_{4} \frac{1}{\psi\left(\Phi(r)^{-1}\right)} \int_{0}^{a r}\left(\frac{s}{a r}\right)^{2 \gamma_{2} \delta_{2}} s^{-1} d s \geq c_{5} \frac{1}{\psi\left(\Phi(r)^{-1}\right)}
\end{aligned}
$$

we have proved the lemma.

By using (2.19), (2.15) and (2.3), one gets that there exists $c>0$ such that for every $x_{0} \in D$ and every $r \in(0,1)$ with $B\left(x_{0}, r\right) \subset D$, we have

$$
\mathbb{E}_{x} \tau_{B\left(x_{0}, r\right)} \leq c \frac{1}{\psi\left(\Phi(r)^{-1}\right)}, \quad x \in B\left(x_{0}, r\right) .
$$

For any open set $U \subset D$, let

$$
K^{D, U}(x, z):=\int_{U} G_{U}^{Y^{D}}(x, y) J^{Y^{D}}(y, z) d y, \quad x \in U, z \in \bar{U}^{c} \cap D
$$

be the Poisson kernel of $Y^{D}$ on $U$. 
Theorem 4.7 (Harnack inequality) Suppose that $D \subset \mathbb{R}^{d}$ is a bounded $\kappa$-fat open set. There exists a constant $C>0$ such that for any $r \in(0,1]$ and $B\left(x_{0}, r\right) \subset D$ and any Borel function $f$ which is non-negative in $D$ and harmonic in $B\left(x_{0}, r\right)$ with respect to $Y^{D}$, we have

$$
f(x) \leq C f(y), \quad \text { for all } x, y \in B\left(x_{0}, r / 2\right) .
$$

Proof. Let $b_{1}=a / 2$ and $b_{2}=a / 4$, where $a$ is the constant from Lemma 4.6. We will first show that there exists a constant $c_{1}>0$, independent of $x_{0}$ and $r$, such that for all $y_{0} \in B\left(x_{0}, r / 2\right), x_{1}, x_{2} \in B\left(y_{0}, b_{2} r / 4\right)$ and $z \in{\overline{B\left(y_{0}, b_{1} r\right.}}^{c} \cap D$,

$$
K^{D, B\left(y_{0}, b_{1} r\right)}\left(x_{1}, z\right) \leq c_{1} K^{D, B\left(y_{0}, b_{1} r\right)}\left(x_{2}, z\right) .
$$

Let $A\left(x, r_{1}, r_{2}\right):=\left\{y \in \mathbb{R}^{d}: r_{1} \leq|y-x|<r_{2}\right\}$. Note that

$$
\begin{aligned}
& K^{D, B\left(y_{0}, b_{1} r\right)}\left(x_{1}, z\right) \\
& =\int_{B\left(y_{0}, b_{2} r\right)} G_{B\left(y_{0}, b_{1} r\right)}^{Y^{D}}\left(x_{1}, y\right) J^{Y^{D}}(y, z) d y+\int_{A\left(y_{0}, b_{2} r, b_{1} r\right)} G_{B\left(y_{0}, b_{1} r\right)}^{Y^{D}}\left(x_{1}, y\right) J^{Y^{D}}(y, z) d y \\
& =: I_{1}+I_{2} .
\end{aligned}
$$

In order to estimate $I_{2}$ we use Propositions 3.5 and 3.7 together with $\left|x_{1}-y\right| \asymp^{c_{2}}\left|x_{2}-y\right|$ for all $y \in A\left(y_{0}, b_{2} r, b_{1} r\right)$ and $\delta_{B\left(y_{0}, b_{1} r\right)}\left(x_{1}\right) \asymp^{c_{3}} \delta_{B\left(y_{0}, b_{1} r\right)}\left(x_{2}\right)$ to get

$$
I_{2} \leq c_{4} \int_{A\left(y_{0}, b_{2} r, b_{1} r\right)} G_{B\left(y_{0}, b_{1} r\right)}^{Y^{D}}\left(x_{2}, y\right) J^{Y^{D}}(y, z) d y \leq c_{5} K^{D, B\left(y_{0}, b_{1} r\right)}\left(x_{2}, z\right) .
$$

By Proposition 4.5 (with $\left.\varepsilon_{0}=1\right), J^{Y^{D}}\left(y_{0}, z\right) \asymp^{c_{6}} J^{Y^{D}}(y, z)$ for $z \in B\left(y_{0}, b_{1} r\right)^{c}=B\left(y_{0}, 2 b_{2} r\right)^{c}$ and $y \in B\left(y_{0}, b_{2} r\right)$. Hence, by (4.10) and Lemma 4.6,

$$
\begin{aligned}
I_{1} & \leq c_{7} J^{Y^{D}}\left(y_{0}, z\right) \int_{B\left(y_{0}, b_{2} r\right)} G_{B\left(y_{0}, b_{1} r\right)}^{Y^{D}}\left(x_{1}, y\right) d y \leq c_{7} J^{Y^{D}}\left(y_{0}, z\right) \mathbb{E}_{x_{1}} \tau_{B\left(y_{0}, b_{1} r\right)} \\
& \leq c_{8} J^{Y^{D}}\left(y_{0}, z\right) \psi\left(\Phi(r)^{-1}\right)^{-1} \leq c_{9} J^{Y^{D}}\left(y_{0}, z\right) \mathbb{E}_{x_{2}} \tau_{B\left(y_{0}, b_{2} r\right)} \\
& =c_{9} J^{Y^{D}}\left(y_{0}, z\right) \int_{B\left(y_{0}, b_{2} r\right)} G_{B\left(y_{0}, b_{2} r\right)}^{Y^{D}}\left(x_{2}, y\right) d y \\
& \leq c_{9} J^{Y^{D}}\left(y_{0}, z\right) \int_{B\left(y_{0}, b_{2} r\right)} G_{B\left(y_{0}, b_{1} r\right)}^{Y^{D}}\left(x_{2}, y\right) d y \\
& \leq c_{10} \int_{B\left(y_{0}, b_{2} r\right)} G_{B\left(y_{0}, b_{1} r\right)}^{Y^{D}}\left(x_{2}, y\right) J^{Y^{D}}(y, z) d y \leq c_{10} K^{D, B\left(y_{0}, b_{1} r\right)}\left(x_{2}, z\right) .
\end{aligned}
$$

Combining (4.12)-(4.14), we have proved (4.11). Now, let $f$ be a non-negative function in $D$ which is harmonic with respect to $Y^{D}$ in $B\left(x_{0}, r\right)$. Then, by the Lévy system formula and (2.24), for all $y_{0} \in B\left(x_{0}, r / 2\right)$ and $x_{1}, x_{2} \in B\left(y_{0}, b_{1} r / 8\right)$ we have

$$
\begin{aligned}
& f\left(x_{1}\right)=\int_{{\overline{B\left(y_{0}, b_{1} r\right)^{c}}}^{c} K^{D, B\left(y_{0}, b_{1} r\right)}\left(x_{1}, z\right) f(z) d z} \leq c_{11} \int_{{\overline{B\left(y_{0}, b_{1} r\right)}}^{c}} K^{D, B\left(y_{0}, b_{1} r\right)}\left(x_{2}, z\right) f(z) d z=c_{11} f\left(x_{2}\right) .
\end{aligned}
$$

For $x_{1}, x_{2} \in B\left(x_{0}, r / 2\right)$, the theorem follows by a standard chain argument.

Now we prove the following version of the Harnack inequality. 
Theorem 4.8 Suppose that $D \subset \mathbb{R}^{d}$ is a bounded $\kappa$-fat open set. There exists a constant $C=C(\phi, \psi, \operatorname{diam}(D))>1$ such that the following is true: If $L>0$ and $x_{1}, x_{2} \in D$ and $r \in(0,1)$ are such that $\left|x_{1}-x_{2}\right|<\operatorname{Lr}$ and $B\left(x_{1}, r\right) \cup B\left(x_{2}, r\right) \subset D$, then for any Borel function $f$ which is non-negative in $D$ and harmonic in $B\left(x_{1}, r\right) \cup B\left(x_{2}, r\right)$ with respect to $Y^{D}$, we have

$$
C^{-1}(L \vee 1)^{-d-\delta_{2}} f\left(x_{2}\right) \leq f\left(x_{1}\right) \leq C(L \vee 1)^{d+\delta_{2}} f\left(x_{2}\right)
$$

Proof. Let $r \in(0,1), x_{1}, x_{2} \in D$ be such that $\left|x_{1}-x_{2}\right|<\operatorname{Lr}$ and $B\left(x_{1}, r\right) \cup B\left(x_{2}, r\right) \subset D$. Let $f$ be a non-negative function which is harmonic in $B\left(x_{1}, r\right) \cup B\left(x_{2}, r\right)$ with respect to $Y^{D}$. If $\left|x_{1}-x_{2}\right|<\frac{1}{4} r$, then since $r<1$, the claim is true by Theorem 4.7. Thus we only need to consider the case when $\frac{1}{4} r \leq\left|x_{1}-x_{2}\right| \leq L r$ with $L>\frac{1}{4}$.

By Lemma 4.3, (2.1) and (2.5), for every $(x, y) \in B\left(x_{2}, \frac{r}{16}\right) \times B\left(x_{1}, \frac{r}{16}\right)$,

$$
\begin{aligned}
& J^{Y^{D}}(x, y) \geq \int_{0}^{\Phi\left(\delta_{D}(x) \wedge \delta_{D}(y)\right)} p^{D}(t, x, y) \nu(t) d t \\
& \geq c_{1} \int_{0}^{\Phi\left(\delta_{D}(x) \wedge \delta_{D}(y)\right)}\left(\Phi^{-1}(t)^{-d} \wedge \frac{t}{|x-y|^{d} \Phi(|x-y|)}\right) \nu(t) d t \\
& \geq c_{2} \int_{0}^{\Phi(r / 8)} \frac{t \nu(t)}{|x-y|^{d} \Phi(|x-y|)} d t \geq c_{3} \nu(\Phi(r / 8)) \int_{0}^{\Phi(r / 8)} \frac{t}{|x-y|^{d} \Phi(|x-y|)} d t \\
& \geq c_{4} \nu(\Phi(r / 8)) \int_{0}^{\Phi(r / 8)} \frac{t}{(2 L r)^{d} \Phi(2 L r)} d t \geq c_{5} \psi\left(\Phi(r / 8)^{-1}\right) \frac{\Phi(r / 8)}{(2 L r)^{d} \Phi(L r)} \\
& \geq c_{6} L^{-d-\delta_{2}} r^{-d} \psi\left(\Phi(r / 8)^{-1}\right) .
\end{aligned}
$$

Note that, by Proposition 4.5, for every $y \in B\left(x_{1}, \frac{r}{16}\right)$, it holds that

$$
K^{D, B\left(x_{2}, \frac{r}{16}\right)}\left(x_{2}, y\right)=\int_{B\left(x_{2}, \frac{r}{16}\right)} G_{B\left(x_{2}, \frac{r}{16}\right)}^{Y^{D}}\left(x_{2}, z\right) J^{Y^{D}}(z, y) d z \geq c_{7} J^{Y^{D}}\left(x_{2}, y\right) \mathbb{E}_{x_{2}}\left[\tau_{B\left(x_{2}, \frac{r}{16}\right)}\right] .
$$

Thus using this, (4.15) and Lemma 4.6. we have that for every $y \in B\left(x_{1}, \frac{r}{16}\right)$,

$$
K^{D, B\left(x_{2}, \frac{r}{16}\right)}\left(x_{2}, y\right) \geq c_{7} c_{6} L^{-d-\delta_{2}} r^{-d} \frac{\psi\left(\Phi(r / 8)^{-1}\right)}{\psi\left(\Phi(r /(16))^{-1}\right)} \geq c_{8} L^{-d-\delta_{2}} r^{-d} .
$$

For any $y \in B\left(x_{1}, \frac{r}{16}\right), f$ is regular harmonic in $B\left(y, \frac{15 r}{16}\right) \cup B\left(x_{1}, \frac{15 r}{16}\right)$. Since $\left|y-x_{1}\right|<\frac{r}{16}$, by Theorem 4.7 .

$$
f(y) \geq c_{9} f\left(x_{1}\right), \quad y \in B\left(x_{1}, \frac{r}{16}\right),
$$

for some constant $c_{9}>0$. Therefore, by (4.16),

$$
\begin{aligned}
f\left(x_{2}\right) & =\mathbb{E}_{x_{2}}\left[f\left(Y_{\left.\tau_{B\left(x_{2}, \frac{r}{16}\right.}\right)}^{D}\right)\right] \geq \mathbb{E}_{x_{2}}\left[f\left(Y_{\tau_{B\left(x_{2}, \frac{r}{16}\right)}^{D}}^{D}\right) ; Y_{\tau_{B\left(x_{2}, \frac{r}{16}\right)}^{D}}^{D} \in B\left(x_{1}, \frac{r}{16}\right)\right] \\
& \geq c_{10} f\left(x_{1}\right) \mathbb{P}_{x_{2}}\left(Y_{\tau_{B\left(x_{2}, \frac{r}{16}\right)}^{D}}^{D} \in B\left(x_{1}, \frac{r}{16}\right)\right)=c_{10} f\left(x_{1}\right) \int_{B\left(x_{1}, \frac{r}{16}\right)} K^{D, B\left(x_{2}, \frac{r}{16}\right)}\left(x_{2}, w\right) d w \\
& \geq c_{11} L^{-d-\delta_{2}} f\left(x_{1}\right)\left|B\left(x_{1}, \frac{r}{16}\right)\right| r^{-d}=c_{12} L^{-d-\delta_{2}} f\left(x_{1}\right) .
\end{aligned}
$$

Thus we have proved the theorem. 
Remark 4.9 Suppose that $d=1$ and that $D$ is a bounded open set which is a union of disjoint open intervals so that the infimum of the lengths of all these intervals is at least $R_{0}$ and the infimum of the distances between these intervals is at least $R_{0}$.

Let $\widetilde{D}=D \times[0,1]$ and let $\widetilde{Y} \widetilde{D}$ be the subordinate killed Brownian motion in $\widetilde{D} \subset \mathbb{R}^{2}$. By checking the definitions, we can see that if $h$ is harmonic in $U \subset D$ with respect to $Y^{D}$, then $\widetilde{h}(x, y)=h(x), x \in D, y \in[0,1]$, is harmonic in $U \times[0,1]^{2}$ with respect to $\widetilde{Y}^{\widetilde{D}}$. Note that $U \times[0,1]$ is a $\kappa$-fat open set in $\mathbb{R}^{2}$. Thus, in fact, using Theorems 4.7 and 4.8 for $d=2$, Theorems 4.7 and 4.8 also hold for $d=1$.

Theorem 4.10 Suppose $d \geq 2$. Let $D \subset \mathbb{R}^{d}$ be a bounded $\kappa$-fat open set. There exists a constant $b=b(\phi, \psi, d)>2$ such that, for every open set $E \subset D$ and every $Q \in \partial E \cap D$ such that $E$ is $C^{1,1}$ near $Q$ with characteristics $(R, \Lambda)$, the following holds: There exists a constant $C=C\left(\delta_{D}(Q) \wedge R, \Lambda, \psi, \phi, d\right)>0$ such that for every $r \leq\left(\delta_{D}(Q) \wedge 1\right) /(b+2)$ and every non-negative function $f$ on $D$ which is regular harmonic in $E \cap B(Q, r)$ with respect to $Y^{D}$ and vanishes on $E^{c} \cap B(Q, r)$, we have

$$
\sqrt{(\psi \circ \phi)\left(\delta_{E}(x)^{-2}\right)} f(x) \leq C \sqrt{(\psi \circ \phi)\left(\delta_{E}(y)^{-2}\right)} f(y), \quad x, y \in E \cap B\left(Q, 2^{-6} \kappa_{0}^{4} r\right),
$$

where $\kappa_{0}=\left(1+(1+\Lambda)^{2}\right)^{-1 / 2}$.

Proof. Using (2.3), [23, Lemma 2.2], Proposition 3.7, Proposition 4.5 and the factorization from either [12, Lemma 5.5] or [9, Lemma 5.4], the proof is the same as that of [18, Theorem 1.3] with $\psi \circ \phi$ instead of $\phi$. So we omit the details.

\section{Carleson estimate}

We will establish the Carleson estimate for $Y^{D}$. Unlike [18], neither the explicit boundary behavior of the jumping kernel nor that of the Green function is used in the proof of the Carleson estimate. The Carleson estimate of $Y^{D}$ is established for a large class of non-smooth open sets.

Using (2.25), the proof of the next lemma is the same as that of [18, Lemma 5.1].

Lemma 5.1 Suppose that $D \subset \mathbb{R}^{d}$ is an open set. Let $x_{0} \in \mathbb{R}^{d}$, and $r_{1}<r_{2}$ be two positive numbers such that $D \cap B\left(x_{0}, r_{1}\right) \neq \emptyset$. Suppose $f$ is a non-negative function in $D$ that is harmonic in $D \cap B\left(x_{0}, r_{2}\right)$ with respect to $Y^{D}$ and vanishes continuously on $\partial D \cap B\left(x_{0}, r_{2}\right)$. Then $f$ is regular harmonic in $D \cap B\left(x_{0}, r_{1}\right)$ with respect to $Y^{D}$, i.e.,

$$
f(x)=\mathbb{E}_{x}\left[f\left(Y^{D}\left(\tau_{D \cap B\left(x_{0}, r_{1}\right)}\right)\right)\right] \quad \text { for all } x \in D \cap B\left(x_{0}, r_{1}\right) .
$$

For $x \in D$, let $z_{x}$ be a point on $\partial D$ such that $\left|z_{x}-x\right|=\delta_{D}(x)$. We say $D \subset \mathbb{R}^{d}$ satisfies the local exterior volume condition with characteristics $\left(R_{0}, C_{0}\right)$ if for every $z \in \partial D$ and $x \in B\left(z, R_{0}\right) \cap D,\left|D^{c} \cap B\left(z_{x}, \delta_{D}(x)\right)\right| \geq C_{0} \delta_{D}(x)^{d}$. It is easy to see that, if $D^{c}$ is $\kappa$-fat, then $D$ satisfies the local exterior volume condition.

We recall that $\zeta$ is the lifetime of $Y^{D}$. Let

$$
g(r):=\frac{1}{r^{d} \psi\left(\Phi(r)^{-1}\right)}, \quad r>0 .
$$


Lemma 5.2 Suppose that $D \subset \mathbb{R}^{d}$ satisfies the local exterior volume condition with characteristics $\left(R_{0}, C_{0}\right)$. Then there exists a constant $\delta_{*}=\delta_{*}\left(R_{0}, C_{0}\right)>0$ such that for all $x \in D$ with $\delta_{D}(x)<R_{0} / 2$,

$$
\mathbb{P}_{x}(\tau(x)=\zeta) \geq \delta_{*},
$$

where $\tau(x):=\tau_{B\left(x, \delta_{D}(x) / 2\right)}=\inf \left\{t>0: Y_{t}^{D} \notin B\left(x, \delta_{D}(x) / 2\right)\right\}$.

Proof. By [7, Theorem 4.5.4(1)],

$$
\mathbb{P}_{x}(\tau(x)=\zeta)=\mathbb{P}_{x}\left(Y_{\zeta-}^{D} \in B\left(x, \delta_{D}(x) / 2\right)\right)=\int_{B\left(x, \delta_{D}(x) / 2\right)} G^{Y^{D}}(x, y) \kappa^{Y^{D}}(y) d y,
$$

where $\kappa^{Y^{D}}$ is the density of the killing measure of $Y^{D}$ given in (3.4). Since $D$ satisfies the local exterior volume condition, we have (see the proof of [16, Proposition 5.12])

$$
\kappa^{Y^{D}}(y) \geq \kappa^{X^{D}}(y) \geq c_{1} \psi\left(\Phi\left(\delta_{D}(y)\right)^{-1}\right), \quad y \in B\left(x, \delta_{D}(x) / 2\right) .
$$

Here (2.4) is used in the first inequality. Thus, using (2.3), (5.3), (2.27), and Propositions 3.5 and 3.7 ,

$$
\begin{aligned}
& \mathbb{P}_{x}(\tau(x)=\zeta)=\int_{B\left(x, \delta_{D}(x) / 2\right)} G^{Y^{D}}(x, y) \kappa^{Y^{D}}(y) d y \\
& \geq \int_{B\left(x, \delta_{D}(x) /(4 b)\right)} G_{B\left(x, \delta_{D}(x) /(8 b)\right)}^{Y^{D}}(x, y) \kappa^{Y^{D}}(y) d y \\
& \geq c_{2} \int_{B\left(x, \delta_{D}(x) /(4 b)\right)} g(|x-y|) \psi\left(\Phi\left(\delta_{D}(y)\right)^{-1}\right) d y \\
& \geq c_{3} \psi\left(\Phi\left(\delta_{D}(x)\right)^{-1}\right) \int_{B\left(x, \delta_{D}(x) /(4 b)\right)} \frac{1}{|x-y|^{d} \psi\left(\Phi(|x-y|)^{-1}\right)} d y \\
& \geq c_{4} \psi\left(\Phi\left(\delta_{D}(x)\right)^{-1}\right) \frac{1}{\psi\left(\Phi\left(\delta_{D}(x) /(4 b)\right)^{-1}\right)} \geq c_{5},
\end{aligned}
$$

where $b>2$ is the constant in Lemma 3.4.

In the remainder of this section we will assume $D \subset \mathbb{R}^{d}$ is a bounded $\kappa$-fat open set with characteristics $\left(R_{1}, \kappa\right)$. Combining (2.20) and [5, Theorem 1.3(iii) and Corollary 1.4], we immediately get the following

Proposition 5.3 For any $T>0$, there exists $C=C\left(R_{1}, \kappa, T\right) \geq 1$ such that for all $x, y \in$ $D$

$$
C^{-1} \widetilde{J}^{D}(x, y) \leq J^{Y^{D}}(x, y) \leq C \widetilde{J}^{D}(x, y)
$$

where

$$
\begin{aligned}
\widetilde{J}^{D}(x, y)=\int_{0}^{T} & \mathbb{P}_{x}\left(\tau_{D}^{Z}>t\right) \mathbb{P}_{y}\left(\tau_{D}^{Z}>t\right)\left(\Phi^{-1}(t)^{-d} \wedge \frac{t}{|x-y|^{d} \Phi(|x-y|)}\right) \nu(t) d t \\
& +\mathbb{P}_{x}\left(\tau_{D}^{Z}>1\right) \mathbb{P}_{y}\left(\tau_{D}^{Z}>1\right) .
\end{aligned}
$$

Before we prove the Carleson estimate for $Y^{D}$, we first show the following form of parabolic Carleson type estimate for $Z$. 
Proposition 5.4 For any $T>0$ and $c_{0} \in(0,1)$, there exists $C=C\left(R_{1}, \kappa, c_{0}, T\right) \geq 1$ such that for all $t \in(0, T], r \leq R_{1} / 2, Q \in \partial D$ and $x, x_{0} \in D \cap B(Q, r)$ with $\delta_{D}\left(x_{0}\right) \geq c_{0} r$,

$$
\mathbb{P}_{x}\left(\tau_{D}^{Z}>t\right) \leq C \mathbb{P}_{x_{0}}\left(\tau_{D}^{Z}>t\right)
$$

Proof. For simplicity, without loss of generality we assume $T=R_{1}=1$. In this proof, we always assume that $t, r \in(0,1]$ and $x, x_{0} \in D \cap B(Q, r)$ with $\delta_{D}\left(x_{0}\right) \geq c_{0} r$.

Case 1. $r \geq 2^{-4} \kappa \Phi^{-1}(t) / 3$ : In this case by [5, Lemma 3.2] and (2.9),

$$
\begin{aligned}
& \mathbb{P}_{x_{0}}\left(\tau_{D}^{Z}>t\right) \geq \mathbb{P}_{x_{0}}\left(\tau_{B\left(x_{0}, c_{0} r\right)}^{Z}>t\right) \geq \mathbb{P}_{x_{0}}\left(\tau_{B\left(x_{0}, c_{0} r\right)}^{Z}>\Phi\left(3 \cdot 2^{4} \kappa^{-1} r\right)\right) \\
& \geq \mathbb{P}_{x_{0}}\left(\tau_{B\left(x_{0}, c_{0} r\right)}^{Z}>c_{2} \Phi\left(c_{0} r\right)\right) \geq c_{3}>0 .
\end{aligned}
$$

Thus $\mathbb{P}_{x}\left(\tau_{D}^{Z}>t\right) \leq 1 \leq c_{3}^{-1} \mathbb{P}_{x_{0}}\left(\tau_{D}^{Z}>t\right)$.

Case 2. $r \leq 2^{-4} \kappa \Phi^{-1}(t) / 3$ : In this case, we will use [5, Lemma 4.1]. Let $A:=A_{\Phi^{-1}(t) / 2}(Q)$ so that $B\left(A, \kappa \Phi^{-1}(t) / 2\right) \subset B\left(Q, \Phi^{-1}(t) / 2\right) \cap D$. Since $B\left(Q, \Phi^{-1}(t) / 2\right) \subset B\left(y, \Phi^{-1}(t)\right)$ for all $y \in B(Q, r)$, we have

$$
B\left(A, 2^{-2} \kappa \Phi^{-1}(t)\right) \subset B\left(A, 2^{-1} \kappa \Phi^{-1}(t)\right) \subset B\left(y, \Phi^{-1}(t)\right) \cap D, \quad \forall y \in B(Q, r) \cap D .
$$

Define

$$
\begin{array}{r}
U(x):=D \cap B\left(x,|x-A|+2^{-2} \kappa \Phi^{-1}(t) / 3\right), \\
\widehat{U}\left(x_{0}\right):=D \cap B\left(x_{0},\left|x_{0}-A\right|+2^{-1} \kappa \Phi^{-1}(t) / 3\right) .
\end{array}
$$

Note that $U(x) \subset \widehat{U}\left(x_{0}\right)$. In fact, by assumption, we have $\left|x-x_{0}\right| \leq 2^{-3} \kappa \Phi^{-1}(t) / 3$, so for $y \in U(x)$

$$
\begin{aligned}
\left|x_{0}-y\right| & \leq\left|x-x_{0}\right|+|x-A|+2^{-2} \kappa \Phi^{-1}(t) / 3 \\
& \leq\left|x_{0}-A\right|+2\left|x-x_{0}\right|+2^{-2} \kappa \Phi^{-1}(t) / 3 \\
& \leq\left|x_{0}-A\right|+2^{-1} \kappa \Phi^{-1}(t) / 3 .
\end{aligned}
$$

Since $D$ is $2^{-2} \kappa$-fat and $A=A_{2^{-2} \kappa \Phi^{-1}(t)}(x)$ by (5.5), using [5, Lemma 4.1], we have

$$
\mathbb{P}_{x}\left(\tau_{D}^{Z}>t\right) \leq c_{4} \mathbb{P}_{x}\left(Z_{\tau_{U(x)}} \in D\right)
$$

Since $y \rightarrow \mathbb{P}_{y}\left(Z_{\tau_{U(x)}} \in D\right)$ is regular harmonic in $D \cap B(Q, 2 r)$ with respect to $Z$ and vanishes in $D^{c} \cap B(Q, 2 r)$, by [12, Lemma 5.5],

$$
\mathbb{P}_{x}\left(Z_{\tau_{U(x)}} \in D\right) \leq c_{5} \mathbb{P}_{x_{0}}\left(Z_{\tau_{U(x)}} \in D\right) .
$$

Since $x_{0} \in B\left(x, 2^{-3} \kappa \Phi^{-1}(t) / 3\right) \subset B\left(x, 2^{-1}\left(|x-A|+2^{-2} \kappa \Phi^{-1}(t) / 3\right)\right)$, by [3, Lemma 2.4],

$$
\mathbb{P}_{x_{0}}\left(Z_{\tau_{U(x)}} \in D\right) \leq \mathbb{P}_{x_{0}}\left(Z_{\tau_{U(x)}} \in B\left(x,|x-A|+2^{-2} \kappa \Phi^{-1}(t) / 3\right)^{c}\right) \leq c_{6} t^{-1} \mathbb{E}_{x_{0}}\left[\tau_{U(x)}^{Z}\right] .
$$

Since $U(x) \subset \widehat{U}\left(x_{0}\right)$, combining the above inequalities we get

$$
\mathbb{P}_{x}\left(\tau_{D}^{Z}>t\right) \leq c_{4} c_{5} c_{6} t^{-1} \mathbb{E}_{x_{0}}\left[\tau_{\widehat{U}\left(x_{0}\right)}^{Z}\right]
$$

Finally, since $D$ is $2^{-1} \kappa$-fat and $A=A_{2^{-1} \kappa \Phi^{-1}(t)}\left(x_{0}\right)$ by (5.5), using [5, Lemma 4.1], we have

$$
\mathbb{P}_{x}\left(\tau_{D}^{Z}>t\right) \leq c_{4} c_{5} c_{6} t^{-1} \mathbb{E}_{x_{0}}\left[\tau_{\widehat{U}\left(x_{0}\right)}^{Z}\right] \leq c_{7} \mathbb{P}_{x_{0}}\left(\tau_{D}^{Z}>t\right)
$$


Theorem 5.5 (Carleson estimate) Suppose that $D \subset \mathbb{R}^{d}$ is a bounded $\kappa$-fat open set with characteristics $\left(R_{1}, \kappa\right)$ satisfying the local exterior volume condition with characteristics $\left(R_{0}, C_{0}\right)$. There exists a constant $C=C\left(R_{1}, \kappa, R_{0}, C_{0}\right)>0$ such that for every $Q \in \partial D$, $r \in\left(0,\left(R_{0} \wedge R_{1}\right) / 2\right)$, and every non-negative function $f$ in $D$ that is harmonic in $D \cap B(Q, r)$ with respect to $Y^{D}$ and vanishes continuously on $\partial D \cap B(Q, r)$, we have

$$
f(x) \leq C f\left(x_{0}\right) \quad \text { for } x \in D \cap B(Q, r / 2),
$$

where $x_{0} \in D \cap B(Q, r)$ with $\delta_{D}\left(x_{0}\right) \geq \kappa r / 2$.

Proof. In this proof, the constants $\delta_{*}, \nu, \gamma, \beta_{1}, \eta$ and $c_{i}$ 's are always independent of $r$. Without loss of generality, we assume that $R_{0}=R_{1} \leq 1$ and $\operatorname{diam}(D) \leq 1$. By Theorem 4.8, it suffices to prove (5.8) for $x \in D \cap B(Q, \kappa r /(24))$.

Choose $0<\gamma<\frac{2 \gamma_{1} \delta_{1}}{d+2} \wedge \frac{1}{2}$. For any $x \in D \cap B(Q, \kappa r /(12))$, define

$$
D_{0}(x)=D \cap B\left(x, 2 \delta_{D}(x)\right), \quad B_{1}(x)=B\left(x, r^{1-\gamma} \delta_{D}(x)^{\gamma}\right)
$$

and

$$
B_{2}=B\left(x_{0}, \kappa \delta_{D}\left(x_{0}\right) / 3\right), \quad B_{3}=B\left(x_{0}, 2 \kappa \delta_{D}\left(x_{0}\right) / 3\right) .
$$

Since $x \in B(Q, \kappa r /(12))$, we have $\delta_{D}(x)<r /(12)$. By the choice of $\gamma<1 / 2$, we have that $D_{0}(x) \subset B_{1}(x)$. By Lemma 5.2, there exists $\delta_{*}=\delta_{*}\left(R_{0}, C_{0}\right)>0$ such that

$$
\mathbb{P}_{x}\left(\tau_{D_{0}(x)}=\zeta\right) \geq \mathbb{P}_{x}\left(\tau_{B\left(x, \delta_{D}(x) / 2\right)}=\zeta\right) \geq \delta_{*}, \quad x \in D \cap B(Q, \kappa r /(12)) .
$$

Further, by (2.3), (2.15) and (2.19),

$$
\begin{aligned}
\mathbb{E}_{x} \tau_{D_{0}(x)}^{Y^{D}} & \leq \int_{D_{0}(x)} G^{Y^{D}}(x, y) d y \leq c_{1} \int_{B\left(x, 2 \delta_{D}(x)\right)} g(|x-y|) d y \\
& \leq c_{2} \int_{B\left(x, 2 \delta_{D}(x)\right)} \frac{1}{|x-y|^{d} \psi\left(\Phi(|x-y|)^{-1}\right)} d y \leq \frac{c_{3}}{\psi\left(\Phi\left(\delta_{D}(x)\right)^{-1}\right)},
\end{aligned}
$$

where $g$ was defined in (5.2). By Theorem 4.8, we have

$$
f(x)<c_{4}\left(\delta_{D}(x) / r\right)^{-\beta_{1}} f\left(x_{0}\right), \quad x \in D \cap B(Q, \kappa r /(12)),
$$

where $\beta_{1}:=d+\delta_{2}>0$. Since $f$ is regular harmonic in $D_{0}(x)$ with respect to $Y^{D}$ by Lemma 5.1, for every $x \in D \cap B(Q, \kappa r /(12)))$,

$$
\begin{aligned}
f(x)= & \mathbb{E}_{x}\left[f\left(Y^{D}\left(\tau_{D_{0}(x)}\right)\right) ; Y^{D}\left(\tau_{D_{0}(x)}\right) \in B_{1}(x)\right] \\
& +\mathbb{E}_{x}\left[f\left(Y^{D}\left(\tau_{D_{0}(x)}\right)\right) ; Y^{D}\left(\tau_{D_{0}(x)}\right) \notin B_{1}(x)\right] .
\end{aligned}
$$

We first show that there exists $\eta \in\left(0,2^{-4}\right)$ such that for all $x \in D \cap B(Q, \kappa r /(12))$ with $\delta_{D}(x)<\eta r$

$$
\mathbb{E}_{x}\left[f\left(Y^{D}\left(\tau_{D_{0}(x)}\right)\right) ; Y^{D}\left(\tau_{D_{0}(x)}\right) \notin B_{1}(x)\right] \leq f\left(x_{0}\right) .
$$

Since $\gamma<\frac{1}{2}$, we have $2^{-4}<4^{-(1-\gamma)^{-1}}$. Thus for $\delta_{D}(x)<2^{-4} r$,

$$
2 \delta_{D}(x) \leq r^{1-\gamma} \delta_{D}(x)^{\gamma}-2 \delta_{D}(x)
$$


Hence, if $x \in D \cap B(Q, \kappa r /(12))$ with $\delta_{D}(x)<2^{-4} r$, then $|x-y| \leq 2|z-y|$ for $z \in D_{0}(x)$, $y \notin B_{1}(x)$.

If $z \in B_{2}$ and $y \in D \backslash B_{3}$, then it follows from Proposition 4.5 (with $\varepsilon_{0}=1$ and $\left.r=\kappa \delta_{D}\left(x_{0}\right) / 3\right)$ that $J^{Y^{D}}(z, y) \geq c_{4} J^{Y^{D}}\left(x_{0}, y\right)$. By using this estimate in the third line below, Lemma 4.6] in the fourth and $\delta_{D}\left(x_{0}\right) \geq \kappa r / 2$ in the fifth, we get that

$$
\begin{aligned}
& f\left(x_{0}\right) \geq \mathbb{E}_{x_{0}}\left[f\left(Y^{D}\left(\tau_{B_{2}}\right)\right) ; Y^{D}\left(\tau_{B_{2}}\right) \notin B_{3}\right] \\
& =\mathbb{E}_{x_{0}} \int_{0}^{\tau_{B_{2}}}\left(\int_{D \backslash B_{3}} J^{Y^{D}}\left(Y_{t}^{D}, y\right) f(y) d y\right) d t \\
& \geq c_{5} \mathbb{E}_{x_{0}}\left[\tau_{B_{2}}\right] \int_{D \backslash B_{3}} J^{Y^{D}}\left(x_{0}, y\right) f(y) d y \\
& \geq \frac{c_{6}}{\psi\left(\Phi\left(\delta_{D}\left(x_{0}\right)\right)^{-1}\right)} \int_{D \backslash B_{3}} J^{Y^{D}}\left(x_{0}, y\right) f(y) d y \\
& \geq \frac{c_{7}}{\psi\left(\Phi(r)^{-1}\right)} \int_{D \backslash B_{3}} J^{Y^{D}}\left(x_{0}, y\right) f(y) d y .
\end{aligned}
$$

Next,

$$
\begin{aligned}
\mathbb{E}_{x} & {\left[f\left(Y^{D}\left(\tau_{D_{0}(x)}\right)\right) ; Y^{D}\left(\tau_{D_{0}(x)}\right) \notin B_{1}(x)\right] } \\
= & \mathbb{E}_{x} \int_{0}^{\tau_{D_{0}(x)}} \int_{D \backslash B_{1}(x)} J^{Y^{D}}\left(Y_{t}^{D}, y\right) f(y) d y d t \\
= & \mathbb{E}_{x} \int_{0}^{\tau_{D_{0}(x)}} \int_{\left(D \backslash B_{1}(x)\right) \cap B_{3}^{c}} J^{Y^{D}}\left(Y_{t}^{D}, y\right) f(y) d y d t \\
& +\mathbb{E}_{x} \int_{0}^{\tau_{D_{0}(x)}} \int_{\left(D \backslash B_{1}(x)\right) \cap B_{3}} J^{Y^{D}}\left(Y_{t}^{D}, y\right) f(y) d y d t=: I_{1}+I_{2} .
\end{aligned}
$$

In order to estimate $I_{2}$ we first use Theorem 4.8, (2.22) and (2.23) to get

$$
I_{2} \leq c_{8} f\left(x_{0}\right) \mathbb{E}_{x} \int_{0}^{\tau_{D_{0}(x)}^{Y^{D}}} \int_{\left(D \backslash B_{1}(x)\right) \cap B_{3}} \frac{\psi\left(\Phi\left(\left|Y_{t}^{D}-y\right|\right)^{-1}\right)}{\left|Y_{t}^{D}-y\right|^{d}} d y d t .
$$

Since $|z-y| \geq \frac{1}{2}|x-y|$ for $(z, y) \in D_{0}(x) \times\left(D \backslash B_{1}(x)\right)$ and $|y-x| \geq\left|x_{0}-Q\right|-|x-Q|-\left|y-x_{0}\right|>$ $\kappa \delta_{D}\left(x_{0}\right) / 6 \geq \kappa^{2} r / 12$ for $y \in B_{3}$, we have

$$
\psi\left(\Phi(|z-y|)^{-1}\right)|z-y|^{-d} \leq c_{7} \psi\left(\Phi(r)^{-1}\right) r^{-d} \quad \text { for }(z, y) \in D_{0}(x) \times\left(B_{3} \cap\left(D \backslash B_{1}(x)\right)\right) .
$$

Therefore, by using (5.10) in the second inequality and the fact that $\delta_{D}\left(x_{0}\right)<r$ in the third, we have

$$
\begin{aligned}
& I_{2} \leq c_{9} f\left(x_{0}\right) \mathbb{E}_{x}\left[\tau_{D_{0}(x)}^{Y^{D}}\right] \int_{\left(D \backslash B_{1}(x)\right) \cap B_{3}} \frac{\psi\left(\Phi(r)^{-1}\right)}{r^{d}} d y \\
& \leq c_{10} f\left(x_{0}\right) \frac{1}{\psi\left(\Phi\left(\delta_{D}(x)\right)^{-1}\right)}\left|B_{3}\right| \frac{\psi\left(\Phi(r)^{-1}\right)}{r^{d}} \\
& \leq c_{11} f\left(x_{0}\right) \frac{\psi\left(\Phi(r)^{-1}\right)}{\psi\left(\Phi\left(\delta_{D}(x)\right)^{-1}\right)} .
\end{aligned}
$$


In order to estimate $I_{1}$ we use Propositions 5.3 and 5.4. If $w \in D_{0}(x)$, then $\delta_{D}(w) \leq r$. If we further assume $y \in D \backslash B_{1}(x)$, then $|w-y| \geq \frac{1}{2}|x-y|$, implying that $|w-y|^{d} \Phi(\mid w-$ $y \mid) \geq 2^{-d-2}|x-y|^{d} \Phi(|x-y|)$. Therefore, using Propositions 5.3 5.4 and the fact that $a \wedge(c b) \leq c(a \wedge b)$ for $a, b>0$ and $c \geq 1$,

$$
\begin{aligned}
I_{1} \leq & c_{12} \mathbb{E}_{x} \int_{0}^{\tau_{D_{0}(x)}^{Y^{D}}} \int_{\left(D \backslash B_{1}(x)\right) \cap B_{3}^{c}}\left(\int_{0}^{1} \mathbb{P}_{x_{0}}\left(\tau_{D}^{Z}>s\right) \mathbb{P}_{y}\left(\tau_{D}^{Z}>s\right)\right. \\
& \left.\times\left(\Phi^{-1}(s)^{-d} \wedge \frac{2^{d+2} s}{|x-y|^{d} \Phi(|x-y|)}\right) \nu(s) d s+\mathbb{P}_{x_{0}}\left(\tau_{D}^{Z}>1\right) \mathbb{P}_{y}\left(\tau_{D}^{Z}>1\right)\right) f(y) d y d t \\
\leq & 2^{d+2} c_{12} \mathbb{E}_{x}\left[\tau_{D_{0}(x)}^{Y^{D}}\right] \int_{\left(D \backslash B_{1}(x)\right) \cap B_{3}^{c}}\left(\int_{0}^{1} \mathbb{P}_{x_{0}}\left(\tau_{D}^{Z}>s\right) \mathbb{P}_{y}\left(\tau_{D}^{Z}>s\right)\right. \\
& \left.\times\left(\Phi^{-1}(s)^{-d} \wedge \frac{s}{|x-y|^{d} \Phi(|x-y|)}\right) \nu(s) d s+\mathbb{P}_{x_{0}}\left(\tau_{D}^{Z}>1\right) \mathbb{P}_{y}\left(\tau_{D}^{Z}>1\right)\right) f(y) d y .
\end{aligned}
$$

Recall that $x \in B(Q, \kappa r /(24))$. For $y \in D \backslash B_{1}(x)$ we have $|y-x| \geq r^{1-\gamma} \delta_{D}(x)^{\gamma}$, and therefore

$$
\left|y-x_{0}\right| \leq|y-x|+r \leq|y-x|+r^{\gamma} \delta_{D}(x)^{-\gamma}|y-x| \leq 2 r^{\gamma} \delta_{D}(x)^{-\gamma}|y-x| .
$$

Thus by (2.9), we have $\Phi\left(\left|y-x_{0}\right|\right) \leq\left(2 r^{\gamma} \delta_{D}(x)^{-\gamma}\right)^{2} \Phi(|x-y|)$. Hence, by using (5.10) in the first inequality below, the fact that $a \wedge(c b) \leq c(a \wedge b)$ for $a, b>0$ and $c \geq 1$ in the second, Proposition 5.3 in the third, and (5.14) in the last inequality, we have

$$
\begin{aligned}
I_{1} \leq & \frac{c_{13}}{\psi\left(\Phi\left(\delta_{D}(x)\right)^{-1}\right)} \int_{\left(D \backslash B_{1}(x)\right) \cap B_{3}^{c}}\left(\int_{0}^{1} \mathbb{P}_{x_{0}}\left(\tau_{D}^{Z}>s\right) \mathbb{P}_{y}\left(\tau_{D}^{Z}>s\right)\right. \\
& \left.\times\left(\Phi^{-1}(s)^{-d} \wedge \frac{\left(2 r / \delta_{D}(x)\right)^{\gamma(d+2)} s}{\left|x_{0}-y\right|^{d} \Phi\left(\left|x_{0}-y\right|\right)}\right) \nu(s) d s+\mathbb{P}_{x_{0}}\left(\tau_{D}^{Z}>1\right) \mathbb{P}_{y}\left(\tau_{D}^{Z}>1\right)\right) f(y) d y \\
\leq & \frac{c_{13} 2^{\gamma(d+2)}}{\psi\left(\Phi\left(\delta_{D}(x)\right)^{-1}\right)}\left(\frac{\delta_{D}(x)}{r}\right)^{-\gamma(d+2)} \int_{D \backslash B_{3}}\left(\int_{0}^{1} \mathbb{P}_{x_{0}}\left(\tau_{D}^{Z}>s\right) \mathbb{P}_{y}\left(\tau_{D}^{Z}>s\right)\right. \\
& \left.\times\left(\Phi^{-1}(s)^{-d} \wedge \frac{s}{\left|x_{0}-y\right|^{d} \Phi\left(\left|x_{0}-y\right|\right)}\right) \nu(s) d s+\mathbb{P}_{x_{0}}\left(\tau_{D}^{Z}>1\right) \mathbb{P}_{y}\left(\tau_{D}^{Z}>1\right)\right) f(y) d y \\
\leq & \frac{c_{14}}{\psi\left(\Phi\left(\delta_{D}(x)\right)^{-1}\right)}\left(\frac{\delta_{D}(x)}{r}\right)^{-\gamma(d+2)} \int_{D \backslash B_{3}} J^{Y^{D}}\left(x_{0}, y\right) f(y) d y \\
\leq & c_{15} \frac{\psi\left(\Phi(r)^{-1}\right)}{\psi\left(\Phi\left(\delta_{D}(x)\right)^{-1}\right)}\left(\frac{\delta_{D}(x)}{r}\right)^{-\gamma(d+2)} f\left(x_{0}\right) .
\end{aligned}
$$

Combining (5.15), (5.17) and (5.19), we obtain

$$
\begin{aligned}
& \mathbb{E}_{x}\left[f\left(Y^{D}\left(\tau_{D_{0}(x)}\right)\right) ; Y^{D}\left(\tau_{D_{0}(x)}\right) \notin B_{1}(x)\right] \\
& \leq c_{16} f\left(x_{0}\right) \frac{\psi\left(\Phi(r)^{-1}\right)}{\psi\left(\Phi\left(\delta_{D}(x)\right)^{-1}\right)}\left(\left(\frac{\delta_{D}(x)}{r}\right)^{-\gamma(d+2)}+1\right) \\
& \leq c_{17} f\left(x_{0}\right)\left(\frac{\delta_{D}(x)}{r}\right)^{2 \delta_{1} \gamma_{1}}\left(\left(\frac{\delta_{D}(x)}{r}\right)^{-\gamma(d+2)}+1\right) .
\end{aligned}
$$


Since $2 \gamma_{1} \delta_{1}-(d+2) \gamma>0$, we can choose $\eta \in\left(0,2^{-4}\right)$ so that

$$
c_{17}\left(\eta^{2 \gamma_{1} \delta_{1}-(d+2) \gamma}+\eta^{2 \gamma_{1} \delta_{1}}\right) \leq 1 .
$$

Then for $x \in D \cap B(Q, \kappa r /(12))$ with $\delta_{D}(x)<\eta r$, we have by (5.20),

$$
\mathbb{E}_{x}\left[f\left(Y^{D}\left(\tau_{D_{0}(x)}\right)\right) ; Y^{D}\left(\tau_{D_{0}(x)}\right) \notin B_{1}(x)\right] \leq c_{17} f\left(x_{0}\right)\left(\eta^{2 \gamma_{1} \delta_{1}-(d+2) \gamma}+\eta^{2 \gamma_{1} \delta_{1}}\right) \leq f\left(x_{0}\right) .
$$

This completes the proof of (5.13).

With (5.13), one can prove the Carleson estimate (5.8) for $x \in D \cap B(Q, \kappa r /(24))$ by a method of contradiction. Since this part of the proof is the same as the corresponding part in the proof of [18, Theorem 5.4], we omit the details.

\section{Green function and exit time estimates}

In this section, we assume that $D$ is a bounded $C^{1,1}$ open set in $\mathbb{R}^{d}, d \geq 2$, with $C^{1,1}$ characteristics $(R, \Lambda)$. The first goal of this section is to derive sharp two-sided estimates for $G^{Y^{D}}$. See [15, Theorem 3.1] for the corresponding result for killed subordinate Brownian motion.

Recall that $\Phi(r)=\frac{1}{\phi\left(r^{-2}\right)}$. For $t>0$ and $x, y \in D$, let

$$
r(t, x, y):=\left(\frac{\Phi\left(\delta_{D}(x)\right)^{1 / 2}}{t^{1 / 2}} \wedge 1\right)\left(\frac{\Phi\left(\delta_{D}(y)\right)^{1 / 2}}{t^{1 / 2}} \wedge 1\right)\left(\Phi^{-1}(t)^{-d} \wedge \frac{t}{|x-y|^{d} \Phi(|x-y|)}\right)
$$

Combining [5, Corollary 1.6] with (4.2) we get that for every $T>0$ there exist $C_{1}=$ $C_{1}\left(T, R, \Lambda, a_{2}, a_{2}, \delta_{1}, \delta_{2}\right) \geq 1$ and $C_{2}=C_{2}\left(T, R, \Lambda, a_{2}, a_{2}, \delta_{1}, \delta_{2}, \operatorname{diam}(D)\right) \geq 1$ such that

$$
C_{1}^{-1} r(t, x, y) \leq p^{D}(t, x, y) \leq C_{1} r(t, x, y)
$$

for all $(t, x, y) \in(0, T] \times D \times D$, and

$$
C_{2}^{-1} e^{-\lambda_{1} t} \Phi\left(\delta_{D}(x)\right)^{1 / 2} \Phi\left(\delta_{D}(y)\right)^{1 / 2} \leq p^{D}(t, x, y) \leq C_{2} e^{-\lambda_{1} t} \Phi\left(\delta_{D}(x)\right)^{1 / 2} \Phi\left(\delta_{D}(y)\right)^{1 / 2}
$$

for all $(t, x, y) \in(T, \infty) \times D \times D$. Here $-\lambda_{1}<0$ is the largest eigenvalue of the infinitesimal generator of $Z^{D}$.

It follows easily from (2.2) and (2.5) that

$$
b_{2}^{-1}\left(\frac{s}{t}\right)^{1-\gamma_{2}} \leq \frac{v(t)}{v(s)} \leq b_{1}^{-1}\left(\frac{s}{t}\right)^{1-\gamma_{1}} \quad, \quad 0<t \leq s \leq 1 .
$$

It is shown in [5, Lemma 7.1] that

$$
\left(\frac{\Phi\left(\delta_{D}(x)\right)^{1 / 2} \Phi\left(\delta_{D}(y)\right)^{1 / 2}}{\Phi(|x-y|)} \wedge 1\right) \asymp^{c}\left(\frac{\Phi\left(\delta_{D}(x)\right)^{1 / 2}}{\Phi(|x-y|)^{1 / 2}} \wedge 1\right)\left(\frac{\Phi\left(\delta_{D}(y)\right)^{1 / 2}}{\Phi(|x-y|)^{1 / 2}} \wedge 1\right) .
$$


Lemma 6.1 Let $f:[0, \infty) \rightarrow[0, \infty)$ be a decreasing function. For any $T>0$, there exists $C=C(f, \phi, \operatorname{diam}(D), T)>0$ such that for all $x, y \in D$,

$$
\int_{T}^{\infty} p^{D}(t, x, y) f(t) d t \leq C\left(\frac{\Phi\left(\delta_{D}(x)\right)^{1 / 2}}{\Phi(|x-y|)^{1 / 2}} \wedge 1\right)\left(\frac{\Phi\left(\delta_{D}(y)\right)^{1 / 2}}{\Phi(|x-y|)^{1 / 2}} \wedge 1\right) \frac{\Phi(|x-y|) f(\Phi(|x-y|))}{|x-y|^{d}} .
$$

Proof. For $x, y \in D$, let $a(x, y):=\Phi\left(\delta_{D}(x)\right)^{1 / 2} \Phi\left(\delta_{D}(y)\right)^{1 / 2}$ and

$$
b(x, y):=\left(\frac{a(x, y)}{\Phi(|x-y|)} \wedge 1\right) \frac{\Phi(|x-y|) f(\Phi(|x-y|))}{|x-y|^{d}} .
$$

If $a(x, y) \leq \Phi(|x-y|)$, then

$$
b(x, y)=a(x, y) \frac{f(\Phi(|x-y|))}{|x-y|^{d}} \geq \frac{f(\Phi(\operatorname{diam}(D)))}{\operatorname{diam}(D)^{d}} a(x, y) .
$$

If $a(x, y)>\Phi(|x-y|)$, then by (2.12),

$$
\begin{aligned}
& b(x, y)=f(\Phi(|x-y|)) \frac{\Phi(|x-y|)}{|x-y|^{d}} \\
& \geq f(\Phi(\operatorname{diam}(D))) \frac{\Phi(\operatorname{diam}(D))}{\operatorname{diam}(D)^{d}} \geq \frac{f(\Phi(\operatorname{diam}(D)))}{\operatorname{diam}(D)^{d}} a(x, y) .
\end{aligned}
$$

Since by (6.3),

$$
\int_{T}^{\infty} p^{D}(t, x, y) f(t) d t \leq c_{3} a(x, y) f(T) \int_{T}^{\infty} e^{-\lambda_{1} t} d t \leq c_{4} a(x, y)
$$

the claim now follows from (6.6), (6.7) and (6.5).

Lemma 6.2 Let $T>\Phi(\operatorname{diam}(D))$. There exists a constant $C=C\left(T, a_{2}, \delta_{2}\right) \geq 1$ such that for all $x, y \in D$,

$$
\int_{\Phi(|x-y|)}^{T} r(t, x, y) d t \leq C\left(\frac{\Phi\left(\delta_{D}(x)\right)^{1 / 2}}{\Phi(|x-y|)^{1 / 2}} \wedge 1\right)\left(\frac{\Phi\left(\delta_{D}(y)\right)^{1 / 2}}{\Phi(|x-y|)^{1 / 2}} \wedge 1\right) \frac{\Phi(|x-y|)}{|x-y|^{d}}
$$

Proof. First note that by (2.10), for $\Phi(|x-y|) \leq t \leq T$,

$$
\Phi^{-1}(t)^{-d} \leq C_{T}^{d}|x-y|^{-d} \Phi(|x-y|)^{d / 2 \delta_{2}} t^{-d / 2 \delta_{2}} .
$$

Therefore, using (4.4) we have

$$
\begin{aligned}
& \int_{\Phi(|x-y|)}^{T} r(t, x, y) d t \\
& \quad \leq \int_{\Phi(|x-y|)}^{T}\left(\frac{\Phi\left(\delta_{D}(x)\right)^{1 / 2}}{\Phi(|x-y|)^{1 / 2}} \wedge 1\right)\left(\frac{\Phi\left(\delta_{D}(y)\right)^{1 / 2}}{\Phi(|x-y|)^{1 / 2}} \wedge 1\right) \Phi^{-1}(t)^{-d} d t \\
& \quad \leq \quad c_{1}\left(\frac{\Phi\left(\delta_{D}(x)\right)^{1 / 2}}{\Phi(|x-y|)^{1 / 2}} \wedge 1\right)\left(\frac{\Phi\left(\delta_{D}(y)\right)^{1 / 2}}{\Phi(|x-y|)^{1 / 2}} \wedge 1\right) \frac{\Phi(|x-y|)^{d / 2 \delta_{2}}}{|x-y|^{d}} \int_{\Phi(|x-y|)}^{\infty} t^{-d / 2 \delta_{2}} d t
\end{aligned}
$$




$$
=c_{2}\left(\frac{\Phi\left(\delta_{D}(x)\right)^{1 / 2}}{\Phi(|x-y|)^{1 / 2}} \wedge 1\right)\left(\frac{\Phi\left(\delta_{D}(y)\right)^{1 / 2}}{\Phi(|x-y|)^{1 / 2}} \wedge 1\right) \frac{\Phi(|x-y|)}{|x-y|^{d}}
$$

Lemmas 6.1 and 6.2 will also be used in Section 8 .

Lemma 6.3 There exists a constant $C \geq 1$ such that for all $x, y \in D$,

$$
\begin{aligned}
& \int_{0}^{\Phi(|x-y|)} r(t, x, y) v(t) d t \\
\asymp^{C} & \left(\frac{\Phi\left(\delta_{D}(x)\right)^{1 / 2}}{\Phi(|x-y|)^{1 / 2}} \wedge 1\right)\left(\frac{\Phi\left(\delta_{D}(y)\right)^{1 / 2}}{\Phi(|x-y|)^{1 / 2}} \wedge 1\right) \frac{\Phi(|x-y|) v(\Phi(|x-y|))}{|x-y|^{d}} .
\end{aligned}
$$

Proof. By using (6.1), (4.4) and (6.4) in the second line, the change of variable $s=$ $\Phi(|x-y|) t^{-1}$ in the third line, the fact that $(s a) \wedge 1 \leq s(a \wedge 1)$ for every $a>0$ and $s \geq 1$ in the fourth line, we get

$$
\begin{aligned}
& \int_{0}^{\Phi(|x-y|)} r(t, x, y) v(t) d t \\
& \leq c_{1} \frac{v(\Phi(|x-y|)) \Phi(|x-y|)^{-\gamma_{1}+1}}{|x-y|^{d} \Phi(|x-y|)} \int_{0}^{\Phi(|x-y|)}\left(\frac{\Phi\left(\delta_{D}(x)\right)^{1 / 2}}{t^{1 / 2}} \wedge 1\right)\left(\frac{\Phi\left(\delta_{D}(y)\right)^{1 / 2}}{t^{1 / 2}} \wedge 1\right) t^{\gamma_{1}} d t \\
& =c_{1} \frac{v(\Phi(|x-y|)) \Phi(|x-y|)}{|x-y|^{d}} \int_{1}^{\infty}\left(\frac{s^{1 / 2} \Phi\left(\delta_{D}(x)\right)^{1 / 2}}{\Phi(|x-y|)^{1 / 2}} \wedge 1\right)\left(\frac{s^{1 / 2} \Phi\left(\delta_{D}(y)\right)^{1 / 2}}{\Phi(|x-y|)^{1 / 2}} \wedge 1\right) s^{-\gamma_{1}-2} d s \\
& \leq c_{1}\left(\frac{\Phi\left(\delta_{D}(x)\right)^{1 / 2}}{\Phi(|x-y|)^{1 / 2}} \wedge 1\right)\left(\frac{\Phi\left(\delta_{D}(y)\right)^{1 / 2}}{\Phi(|x-y|)^{1 / 2}} \wedge 1\right) \frac{v(\Phi(|x-y|)) \Phi(|x-y|)}{|x-y|^{d}} \int_{1}^{\infty} s^{-\gamma_{1}-1} d s .
\end{aligned}
$$

Since $\int_{1}^{\infty} s^{-\gamma_{1}-1} d s<\infty$, this proves the upper bound.

On the other hand, by using (6.1), (4.4) and the fact that $v$ is decreasing in the second line, we get

$$
\begin{aligned}
& \int_{0}^{\Phi(|x-y|)} r(t, x, y) v(t) d t \\
& \quad \geq \frac{v(\Phi(|x-y|))}{|x-y|^{d} \Phi(|x-y|)} \int_{0}^{\Phi(|x-y|)}\left(\frac{\Phi\left(\delta_{D}(x)\right)^{1 / 2}}{t^{1 / 2}} \wedge 1\right)\left(\frac{\Phi\left(\delta_{D}(y)\right)^{1 / 2}}{t^{1 / 2}} \wedge 1\right) t d t \\
& \quad \geq\left(\frac{\Phi\left(\delta_{D}(x)\right)^{1 / 2}}{\Phi(|x-y|)^{1 / 2}} \wedge 1\right)\left(\frac{\Phi\left(\delta_{D}(y)\right)^{1 / 2}}{\Phi(|x-y|)^{1 / 2}} \wedge 1\right) \frac{v(\Phi(|x-y|))}{|x-y|^{d} \Phi(|x-y|)} \int_{0}^{\Phi(|x-y|)} t d t \\
& \quad=\frac{1}{2}\left(\frac{\Phi\left(\delta_{D}(x)\right)^{1 / 2}}{\Phi(|x-y|)^{1 / 2}} \wedge 1\right)\left(\frac{\Phi\left(\delta_{D}(y)\right)^{1 / 2}}{\Phi(|x-y|)^{1 / 2}} \wedge 1\right) \frac{v(\Phi(|x-y|)) \Phi(|x-y|)}{|x-y|^{d}} .
\end{aligned}
$$

Recall the function $g$ defined in (5.2). Note that $g$ satisfies the doubling property near 0 by (2.3) and, by (2.13), $g$ is a decreasing function. 
Theorem 6.4 There exists a constant $C \geq 1$ such that for all $x, y \in D$,

$$
G^{Y^{D}}(x, y) \asymp^{C}\left(\frac{\Phi\left(\delta_{D}(x)\right)^{1 / 2}}{\Phi(|x-y|)^{1 / 2}} \wedge 1\right)\left(\frac{\Phi\left(\delta_{D}(y)\right)^{1 / 2}}{\Phi(|x-y|)^{1 / 2}} \wedge 1\right) g(|x-y|) .
$$

Proof. Let $T=2 \Phi(\operatorname{diam}(D))$. By (2.18), (6.1), (6.2) and (6.3),

$$
\begin{aligned}
& G^{Y^{D}}(x, y)=\int_{0}^{\infty} p^{D}(t, x, y) v(t) d t \\
& \leq c_{1}\left(\int_{0}^{\Phi(|x-y|)} r(t, x, y) v(t) d t+\int_{\Phi(|x-y|)}^{T} r(t, x, y) v(t) d t+\int_{T}^{\infty} p^{D}(t, x, y) v(t) d t\right) \\
& =: c_{1}\left(I_{1}+I_{2}+I_{3}\right) .
\end{aligned}
$$

By Lemmas 6.16.3 and the fact that $v$ is decreasing, for each $j=1,2,3$,

$$
\begin{aligned}
I_{j} & \leq c_{2}\left(\frac{\Phi\left(\delta_{D}(x)\right)^{1 / 2}}{\Phi(|x-y|)^{1 / 2}} \wedge 1\right)\left(\frac{\Phi\left(\delta_{D}(y)\right)^{1 / 2}}{\Phi(|x-y|)^{1 / 2}} \wedge 1\right) \frac{\Phi(|x-y|) v(\Phi(|x-y|))}{|x-y|^{d}} \\
& \leq c_{3}\left(\frac{\Phi\left(\delta_{D}(x)\right)^{1 / 2}}{\Phi(|x-y|)^{1 / 2}} \wedge 1\right)\left(\frac{\Phi\left(\delta_{D}(y)\right)^{1 / 2}}{\Phi(|x-y|)^{1 / 2}} \wedge 1\right) g(|x-y|) .
\end{aligned}
$$

Here the last line follows from (2.5) and the definition of $g$. For the lower bound we use $G^{Y^{D}}(x, y) \geq c_{4} I_{1}$ and Lemma 6.3.

Let $Q \in \partial D$ and choose $\varphi_{Q}$ as in Definition 3.1. Define $\rho_{Q}(x):=x_{d}-\varphi_{Q}(\widetilde{x})$, where $\left(\widetilde{x}, x_{d}\right)$ are the coordinates of $x$ in $C S_{Q}$. Note that for every $Q \in \partial D$ and $x \in B(Q, R) \cap D$, we have

$$
\left(1+\Lambda^{2}\right)^{-1 / 2} \rho_{Q}(x) \leq \delta_{D}(x) \leq \rho_{Q}(x)
$$

We define for $r_{1}, r_{2}>0$,

$$
D_{Q}\left(r_{1}, r_{2}\right):=\left\{y \in D: r_{1}>\rho_{Q}(y)>0,|\widetilde{y}|<r_{2}\right\} .
$$

Let $\kappa_{0}=\left(1+(1+\Lambda)^{2}\right)^{-1 / 2}$. It is well known (see, for instance, [23, Lemma 2.2]) that there exists $L=L(R, \Lambda, d)>0$ such that for every $z \in \partial D$ and $r \leq \kappa_{0} R$, one can find a $C^{1,1}$ domain $V_{Q}(r)$ with characteristics $(r R / L, \Lambda L / r)$ such that $D_{Q}(r / 2, r / 2) \subset V_{Q}(r) \subset D_{Q}(r, r)$. In this and the following two sections, given a $C^{1,1}$ open set $D, V_{Q}(r)$ always refers to the $C^{1,1}$ domain above.

It is easy to see that for every $Q \in \partial D$ and $r \leq \kappa_{0} R$,

$$
V_{Q}(r) \subset D_{Q}(r, r) \subset D \cap B\left(Q, r / \kappa_{0}\right) .
$$

In fact, for all $y \in D_{Q}(r, r)$,

$$
|y|^{2}=|\widetilde{y}|^{2}+\left|y_{d}\right|^{2}<r^{2}+\left(\left|y_{d}-\varphi_{Q}(\widetilde{y})\right|+\left|\varphi_{Q}(\widetilde{y})\right|\right)^{2}<\left(1+(1+\Lambda)^{2}\right) r^{2} .
$$

For any $r \leq 1$, let $\phi^{r}$ be defined by $\phi^{r}(\lambda):=\phi\left(\lambda r^{-2}\right) / \phi\left(r^{-2}\right)$. Then $\phi^{r}$ is also a complete Bernstein function. Let $S^{r}$ be a subordinator independent of the Brownian motion $W$ and let $Z^{r}$ be defined by $Z_{t}^{r}:=W_{S_{t}^{r}}$. Then, cf. [14, p. 247], $Z^{r}$ is identical in law to the process 
$\left\{r^{-1} Z_{t / \phi\left(r^{-2}\right)}\right\}_{t \geq 0}$. Let $p^{r}(t, x, y)$ be the transition density of $Z^{r}$. For any open set $U$, let $p^{r, U}(t, x, y)$ be the transition density of $Z^{r, U}$, the subprocess of $Z^{r}$ killed upon exiting $U$.

By the fact that $r^{-1} V_{Q}(r)$ is a $C^{1,1}$ open set with $C^{1,1}$ characteristics $(R / L, \Lambda L)$, we get from (6.2)-(6.3) that there exists $C_{3} \geq 1$ such that

$$
C_{3}^{-1} \widetilde{r}(t, x, y) \leq p^{r, r^{-1} V_{Q}(r)}(t, x, y) \leq C_{3} \widetilde{r}(t, x, y)
$$

for all $(t, x, y) \in\left(0, a_{2}\left(2 / \kappa_{0}\right)^{2 \delta_{2}}\right] \times r^{-1} V_{Q}(r) \times r^{-1} V_{Q}(r)$, where

$$
\begin{aligned}
& \widetilde{r}(t, x, y) \\
& :=\left(\frac{\Phi\left(\delta_{r^{-1} V_{Q}(r)}(x)\right)^{1 / 2}}{t^{1 / 2}} \wedge 1\right)\left(\frac{\Phi\left(\delta_{r^{-1} V_{Q}(r)}(y)\right)^{1 / 2}}{t^{1 / 2}} \wedge 1\right)\left(\Phi^{-1}(t)^{-d} \wedge \frac{t}{|x-y|^{d} \Phi(|x-y|)}\right) .
\end{aligned}
$$

By the scaling property mentioned in the paragraph above we get that

$$
p^{V_{Q}(r)}(t, x, y)=r^{-d} p^{r, r^{-1} V_{Q}(r)}\left(\phi\left(r^{-2}\right) t, r^{-1} x, r^{-1} y\right)=r^{-d} p^{r, r^{-1} V_{Q}(r)}\left(\Phi(r)^{-1} t, r^{-1} x, r^{-1} y\right) .
$$

Thus, since $\Phi\left(2 r / \kappa_{0}\right) \leq a_{2}\left(2 / \kappa_{0}\right)^{2 \delta_{2}} \Phi(r)$ by (2.1), we have

$$
C_{3}^{-1} \widehat{r}(t, x, y) \leq p^{V_{Q}(r)}(t, x, y) \leq C_{3} \widehat{r}(t, x, y)
$$

for all $(t, x, y) \in\left(0, \Phi\left(2 r / \kappa_{0}\right)\right] \times V_{Q}(r) \times V_{Q}(r)$, where

$$
\begin{aligned}
& \widehat{r}(t, x, y) \\
& :=\left(\frac{\Phi\left(\delta_{V_{Q}(r)}(x)\right)^{1 / 2}}{t^{1 / 2}} \wedge 1\right)\left(\frac{\Phi\left(\delta_{V_{Q}(r)}(y)\right)^{1 / 2}}{t^{1 / 2}} \wedge 1\right)\left(\Phi^{-1}(t)^{-d} \wedge \frac{t}{|x-y|^{d} \Phi(|x-y|)}\right) .
\end{aligned}
$$

Since $\operatorname{diam}\left(V_{Q}(r)\right) \leq 2 r / \kappa_{0}$, using the lower bound in (6.14) and following the argument in (6.9), one can easily prove the following

Proposition 6.5 There exists $C=C(R, \Lambda) \geq 1$ such that for all $Q \in \partial D, r \leq \kappa_{0} R$ and $x, y \in V_{Q}(r)$,

$$
G^{Y^{V_{Q}(r)}}(x, y) \geq C^{-1}\left(\frac{\Phi\left(\delta_{V_{Q}(r)}(x)\right)^{1 / 2}}{\Phi(|x-y|)^{1 / 2}} \wedge 1\right)\left(\frac{\Phi\left(\delta_{V_{Q}(r)}(y)\right)^{1 / 2}}{\Phi(|x-y|)^{1 / 2}} \wedge 1\right) g(|x-y|) .
$$

For simplicity, let $\tau_{D}:=\zeta$. Then $\mathbb{E}_{x} \tau_{D}=\int_{D} G^{Y^{D}}(x, y) d y$. The final goal of the section is to give sharp two-sided estimates on $\mathbb{E}_{x} \tau_{D}$. Lemmas 6.7 and 6.8 below will be used in Section 9 ,

Lemma 6.6 If $\gamma_{1}>1 / 2$, then there exists $C=C(D, \phi, \psi)>0$ such that

$$
\mathbb{E}_{x} \tau_{D} \asymp^{C} \Phi\left(\delta_{D}(x)\right)^{1 / 2}, \quad x \in D .
$$

Proof. Let $T=\operatorname{diam}(D)$. By using Theorem 6.4 in the first inequality, (2.2) in the penultimate inequality, and (2.1) and $\gamma_{1}>1 / 2$ in the last inequality, we get that

$$
\mathbb{E}_{x} \tau_{D}=\int_{D} G^{Y^{D}}(x, y) d y
$$




$$
\begin{aligned}
& \leq c_{1} \Phi\left(\delta_{D}(x)\right)^{1 / 2} \int_{D} \frac{1}{\Phi(|x-y|)^{1 / 2}|x-y|^{d} \psi\left(\Phi(|x-y|)^{-1}\right)} d y \\
& \leq c_{2} \Phi\left(\delta_{D}(x)\right)^{1 / 2} \int_{B(x, T)} \frac{1}{\Phi(|x-y|)^{1 / 2}|x-y|^{d} \psi\left(\Phi(|x-y|)^{-1}\right)} d y \\
& \leq c_{3} \Phi\left(\delta_{D}(x)\right)^{1 / 2} \int_{0}^{T} \frac{1}{\Phi(s)^{1 / 2} s \psi\left(\Phi(s)^{-1}\right)} d s \\
& \leq c_{4} \frac{\Phi\left(\delta_{D}(x)\right)^{1 / 2}}{\Phi(T)^{1 / 2} \psi\left(\Phi(T)^{-1}\right)} \int_{0}^{T} \frac{d s}{s}\left(\frac{\Phi(T)}{\Phi(s)}\right)^{\frac{1}{2}-\gamma_{1}} \\
& \leq c_{5} \Phi\left(\delta_{D}(x)\right)^{1 / 2} .
\end{aligned}
$$

For the lower bound, recall that any $C^{1,1}$, open set satisfies the interior ball condition with some radius $\widehat{r}$. Let $a:=(\operatorname{diam}(D) / 10) \wedge \widehat{r}$ and $D_{a}:=\left\{y \in D: \delta_{D}(y)>a\right\}$.

Case (i): $\delta_{D}(x)<a$. Then $B\left(x, \delta_{D}(x) / 2\right) \cap D_{2 a}=\emptyset$. For $y \in D \backslash B\left(x, \delta_{D}(x) / 2\right)$, we have

$$
2|x-y| \geq \delta_{D}(x), \quad 3|x-y| \geq \delta_{D}(x)+|x-y| \geq \delta_{D}(y),
$$

hence by Theorem 6.4,

$$
G^{Y^{D}}(x, y) \geq c_{6} \frac{\Phi\left(\delta_{D}(x)\right)^{1 / 2} \Phi\left(\delta_{D}(y)\right)^{1 / 2}}{\Phi(|x-y|)} g(|x-y|) .
$$

Recall from (2.11) that $\theta(t):=\Phi(t) \psi\left(\Phi(t)^{-1}\right)$ is increasing, hence $t \mapsto t^{d} \theta(t)$ is also increasing. Thus we have

$$
\begin{aligned}
& \mathbb{E}_{x} \tau_{D} \geq c_{7} \int_{D_{2 a}} \Phi\left(\delta_{D}(x)\right)^{1 / 2} \Phi\left(\delta_{D}(y)\right)^{1 / 2} \frac{1}{\Phi(|x-y|)|x-y|^{d} \psi\left(\Phi(|x-y|)^{-1}\right)} \\
& \geq c_{7} \Phi\left(\delta_{D}(x)\right)^{1 / 2} \Phi(2 a)^{1 / 2} \int_{D_{2 a}} \frac{d y}{|x-y|^{d} \theta(|x-y|)} \\
& \geq c_{8} \Phi\left(\delta_{D}(x)\right)^{1 / 2} \frac{\left|D_{2 a}\right|}{(\operatorname{diam}(D))^{d} \theta(\operatorname{diam}(D))} \\
& =c_{9} \Phi\left(\delta_{D}(x)\right)^{1 / 2}
\end{aligned}
$$

Case (ii): $\delta_{D}(x) \geq a$. Since $T \geq \delta_{D}(x) \geq a$, we have

$$
\mathbb{E}_{x} \tau_{D} \geq c_{10} \int_{B\left(x, \delta_{D}(x) / 2\right)} g(|x-y|) \geq c_{11} \int_{0}^{a / 2} \frac{d s}{s \psi\left(\Phi(s)^{-1}\right)} \geq c_{12} \Phi\left(\delta_{D}(x)\right)^{1 / 2} .
$$

Lemma 6.7 If $\gamma_{2}<1 / 2$, then there exists $C=C(D, \phi, \psi)>0$ such that

$$
\mathbb{E}_{x} \tau_{D} \asymp^{C} \frac{1}{\psi\left(\Phi\left(\delta_{D}(x)\right)^{-1}\right)}, \quad x \in D
$$


Proof. Let $T=\operatorname{diam}(D)$. By using (2.2) and (2.1), we have

$$
\begin{aligned}
& \mathbb{E}_{x} \tau_{D} \leq c_{1} \int_{D}\left(\frac{\Phi\left(\delta_{D}(x)\right)^{1 / 2}}{\Phi(|x-y|)^{1 / 2}} \wedge 1\right) g(|x-y|) d y \\
& =c_{1} \int_{D \cap\left\{|x-y|<\delta_{D}(x)\right\}} g(|x-y|) d y \\
& \quad+c_{1} \int_{D \cap\left\{|x-y| \geq \delta_{D}(x)\right\}} \frac{\Phi\left(\delta_{D}(x)\right)^{1 / 2}}{\Phi(|x-y|)^{1 / 2}|x-y|^{d} \psi\left(\Phi(|x-y|)^{-1}\right)} d y \\
& \leq c_{2} \frac{1}{\psi\left(\Phi\left(\delta_{D}(x)\right)^{-1}\right)}+\frac{c_{3}}{\psi\left(\Phi\left(\delta_{D}(x)\right)^{-1}\right)} \int_{\delta_{D}(x)}^{T} \frac{\Phi\left(\delta_{D}(x)\right)^{1 / 2} \psi\left(\Phi\left(\delta_{D}(x)\right)^{-1}\right)}{\Phi(s)^{1 / 2} s \psi\left(\Phi(s)^{-1}\right)} d s \\
& \leq c_{2} \frac{1}{\psi\left(\Phi\left(\delta_{D}(x)\right)^{-1}\right)}+\frac{c_{4}}{\psi\left(\Phi\left(\delta_{D}(x)\right)^{-1}\right)} \int_{\delta_{D}(x)}^{T} \frac{1}{s}\left(\frac{\Phi\left(\delta_{D}(x)\right)}{\Phi(s)}\right)^{\frac{1}{2}-\gamma_{2}} d s \\
& \leq c_{5} \frac{1}{\psi\left(\Phi\left(\delta_{D}(x)\right)^{-1}\right)} .
\end{aligned}
$$

The lower bound is an immediate consequence of Lemma 4.6:

$$
\mathbb{E}_{x} \tau_{D} \geq \mathbb{E}_{x} \tau_{B\left(x, \delta_{D}(x)\right)} \geq c_{6} \frac{1}{\psi\left(\Phi\left(\delta_{D}(x)\right)^{-1}\right)}
$$

Lemma 6.8 If $\psi(\lambda)=\lambda^{1 / 2}$, then there exists $C=C(D, \phi)>0$ such that

$$
\mathbb{E}_{x} \tau_{D} \asymp^{C} \Phi\left(\delta_{D}(x)\right)^{1 / 2} \log \left(1 / \delta_{D}(x)\right), \quad x \in D .
$$

Proof. By following the proof of the upper bound in Lemma 6.7, we obtain

$$
\begin{aligned}
& \mathbb{E}_{x} \tau_{D} \leq c_{1} \frac{1}{\psi\left(\Phi\left(\delta_{D}(x)\right)^{-1}\right)}+c_{2} \Phi\left(\delta_{D}(x)\right)^{1 / 2} \int_{\delta_{D}(x)}^{\operatorname{diam}(D)} \frac{1}{\Phi(s)^{1 / 2} s\left(\Phi(s)^{-1}\right)^{1 / 2}} d s \\
& =c_{1} \frac{1}{\psi\left(\Phi\left(\delta_{D}(x)\right)^{-1}\right)}+c_{2} \Phi\left(\delta_{D}(x)\right)^{1 / 2} \log \left(\operatorname{diam}(D) / \delta_{D}(x)\right) \\
& \leq c_{3} \Phi\left(\delta_{D}(x)\right)^{1 / 2} \log \left(1 / \delta_{D}(x)\right) .
\end{aligned}
$$

For the lower bound, as one can see from the end of the proof of Lemma 6.7, we only need to consider $x$ close to the boundary. Since $D$ is $C^{1,1}$, there exists a constant $L>0$ such that for every $x \in D$ with $\delta_{D}(x) \leq L / 2$, one can find a cone $\mathcal{C}$ with vertex in $x$, pointing inward, with height $L$, and aperture not depending on $x$. Moreover, such a cone $\mathcal{C}$ can be chosen so that $\mathcal{C} \subset\left\{y \in D:|x-y| \leq \delta_{D}(y)\right\}$. Then for $y \in \mathcal{C} \cap\left\{y \in D: \delta_{D}(x) \leq|x-y|\right\}$, we have that

$$
G^{Y^{D}}(x, y) \geq c_{4} \frac{\Phi\left(\delta_{D}(x)\right)^{1 / 2}}{\Phi(|x-y|)^{1 / 2}} g(|x-y|) \text {. }
$$

Hence

$$
\mathbb{E}_{x} \tau_{D} \geq c_{5} \Phi\left(\delta_{D}(x)\right)^{1 / 2} \int_{\mathcal{C} \cap\left\{y \in D: \delta_{D}(x) \leq|x-y|\right\}} \frac{1}{\Phi(|x-y|)^{1 / 2}|x-y|^{d} \Phi(|x-y|)^{-1 / 2}}
$$




$$
\geq c_{6} \Phi\left(\delta_{D}(x)\right)^{1 / 2} \int_{\delta_{D}(x)}^{L} \frac{d s}{s} \geq c_{7} \Phi\left(\delta_{D}(x)\right)^{1 / 2} \log \left(1 / \delta_{D}(x)\right) .
$$

\section{Boundary Harnack principle in $C^{1,1}$ open set}

In this section we assume that the lower weak scaling index $\gamma_{1}$ of $\psi$ is strictly larger than $1 / 2$. We continue assuming that $d \geq 2$ and that $D \subset \mathbb{R}^{d}$ is a bounded $C^{1,1}$ open set. Again, let $(R, \Lambda)$ be the $C^{1,1}$ characteristics of $D$. Without loss of generality we assume that $R \leq 1$.

Combining (2.20) and (6.2)-(6.3), we immediately get the following

Proposition 7.1 For any $T>0$, there exists $C \geq 1$ such that for all $x, y \in D$,

$$
C^{-1} J^{D}(x, y) \leq J^{Y^{D}}(x, y) \leq C J^{D}(x, y)
$$

where

$$
\begin{aligned}
J^{D}(x, y):= & \int_{0}^{T}\left(\frac{\Phi\left(\delta_{D}(x)\right)^{1 / 2}}{s^{1 / 2}} \wedge 1\right)\left(\frac{\Phi\left(\delta_{D}(y)\right)^{1 / 2}}{s^{1 / 2}} \wedge 1\right) \\
& \times\left(\Phi^{-1}(s)^{-d} \wedge \frac{s}{|x-y|^{d} \Phi(|x-y|)}\right) \nu(s) d s+\Phi\left(\delta_{D}(x)\right)^{1 / 2} \Phi\left(\delta_{D}(y)\right)^{1 / 2} .
\end{aligned}
$$

Recall $\kappa_{0}=\left(1+(1+\Lambda)^{2}\right)^{-1 / 2}$, and for $Q \in \partial D, V_{Q}(r)$ is a $C^{1,1}$ domain with characteristic $(r R / L, \Lambda L / r)$ such that $D_{Q}(r / 2, r / 2) \subset V_{Q}(r) \subset D_{Q}(r, r)$ where $L=L(R, \Lambda, d)>0$. Recall that $g$ is defined in (5.2).

Lemma 7.2 There exists $C>0$ such that for every $r \leq \kappa_{0} R / 2, Q \in \partial D$ and $x \in$ $D_{Q}(r / 4, r / 4)$,

$$
\mathbb{E}_{x} \tau_{V_{Q}(r)} \leq \int_{V_{Q}(r)} G^{Y^{D}}(x, y) d y \leq C \frac{\Phi\left(\delta_{D}(x)\right)^{1 / 2}}{\Phi(r)^{1 / 2} \psi\left(\Phi(r)^{-1}\right)} .
$$

Proof. By using Theorem 6.4 in the second inequality, (6.12) in the third inequality, (2.2) in the penultimate inequality, and (2.1) and $\gamma_{1}>1 / 2$ in the last inequality, we get that

$$
\begin{aligned}
& \mathbb{E}_{x} \tau_{V_{Q}(r)}=\int_{V_{Q}(r)} G_{\tau_{V_{Q}(r)}}^{Y^{D}}(x, y) d y \leq \int_{V_{Q}(r)} G^{Y^{D}}(x, y) d y \\
& \leq c_{1} \Phi\left(\delta_{D}(x)\right)^{1 / 2} \int_{V_{Q}(r)} \frac{1}{\Phi(|x-y|)^{1 / 2}|x-y|^{d} \psi\left(\Phi(|x-y|)^{-1}\right)} d y \\
& \leq c_{1} \Phi\left(\delta_{D}(x)\right)^{1 / 2} \int_{B\left(x, 2 r / \kappa_{0}\right)} \frac{1}{\Phi(|x-y|)^{1 / 2}|x-y|^{d} \psi\left(\Phi(|x-y|)^{-1}\right)} d y \\
& \leq c_{2} \Phi\left(\delta_{D}(x)\right)^{1 / 2} \int_{0}^{2 r / \kappa_{0}} \frac{1}{\Phi(s)^{1 / 2} s \psi\left(\Phi(s)^{-1}\right)} d s \\
& \leq c_{3} \frac{\Phi\left(\delta_{D}(x)\right)^{1 / 2}}{\Phi(r)^{1 / 2} \psi\left(\Phi(r)^{-1}\right)} \int_{0}^{2 r / \kappa_{0}}\left(\frac{\Phi(r)}{\Phi(s)}\right)^{1 / 2-\gamma_{1}} \frac{d s}{s}
\end{aligned}
$$




$$
\leq c_{4} \frac{\Phi\left(\delta_{D}(x)\right)^{1 / 2}}{\Phi(r)^{1 / 2} \psi\left(\Phi(r)^{-1}\right)} .
$$

Lemma 7.3 There exists $C>0$ such that for all $r \leq \kappa_{0} R / 2, Q \in D$ and $x \in D_{Q}\left(2^{-3} r, 2^{-3} r\right)$,

$$
\mathbb{P}_{x}\left(Y^{D}\left(\tau_{V_{Q}(r)}\right) \in D_{Q}(2 r, r) \backslash D_{Q}(3 r / 2, r)\right) \geq C \frac{\Phi\left(\delta_{D}(x)\right)^{1 / 2}}{\Phi(r)^{1 / 2}}
$$

Proof. Without loss of generality, we assume $Q=0$. Recall that $V_{0}(r) \subset D_{0}(r, r) \subset$ $D \cap B(0, r / \kappa)$. Note that, for $y \in D_{0}(2 r, r) \backslash D_{0}(3 r / 2, r)$ and $z \in V_{0}(r)$, it holds that

$$
|z-y| \asymp^{c_{1}} r, \quad \delta_{D}(y) \geq c_{2} r \quad \text { and } \quad \delta_{D}(z) \leq c_{3} r .
$$

Using this and (2.9), (2.5) and Proposition 7.1, we have that for $z \in V_{0}(r)$,

$$
\begin{aligned}
& \int_{D_{0}(2 r, r) \backslash D_{0}(3 r / 2, r)} J^{Y^{D}}(z, y) d y \\
& \geq c_{4} \int_{D_{0}(2 r, r) \backslash D_{0}(3 r / 2, r)} \int_{\Phi(|y-z|)}^{2 \Phi(|y-z|)}\left(\frac{\Phi\left(\delta_{D}(z)\right)^{1 / 2}}{t^{1 / 2}} \wedge 1\right)\left(\frac{\Phi\left(\delta_{D}(y)\right)^{1 / 2}}{t^{1 / 2}} \wedge 1\right) \Phi^{-1}(t)^{-d} \nu(t) d t d y \\
& \asymp^{c_{5}} \int_{D_{0}(2 r, r) \backslash D_{0}(3 r / 2, r)}\left(\frac{\Phi\left(\delta_{D}(z)\right)^{1 / 2}}{\Phi(|z-y|)^{1 / 2}} \wedge 1\right) \frac{\psi\left(\Phi(|z-y|)^{-1}\right)}{|z-y|^{d}} d y \\
& \geq c_{6} \frac{\Phi\left(\delta_{D}(z)\right)^{1 / 2}}{\Phi(r)^{1 / 2}} \psi\left(\Phi(r)^{-1}\right) .
\end{aligned}
$$

For $a, b>0$, we define the cone $\mathcal{C}(x, a, b)$ above $x$ by

$$
\mathcal{C}(x, a, b):=\left\{y=\left(\widetilde{y}, y_{d}\right) \in B(x, a) \text { in } C S: y_{d}>x_{d},|\widetilde{x}-\widetilde{y}|<b\left(y_{d}-x_{d}\right)\right\} .
$$

Since $D$ is $C^{1,1}$ and $x \in D_{0}\left(2^{-3} r, 2^{-3} r\right)$, it is easy to see that there exists $\varepsilon \in\left(0,(2(1+\Lambda))^{-2}\right)$ such that

$$
\mathcal{C}\left(x, 2^{-4} r, \varepsilon\right) \subset D_{0}\left(2^{-2} r, 2^{-2} r\right)
$$

By (17.3) and the fact that $D_{0}(r / 2, r / 2) \subset V_{0}(r) \subset D_{0}(r, r)$, we have that $\delta_{D}(z)=\delta_{V_{0}(r)}(z)$ for all $z \in \overline{\mathcal{C}\left(x, 2^{-4} r, \varepsilon\right)}$.

It is easy to see that there exists $c_{5} \in(0,1]$ such that

$$
c_{5}|x-z| \leq \delta_{V_{0}(r)}(z)=\delta_{D}(z), \quad z \in \overline{\mathcal{C}\left(x, 2^{-5} r, 2^{-1} \varepsilon\right)} .
$$

We claim that for $z \in \mathcal{C}\left(x, 2^{-5} r, 2^{-1} \varepsilon\right)$,

$$
\left(\frac{\Phi\left(\delta_{D}(x)\right)}{\Phi(|x-z|)} \wedge 1\right) \Phi\left(\delta_{D}(z)\right) \geq c_{6} \Phi\left(\delta_{D}(x)\right) .
$$


If $z \in \mathcal{C}\left(x, 2^{-5} r, 2^{-1} \varepsilon\right) \backslash B\left(x, \delta_{D}(x) / 2\right)$, then $|x-z| \geq \delta_{D}(x) / 2$, so by (17.4) and (2.9),

$$
\left(\frac{\Phi\left(\delta_{D}(x)\right)}{\Phi(|x-z|)} \wedge 1\right) \Phi\left(\delta_{D}(z)\right) \geq c_{7}\left(\frac{\Phi\left(\delta_{D}(x)\right)}{\Phi(|x-z|)} \wedge 1\right) \Phi(|x-z|) \geq c_{8} \Phi\left(\delta_{D}(x)\right) .
$$

If $z \in \mathcal{C}\left(x, 2^{-5} r, 2^{-1} \varepsilon\right) \cap B\left(x, \delta_{D}(x) / 2\right)$ then $|x-z|<\delta_{D}(x) / 2$ and so by (2.9),

$$
\Phi\left(\delta_{D}(z)\right) \geq \Phi\left(\delta_{D}(x)-|x-z|\right)>\Phi\left(\delta_{D}(x) / 2\right) \geq 2^{-2} \Phi\left(\delta_{D}(x)\right) .
$$

Thus,

$$
\left(\frac{\Phi\left(\delta_{D}(x)\right)}{\Phi(|x-z|)} \wedge 1\right) \Phi\left(\delta_{D}(z)\right) \geq \Phi\left(\delta_{D}(z)\right) \geq 2^{-2} \Phi\left(\delta_{D}(x)\right) .
$$

We have proved (7.5)

Since $G_{V_{0}(r)}^{Y^{D}} \geq G^{Y^{V_{0}(r)}}$ by (2.27), we have by using Proposition 6.5 in the second below, (7.4) in the third, (7.5) in the fourth and (2.3) in the fifth,

$$
\begin{aligned}
& \mathbb{E}_{x} \int_{0}^{\tau_{V_{0}(r)}} \Phi\left(\delta_{D}\left(Y_{t}^{D}\right)\right)^{1 / 2} d t=\int_{V_{0}(r)} G_{V_{0}(r)}^{Y^{D}}(x, z) \Phi\left(\delta_{D}(z)\right)^{1 / 2} d z \\
& \geq c_{9} \int_{V_{0}(r)}\left(\frac{\Phi\left(\delta_{V_{0}(r)}(x)\right)^{1 / 2}}{\Phi(|x-z|)^{1 / 2}} \wedge 1\right)\left(\frac{\Phi\left(\delta_{V_{0}(r)}(z)\right)^{1 / 2}}{\Phi(|x-z|)^{1 / 2}} \wedge 1\right) g(|x-z|) \Phi\left(\delta_{D}(z)\right)^{1 / 2} d z \\
& \geq c_{10} \int_{\mathcal{C}\left(x, 2^{-5} r, \varepsilon / 2\right)}\left(\frac{\Phi\left(\delta_{D}(x)\right)^{1 / 2}}{\Phi(|x-z|)^{1 / 2}} \wedge 1\right) g(|x-z|) \Phi\left(\delta_{D}(z)\right)^{1 / 2} d z \\
& \geq c_{11} \Phi\left(\delta_{D}(x)\right)^{1 / 2} \int_{\mathcal{C}\left(x, 2^{-5} r, \varepsilon / 2\right)} g(|x-z|) d z \\
& \geq c_{12} \Phi\left(\delta_{D}(x)\right)^{1 / 2} \int_{0}^{2^{-5} r} \frac{1}{s \psi\left(\Phi(s)^{-1}\right)} d s \geq c_{13} \frac{\Phi\left(\delta_{D}(x)\right)^{1 / 2}}{\psi\left(\Phi(r)^{-1}\right)} .
\end{aligned}
$$

Combining (7.2) and (7.6), we get

$$
\begin{aligned}
& \mathbb{P}_{x}\left(Y^{D}\left(\tau_{V_{0}(r)}\right) \in D_{0}(2 r, r) \backslash D_{0}(3 r / 2, r)\right)=\mathbb{E}_{x} \int_{0}^{\tau_{V_{0}(r)}} \int_{D_{0}(2 r, r) \backslash D_{0}(3 r / 2, r)} J^{Y^{D}}\left(Y_{t}^{D}, y\right) d y d t \\
& \geq c_{14} \frac{1}{\Phi(r)^{1 / 2}} \psi\left(\Phi(r)^{-1}\right) \mathbb{E}_{x} \int_{0}^{\tau_{V_{0}(r)}} \Phi\left(\delta_{D}\left(Y_{t}^{D}\right)\right)^{1 / 2} d t \\
& \geq c_{15} \frac{1}{\Phi(r)^{1 / 2}} \psi\left(\Phi(r)^{-1}\right) \frac{\Phi\left(\delta_{D}(x)\right)^{1 / 2}}{\psi\left(\Phi(r)^{-1}\right)}=c_{15} \frac{\Phi\left(\delta_{D}(x)\right)^{1 / 2}}{\Phi(r)^{1 / 2}} .
\end{aligned}
$$

Let

$$
j(r):=\frac{\psi\left(\Phi(r)^{-1}\right)}{r^{d}}, \quad r>0 .
$$

Note that $j$ is a decreasing function and it satisfies the doubling property near 0 . 
Lemma 7.4 Assume that $\gamma_{1}>1 / 2$. There exists $C>0$ such that for every $r \leq \kappa_{0} R / 2$, $Q \in \partial D$ and $x \in D_{Q}\left(2^{-3} r, 2^{-4} r\right)$,

$$
\mathbb{P}_{x}\left(Y^{D}\left(\tau_{V_{Q}(r)}\right) \in D_{Q}(2 r, 2 r)\right) \leq C \frac{\Phi\left(\delta_{D}(x)\right)^{1 / 2}}{\Phi(r)^{1 / 2}}
$$

Proof. Without loss of generality, we assume $Q=0$ and fix $r \leq \kappa^{-1} R / 2$. Note that, by (6.12), $V_{0}(r) \subset D_{0}(r, r) \subset D \cap B\left(0, r / \kappa_{0}\right)$. Using the Lévy system formula and (2.22), (2.24), (2.27) we get

$$
\begin{aligned}
& \mathbb{P}_{x}\left(Y^{D}\left(\tau_{V_{0}(r)}\right) \in D_{0}(2 r, r) \backslash D_{0}(3 r / 2, r)\right) \\
= & \mathbb{E}_{x} \int_{0}^{\tau_{V_{0}}(r)} \int_{D_{0}(2 r, r) \backslash D_{0}(3 r / 2, r)} J^{Y^{D}}\left(Y_{t}^{D}, z\right) d z d t \\
= & \int_{V_{0}(r)} G_{V_{0}(r)}^{Y^{D}}(x, y) \int_{D_{0}(2 r, r) \backslash D_{0}(3 r / 2, r)} J^{Y^{D}}(y, z) d z d y \\
\leq & \int_{D_{0}(2 r, r) \backslash D_{0}(3 r / 2, r)} \int_{V_{0}(r)} G^{Y^{D}}(x, y) J^{X}(y, z) d y d z .
\end{aligned}
$$

We first note that

$$
c_{0} r<|y-z| \leq 4\left(1+\kappa_{0}^{-1}\right) r \quad \text { for }(y, z) \in\left(V_{0}(r) \times\left(D_{0}(2 r, r) \backslash D_{0}(3 r / 2, r)\right) .\right.
$$

Thus by (2.23) and Lemma 7.2 , for $x \in D_{0}(r / 4, r / 4)$ and $z \in D_{0}(2 r, r) \backslash D_{0}(3 r / 2, r)$,

$$
\begin{aligned}
& \int_{V_{0}(r)} G^{Y^{D}}(x, y) J^{X}(y, z) d y \\
& \leq c_{1} \int_{V_{0}(r)} G^{Y^{D}}(x, y) j(|y-z|) d y \\
& \leq c_{2} \int_{V_{0}(r)} G^{Y^{D}}(x, y) d y \frac{\psi\left(\Phi(r)^{-1}\right)}{r^{d}} \leq c_{3} \frac{\Phi\left(\delta_{D}(x)\right)^{1 / 2}}{\Phi(r)^{1 / 2}} r^{-d} .
\end{aligned}
$$

By (7.9) and (7.11) we obtain that for $x \in D_{0}(r / 4, r / 4)$

$$
\mathbb{P}_{x}\left(Y^{D}\left(\tau_{V_{0}(r)}\right) \in D_{0}(2 r, r) \backslash D_{0}(3 r / 2, r)\right) \leq c_{4} \frac{\Phi\left(\delta_{D}(x)\right)^{1 / 2}}{\Phi(r)^{1 / 2}}
$$

Note that, by the same argument in the proof of Lemma 7.2 , we get that for $w \in D_{0}(r / 2, r / 2)$ and each $\tilde{r} \in(0, r / 2)$,

$$
\int_{D \cap B(w, \tilde{r})} G^{Y^{D}}(w, z) d z \leq c_{5} \frac{\Phi\left(\delta_{D}(w)\right)^{1 / 2}}{\Phi(r)^{1 / 2} \psi\left(\Phi(\tilde{r})^{-1}\right)} .
$$

Thus, using (7.13), we have that for $w \in D_{0}(r / 2, r / 2)$ and $0<4 R_{1} \leq R_{2}<r$,

$$
\mathbb{P}_{w}\left(Y_{\tau_{D \cap B\left(w, R_{1}\right)}^{D}}^{D} \in D \backslash B\left(w, R_{2}\right)\right)
$$




$$
\begin{aligned}
& =\int_{D \cap B\left(w, R_{1}\right)} G_{D \cap B\left(w, R_{1}\right)}^{Y^{D}}(w, z) \int_{D \backslash B\left(w, R_{2}\right)} J^{Y^{D}}(y, z) d y d z \\
& \leq c_{6} \int_{D \cap B\left(w, R_{1}\right)} G^{Y^{D}}(w, z) \int_{\mathbb{R}^{d} \backslash B\left(0, R_{2}\right)}|y|^{-d} \psi\left(\Phi(|y|)^{-1}\right) d y d z \\
& \leq c_{7} \frac{\Phi\left(\delta_{D}(w)\right)^{1 / 2}}{\Phi\left(R_{1}\right)^{1 / 2} \psi\left(\Phi\left(R_{1}\right)^{-1}\right)} \int_{R_{2}}^{\infty} s^{-1} \psi\left(\Phi(s)^{-1}\right) d s \leq c_{8} \frac{\Phi\left(\delta_{D}(w)\right)^{1 / 2}}{\Phi\left(R_{1}\right)^{1 / 2} \psi\left(\Phi\left(R_{1}\right)^{-1}\right)} \psi\left(\Phi\left(R_{2}\right)^{-1}\right) .
\end{aligned}
$$

Let

$$
H_{2}=\left\{Y^{D}\left(\tau_{V_{0}(r)}\right) \in D_{0}(2 r, 2 r)\right\}, \quad H_{1}=\left\{Y^{D}\left(\tau_{V_{0}(r)}\right) \in D_{0}(2 r, r) \backslash D_{0}(3 r / 2, r)\right\} .
$$

We claim that $\mathbb{P}_{x}\left(H_{2}\right) \leq c_{9} \mathbb{P}_{x}\left(H_{1}\right)$ for all $r \leq \kappa^{-1} R / 2$ and $x \in D_{0}\left(2^{-3} r, 2^{-4} r\right)$. Combining this claim with (7.12), we arrive at the conclusion of the lemma:

$$
\mathbb{P}_{x}\left(Y^{D}\left(\tau_{V_{0}(r)}\right) \in D_{0}(2 r, 2 r)\right) \leq c_{9} \mathbb{P}_{x}\left(H_{1}\right) \leq c_{10} \frac{\Phi\left(\delta_{D}(x)\right)^{1 / 2}}{\Phi(r)^{1 / 2}}, \quad x \in D_{0}\left(2^{-3} r, 2^{-4} r\right) .
$$

Now we give the proof of the claim, which is inspired by the proof of [8, Lemma 5.3] (see also [18, Lemma 6.2]). Note that, by Lemma 7.3, for $w \in D_{0}(r / 8, r / 8)$,

$$
\mathbb{P}_{w}\left(H_{1}\right) \geq c_{11} \frac{\Phi\left(\delta_{D}(w)\right)^{1 / 2}}{\Phi(r)^{1 / 2}} .
$$

For $i \geq 1$, set

$$
J_{i}=D_{0}\left(2^{-i-2} r, s_{i}\right) \backslash D_{0}\left(2^{-i-3} r, s_{i}\right), \quad s_{i}=\frac{1}{4}\left(\frac{1}{2}-\frac{1}{50} \sum_{j=1}^{i} \frac{1}{j^{2}}\right) r,
$$

and $s_{0}=s_{1}$. Note that $r /(10)<s_{i}<r / 8$. Define for $i \geq 1$,

$$
d_{i}=d_{i}(r)=\sup _{z \in J_{i}} \frac{\mathbb{P}_{z}\left(H_{2}\right)}{\mathbb{P}_{z}\left(H_{1}\right)}, \quad \widetilde{J}_{i}=D_{0}\left(2^{-i-2} r, s_{i-1}\right), \quad \tau_{i}=\tau_{\widetilde{J_{i}}} .
$$

By (7.15), $\sup _{r \leq \kappa^{-1} R / 2} d_{i}(r)$ is finite for all $i \geq 1$. Repeating the argument leading to [18, (6.29)], we get that for $z \in J_{i}$ and $i \geq 2$,

$$
\mathbb{P}_{z}\left(H_{2}\right) \leq\left(\sup _{1 \leq k \leq i-1} d_{k}\right) \mathbb{P}_{z}\left(H_{1}\right)+\mathbb{P}_{z}\left(Y_{\tau_{i}}^{D} \in D_{0}(2 r, 2 r) \backslash \cup_{k=1}^{i-1} J_{k}\right)
$$

For $i \geq 2$, define $\sigma_{i, 0}=0, \sigma_{i, 1}=\inf \left\{t>0:\left|Y_{t}^{D}-Y_{0}^{D}\right| \geq 2^{-i-2} r\right\}$ and $\sigma_{i, m+1}=$ $\sigma_{i, m}+\sigma_{i, 1} \circ \theta_{\sigma_{i, m}}$ for $m \geq 1$. By Lemma [5.2, we have that there exists $k_{1} \in(0,1)$ such that

$$
\mathbb{P}_{w}\left(Y_{\sigma_{i, 1}}^{D} \in \widetilde{J}_{i}\right) \leq 1-\mathbb{P}_{w}\left(\sigma_{i, 1}=\zeta\right) \leq 1-\mathbb{P}_{w}\left(\tau_{B\left(w, \delta_{D}(w) / 2\right)}=\zeta\right)<k_{1}, \quad w \in \widetilde{J}_{i} .
$$

For the purpose of further estimates, we now choose a positive integer $l \geq b_{2} a_{2}^{\gamma_{2}}$ such that $k_{1}^{l} \leq 2^{-2 \gamma_{2} \delta_{2}}$, where $a_{2}, \delta_{2}$ and $b_{2}, \gamma_{2}$ are the constants from (2.1) and (2.2) respectively. Next 
we choose $i_{0} \geq 2$ large enough so that $2^{-i}<1 /\left(200 l i^{3}\right)$ for all $i \geq i_{0}$. Now we assume $i \geq i_{0}$. Using (7.18) and the strong Markov property we have that for $z \in J_{i}$,

$$
\begin{aligned}
& \mathbb{P}_{z}\left(\tau_{i}>\sigma_{i, l i}\right) \leq \mathbb{P}_{z}\left(Y_{\sigma_{i, k}}^{D} \in \widetilde{J}_{i}, 1 \leq k \leq l i\right) \\
& =\mathbb{E}_{z}\left[\mathbb{P}_{Y_{\sigma_{i, l i-1}}^{D}}\left(Y_{\sigma_{i, 1}}^{D} \in \widetilde{J}_{i}\right): Y_{\sigma_{i, l i-1}}^{D} \in \widetilde{J}_{i}, Y_{\sigma_{i, k}}^{D} \in \widetilde{J}_{i}, 1 \leq k \leq l i-2\right] \\
& \leq \mathbb{P}_{z}\left(Y_{\sigma_{i, k}}^{D} \in \widetilde{J}_{i}, 1 \leq k \leq l i-1\right) k_{1} \leq k_{1}^{l i} .
\end{aligned}
$$

Note that if $z \in J_{i}$ and $y \in D_{0}(2 r, 2 r) \backslash\left[\widetilde{J}_{i} \cup\left(\cup_{k=1}^{i-1} J_{k}\right)\right]$, then $|y-z| \geq\left(s_{i-1}-s_{i}\right) \wedge$ $\left(2^{-3}-2^{-i-2}\right) r=r /\left(200 i^{2}\right)$. Furthermore, since $2^{-i-2} r<r /\left(200 i^{2}\right)$ (recall that $\left.i \geq i_{0}\right)$, if $Y_{\tau_{i}}^{D}(\omega) \in D_{0}(2 r, 2 r) \backslash \cup_{k=1}^{i-1} J_{k}$ and $\tau_{i}(\omega) \leq \sigma_{i, l i}(\omega)$, then $\tau_{i}(\omega)=\sigma_{i, k}(\omega)$ for some $k=k(\omega) \leq l i$. Dependence of $k$ on $\omega$ will be omitted in the next few lines. Hence on $\left\{Y_{\tau_{i}}^{D} \in D_{0}(2 r, 2 r) \backslash\right.$ $\left.\cup_{k=1}^{i-1} J_{k}, \quad \tau_{i} \leq \sigma_{i, l i}\right\}$ with $Y_{0}^{D}=z \in J_{i}$, we have $\left|Y_{\sigma_{i, k}}^{D}-Y_{\sigma_{i, 0}}^{D}\right|=\left|Y_{\tau_{i}}^{D}-Y_{0}^{D}\right|>\frac{1}{200 i^{2}} r$ for some $1 \leq k \leq l i$. Thus for some $1 \leq k \leq l i$,

$$
\sum_{j=0}^{k}\left|Y_{\sigma_{i, j}}^{D}-Y_{\sigma_{i, j-1}}^{D}\right|>\frac{1}{200 i^{2}} r
$$

which implies for some $1 \leq j \leq k \leq l i$,

$$
\left|Y_{\sigma_{i, j}}^{D}-Y_{\sigma_{i, j-1}}^{D}\right| \geq \frac{1}{k} \frac{1}{200 i^{2}} r \geq \frac{1}{l i} \frac{1}{200 i^{2}} r .
$$

Thus, we have

$$
\begin{gathered}
\left\{Y_{\tau_{i}}^{D} \in D_{0}(2 r, 2 r) \backslash \cup_{k=1}^{i-1} J_{k}, \quad \tau_{i} \leq \sigma_{i, l i}\right\} \\
\subset \cup_{j=1}^{l i}\left\{\left|Y_{\sigma_{i, j}}^{D}-Y_{\sigma_{i, j-1}}^{D}\right| \geq r /\left(400 l i^{3}\right), Y_{\sigma_{i, j-1}}^{D} \in \widetilde{J}_{i}\right\} .
\end{gathered}
$$

Now, using (7.14) (noting that $4 \cdot 2^{-i-2}<1 /\left(400 l i^{3}\right)$ for all $\left.i \geq i_{0}\right)$, and repeating the argument leading to [18, (6.34)], we get

$$
\begin{aligned}
& \mathbb{P}_{z}\left(Y_{\tau_{i}}^{D} \in D_{0}(2 r, 2 r) \backslash \cup_{k=1}^{i-1} J_{k}, \quad \tau_{i} \leq \sigma_{i, l i}\right) \\
\leq & \operatorname{li} \sup _{z \in \widetilde{J}_{i}} \mathbb{P}_{z}\left(\left|Y_{\sigma_{i, 1}}^{D}-z\right| \geq r /\left(400 l i^{3}\right)\right) \leq c_{12} l i \frac{\psi\left(\Phi\left(r /\left(l i^{3}\right)\right)^{-1}\right)}{\psi\left(\Phi\left(2^{-i} r\right)^{-1}\right)} .
\end{aligned}
$$

By (7.19) and (7.21), we have for $z \in J_{i}$,

$$
\mathbb{P}_{z}\left(Y_{\tau_{i}}^{D} \in D_{0}(2 r, 2 r) \backslash \cup_{k=1}^{i-1} J_{k}\right) \leq k_{1}^{l i}+c_{12} l i \frac{\psi\left(\Phi\left(r /\left(l i^{3}\right)\right)^{-1}\right)}{\psi\left(\Phi\left(2^{-i} r\right)^{-1}\right)}
$$

By our choice of $l$, using (2.3) we have

$$
l i \frac{\psi\left(\Phi\left(r /\left(l i^{3}\right)\right)^{-1}\right)}{\psi\left(\Phi\left(2^{-i} r\right)^{-1}\right)} \geq a_{2}^{-\gamma_{2}} b_{2}^{-1} l i\left(l i^{3} / 2^{i}\right)^{2 \gamma_{2} \delta_{2}}=a_{2}^{-\gamma_{2}} b_{2}^{-1} l^{1+2 \gamma_{2} \delta_{2}} i^{1+6 \gamma_{2} \delta_{2}}\left(2^{-2 \gamma_{2} \delta_{2}}\right)^{i} \geq\left(k_{1}^{l}\right)^{i}
$$


Thus combining (7.23) with (7.22), and then using (7.15), (2.1), (2.2) and the fact that $\gamma_{1}>1 / 2$, we get that for $z \in J_{i}$,

$$
\begin{aligned}
& \frac{\mathbb{P}_{z}\left(Y_{\tau_{i}}^{D} \in D_{0}(2 r, 2 r) \backslash \cup_{k=1}^{i-1} J_{k}\right)}{\mathbb{P}_{z}\left(H_{1}\right)} \leq c_{13} l i \frac{\Phi(r)^{1 / 2}}{\Phi\left(2^{-i} r\right)^{1 / 2}} \frac{\psi\left(\Phi\left(r /\left(l i^{3}\right)\right)^{-1}\right)}{\psi\left(\Phi\left(2^{-i} r\right)^{-1}\right)} \\
& \leq c_{14} i \frac{\Phi(r)^{1 / 2}}{\Phi\left(2^{-i} r\right)^{1 / 2}} \frac{\Phi\left(2^{-i} r\right)^{\gamma_{1}}}{\Phi\left(r /\left(l i^{3}\right)\right)^{\gamma_{1}}}=c_{14} i \frac{\Phi(r)^{1 / 2}}{\Phi\left(r /\left(l i^{3}\right)\right)^{1 / 2}} \frac{\Phi\left(2^{-i} r\right)^{\gamma_{1}-1 / 2}}{\Phi\left(r /\left(l i^{3}\right)\right)^{\gamma_{1}-1 / 2}} \\
& \leq c_{15} i\left(l i^{3}\right)^{\delta_{2}}\left(2^{-i} l i^{3}\right)^{\left(2 \gamma_{1}-1\right) \delta_{1}}=c_{16} i^{1+3 \delta_{2}+3\left(2 \gamma_{1}-1\right) \delta_{1}} 2^{-i\left(2 \gamma_{1}-1\right) \delta_{1}} .
\end{aligned}
$$

By this and (17.17), for $z \in J_{i}$,

$$
\begin{aligned}
& \frac{\mathbb{P}_{z}\left(H_{2}\right)}{\mathbb{P}_{z}\left(H_{1}\right)} \leq \sup _{1 \leq k \leq i-1} d_{k}+\frac{\mathbb{P}_{z}\left(Y_{\tau_{i}}^{D} \in D_{0}(2 r, 2 r) \backslash \cup_{k=1}^{i-1} J_{k}\right)}{\mathbb{P}_{z}\left(H_{1}\right)} \\
\leq & \sup _{1 \leq k \leq i-1} d_{k}+c_{16} \frac{i^{1+3 \delta_{2}+3\left(2 \gamma_{1}-1\right) \delta_{1}}}{2^{i\left(2 \gamma_{1}-1\right) \delta_{1}}} \\
\leq & \sup _{1 \leq k \leq i-1} d_{k}+c_{16} \frac{i^{10}}{2^{i\left(2 \gamma_{1}-1\right) \delta_{1}}} .
\end{aligned}
$$

This implies that

$$
d_{i} \leq \sup _{1 \leq k \leq i_{0}-1} d_{k}+c_{16} \sum_{k=1}^{i} \frac{k^{10}}{2^{k\left(2 \gamma_{1}-1\right) \delta_{1}}} \leq \sup _{\substack{1 \leq k \leq i_{0}-1 \\ r \leq \kappa-1}} d_{k}(r)+c_{16} \sum_{k=1}^{\infty} \frac{k^{10}}{2^{k\left(2 \gamma_{1}-1\right) \delta_{1}}}=: c_{17}<\infty .
$$

Thus the claim above is valid, since $D_{0}\left(2^{-3} r, 2^{-4} r\right) \subset \cup_{k=1}^{\infty} J_{k}$. The proof is now complete.

We are now ready to prove the boundary Harnack principle with explicit decay rate near the boundary of $D$ when the lower weak scaling index $\gamma_{1}$ of $\psi$ is strictly larger than $1 / 2$.

Theorem 7.5 Assume that $\gamma_{1}>1 / 2$. Let $D \subset \mathbb{R}^{d}$ be a bounded $C^{1,1}$ open set with $C^{1,1}$ characteristics $(R, \Lambda)$. There exists a constant $C=C(d, \Lambda, R, \phi, \psi)>0$ such that for any $r \in(0, R], Q \in \partial D$, and any non-negative function $f$ in $D$ which is harmonic in $D \cap B(Q, r)$ with respect to $Y^{D}$ and vanishes continuously on $\partial D \cap B(Q, r)$, we have

$$
\frac{f(x)}{\Phi\left(\delta_{D}(x)\right)^{1 / 2}} \leq C \frac{f(y)}{\Phi\left(\delta_{D}(y)\right)^{1 / 2}} \quad \text { for all } x, y \in D \cap B(Q, r / 2) .
$$

Proof. In this proof, the constants $\eta$ and $c_{i}$ are always independent of $r$.

Note that, since $D$ is a $C^{1,1}$ open set and $r<R$, by Theorem 4.8, it suffices to prove (7.25) for $x, y \in D \cap B\left(Q, 2^{-7} \kappa_{0} r\right)$. Throughout the remainder of the proof we assume that $x \in D \cap B\left(Q, 2^{-7} \kappa_{0} r\right)$.

Let $Q_{x}$ be the point $Q_{x} \in \partial D$ so that $\left|x-Q_{x}\right|=\delta_{D}(x)$ and let $x_{0}:=Q_{x}+\frac{r}{8}\left(x-Q_{x}\right) / \mid x-$ $Q_{x} \mid$. We choose a $C^{1,1}$ function $\varphi: \mathbb{R}^{d-1} \rightarrow \mathbb{R}$ satisfying $\varphi(\widetilde{0})=0, \nabla \varphi(\widetilde{0})=(0, \ldots, 0)$, $\|\nabla \varphi\|_{\infty} \leq \Lambda,|\nabla \varphi(\widetilde{y})-\nabla \varphi(\widetilde{z})| \leq \Lambda|\widetilde{y}-\widetilde{z}|$, and an orthonormal coordinate system $C S$ with its origin at $Q_{x}$ such that

$$
B\left(Q_{x}, R\right) \cap D=\left\{y=\left(\widetilde{y}, y_{d}\right) \in B(0, R) \text { in } C S: y_{d}>\varphi(\widetilde{y})\right\} .
$$


In the coordinate system $C S$ we have $\widetilde{x}=\widetilde{0}$ and $x_{0}=(\widetilde{0}, r / 8)$. For any $b_{1}, b_{2}>0$, we define

$$
\widehat{D}\left(b_{1}, b_{2}\right):=\left\{y=\left(\widetilde{y}, y_{d}\right) \text { in } C S: 0<y_{d}-\varphi(\widetilde{y})<2^{-2} \kappa_{0} r b_{1},|\widetilde{y}|<2^{-2} \kappa_{0} r b_{2}\right\} .
$$

By (6.12), we have that $\widehat{D}(2,2) \subset D \cap B\left(Q_{x}, r / 2\right) \subset D \cap B(Q, r)$. Thus, since $f$ is harmonic in $D \cap B(Q, r)$ and vanishes continuously in $\partial D \cap B(Q, r)$, by Lemma 5.1, $f$ is regular harmonic in $\widehat{D}(2,2)$ and vanishes continuously in $\partial D \cap \widehat{D}(2,2)$.

Recall that $V(1):=V_{Q_{x}}\left(2^{-2} \kappa_{0} r\right)$ is a $C^{1,1}$ domain with $C^{1,1}$ characteristics $(r R / L, \Lambda L / r)$ such that $\widehat{D}(1 / 2,1 / 2) \subset V(1) \subset \widehat{D}(1,1)$, where $L=L(R, \Lambda, d)>0$.

By Theorem 4.7 (or Theorem 4.8) and Lemma 7.3, we have

$$
\begin{aligned}
& f(x)=\mathbb{E}_{x}\left[f\left(Y^{D}\left(\tau_{V(1)}\right)\right)\right] \geq \mathbb{E}_{x}\left[f\left(Y^{D}\left(\tau_{V(1)}\right)\right) ; Y_{\tau_{V(1)}}^{D} \in \widehat{D}(2,1) \backslash \widehat{D}(3 / 2,1)\right] \\
& \geq c_{16} f\left(x_{0}\right) \mathbb{P}_{x}\left(Y^{D}\left(\tau_{V(1)}\right) \in \widehat{D}(2,1) \backslash \widehat{D}(3 / 2,1)\right) \geq c_{17} \frac{\Phi\left(\delta_{D}(x)\right)^{1 / 2}}{\Phi(r)^{1 / 2}} f\left(x_{0}\right) .
\end{aligned}
$$

Take $w=\left(\widetilde{0}, 2^{-6} \kappa_{0} r\right)$. Then there exists $\epsilon \in(0,1 / 8)$ such that

$$
B\left(w, \epsilon 2^{-5} \kappa_{0} r\right) \subset \widehat{D}(1 / 2,1 / 2) \subset V(1) .
$$

Hence

$$
\begin{aligned}
& f(w) \geq \mathbb{E}_{w}\left[f\left(Y^{D}\left(\tau_{V(1)}\right)\right) ; Y^{D}\left(\tau_{V(1)}\right) \notin \widehat{D}(2,2)\right] \\
& =\mathbb{E}_{w} \int_{0}^{\tau_{V(1)}} \int_{D \backslash \widehat{D}(2,2)} J^{Y^{D}}\left(Y_{t}^{D}, y\right) f(y) d y d t \\
& \geq \mathbb{E}_{w} \int_{0}^{\tau_{B\left(w, \epsilon 2^{-5} \kappa_{0} r\right)}} \int_{D \backslash \widehat{D}(2,2)} J^{Y^{D}}\left(Y_{t}^{D}, y\right) f(y) d y d t \\
& \geq c_{18} \mathbb{E}_{w} \tau_{B\left(w, \epsilon 2^{-5} \kappa_{0} r\right)} \int_{D \backslash \widehat{D}(2,2)} J^{Y^{D}}(w, y) f(y) d y \\
& \geq c_{19} \frac{1}{\psi\left(\Phi(r)^{-1}\right)} \int_{D \backslash \widehat{D}(2,2)} J^{Y^{D}}(w, y) f(y) d y
\end{aligned}
$$

where in the fourth line we used Proposition 4.5, and in the last line we used Lemma 4.6 and (2.3).

Further, note that for any $z \in V(1)$ and $y \in D \backslash \widehat{D}(2,2)$ we have that $\delta_{D}(z) \leq c_{23} r \leq$ $c_{24} \delta_{D}(w)$ and $|z-y| \asymp^{c_{25}}|w-y|$. By using these two observations and Proposition 7.1 we see that

$$
\begin{aligned}
& J^{Y^{D}}(z, y) \leq c_{26}\left(\int_{0}^{1}\left(\frac{\Phi\left(\delta_{D}(z)\right)^{1 / 2}}{s^{1 / 2}} \wedge 1\right)\left(\frac{\Phi\left(\delta_{D}(y)\right)^{1 / 2}}{s^{1 / 2}} \wedge 1\right)\right. \\
& \left.\quad \times\left(\Phi^{-1}(s)^{-d} \wedge \frac{s}{|z-y|^{d} \Phi(|z-y|)}\right) \nu(s) d s+\Phi\left(\delta_{D}(z)\right)^{1 / 2} \Phi\left(\delta_{D}(y)\right)^{1 / 2}\right) \\
& \leq c_{27}\left(\int_{0}^{1}\left(\frac{\Phi\left(\delta_{D}(w)\right)^{1 / 2}}{s^{1 / 2}} \wedge 1\right)\left(\frac{\Phi\left(\delta_{D}(y)\right)^{1 / 2}}{s^{1 / 2}} \wedge 1\right)\right.
\end{aligned}
$$




$$
\begin{aligned}
& \left.\times\left(\Phi^{-1}(s)^{-d} \wedge \frac{s}{|w-y|^{d} \Phi(|w-y|)}\right) \nu(s) d s+\Phi\left(\delta_{D}(w)\right)^{1 / 2} \Phi\left(\delta_{D}(y)\right)^{1 / 2}\right) \\
\leq & c_{28} J^{Y^{D}}(w, y) .
\end{aligned}
$$

Hence, combining Lemma 7.2 with (7.27)-(7.28) we now have

$$
\begin{aligned}
& \mathbb{E}_{x}\left[f\left(Y^{D}\left(\tau_{V(1)}\right)\right) ; Y^{D}\left(\tau_{V(1)}\right) \notin \widehat{D}(2,2)\right] \\
& =\mathbb{E}_{x} \int_{0}^{\tau_{V(1)}} \int_{D \backslash \widehat{D}(2,2)} J^{Y^{D}}\left(Y_{t}^{D}, y\right) f(y) d y d t \\
& \leq c_{28} \mathbb{E}_{x} \tau_{V(1)} \int_{D \backslash \widehat{D}(2,2)} J^{Y^{D}}(w, y) f(y) d y \\
& \leq c_{29} \frac{\Phi\left(\delta_{D}(x)\right)^{1 / 2}}{\Phi(r)^{1 / 2} \psi\left(\Phi(r)^{-1}\right)} \int_{D \backslash \widehat{D}(2,2)} J^{Y^{D}}(w, y) f(y) d y \\
& \leq c_{30} \frac{\Phi\left(\delta_{D}(x)\right)^{1 / 2}}{\Phi(r)^{1 / 2}} f(w) .
\end{aligned}
$$

On the other hand, by Theorems 4.8, 5.5] and Lemma [7.4, we have

$$
\begin{aligned}
& \mathbb{E}_{x}\left[f\left(Y^{D}\left(\tau_{V(1)}\right)\right) ; Y^{D}\left(\tau_{V(1)}\right) \in \widehat{D}(2,2)\right] \\
& \leq c_{31} f\left(x_{0}\right) \mathbb{P}_{x}\left(Y^{D}\left(\tau_{V(1)}\right) \in \widehat{D}(2,2)\right) \leq c_{32} f\left(x_{0}\right) \frac{\Phi\left(\delta_{D}(x)\right)^{1 / 2}}{\Phi(r)^{1 / 2}} .
\end{aligned}
$$

Combining (7.29) and (17.30) and using Theorems 4.8 and 5.5 again, we get

$$
\begin{aligned}
& f(x)=\mathbb{E}_{x}\left[f\left(Y^{D}\left(\tau_{V(1)}\right)\right) ; Y^{D}\left(\tau_{V(1)}\right) \in \widehat{D}(2,2)\right]+\mathbb{E}_{x}\left[f\left(Y^{D}\left(\tau_{V(1)}\right)\right) ; Y^{D}\left(\tau_{V(1)}\right) \notin \widehat{D}(2,2)\right] \\
& \leq c_{33}\left(\frac{\Phi\left(\delta_{D}(x)\right)^{1 / 2}}{\Phi(r)^{1 / 2}} f\left(x_{0}\right)+\frac{\Phi\left(\delta_{D}(x)\right)^{1 / 2}}{\Phi(r)^{1 / 2}} f(w)\right) \leq c_{34} \frac{\Phi\left(\delta_{D}(x)\right)^{1 / 2}}{\Phi(r)^{1 / 2}} f\left(x_{0}\right) .
\end{aligned}
$$

Together with (7.26) we get that

$$
f(x) \asymp^{c_{35}} \frac{\Phi\left(\delta_{D}(x)\right)^{1 / 2}}{\Phi(r)^{1 / 2}} f\left(x_{0}\right)
$$

For any $y \in D \cap B\left(Q, 2^{-7} \kappa_{0} r\right)$, we have the same estimate with $f\left(y_{0}\right)$ instead of $f\left(x_{0}\right)$ where $y_{0}=Q_{y}+\frac{r}{8}\left(y-Q_{y}\right) /\left|y-Q_{y}\right|$ and $Q_{y} \in \partial D$ with $\left|y-Q_{y}\right|=\delta_{D}(y)$. Since $D$ is $C^{1,1}$, using Theorem 4.8, $f\left(y_{0}\right) \asymp^{c_{36}} f\left(x_{0}\right)$. It follows therefore from (7.32) that for every $x, y \in D \cap B\left(Q, 2^{-7} \kappa_{0} r\right)$,

$$
\frac{f(x)}{f(y)} \leq c_{37} \frac{\Phi\left(\delta_{D}(x)\right)^{1 / 2}}{\Phi\left(\delta_{D}(y)\right)^{1 / 2}}
$$

which proves the theorem. 


\section{Jumping kernel estimates}

In this section we continue assuming that $d \geq 2$ and that $D$ is a bounded $C^{1,1}$ open set in $\mathbb{R}^{d}$ with $C^{1,1}$ characteristics $(R, \Lambda)$.

The goal of the section is to derive sharp two-sided estimates for the jumping kernel $J^{Y^{D}}$. Somewhat surprisingly, obtaining explicit bounds not involving integral terms does not seem to be possible without additional assumptions on the Laplace exponent $\psi$. In case when $\psi(\lambda)=\lambda^{\gamma}$, this can be explained by the different qualitative boundary behaviors of $J^{Y^{D}}$ in cases $\gamma \in(0,1 / 2), \gamma=1 / 2$ and $\gamma \in(1 / 2,1)$, cf. Example 8.5.

Let

$$
\theta(t):=\Phi(t) \psi\left(\Phi(t)^{-1}\right) \quad \text { and } \quad \eta(t):=\Phi(t)^{1 / 2} \psi\left(\Phi(t)^{-1}\right), \quad t>0 .
$$

It follows that, as a composition of two increasing functions, cf. (2.11), $\theta(t)$ is an increasing function.

It is also straightforward to see that

$$
\left(1 \wedge \lambda^{2}\right) \theta(t) \leq \theta(\lambda t) \leq\left(1 \vee \lambda^{2}\right) \theta(t), \quad \lambda, t>0 .
$$

We will say that a function $f:(0, \infty) \rightarrow[0, \infty)$ is almost increasing near 0 if for every $T>0$ there exists a constant $C=C(T)>0$ such that $f(s) \leq C f(t)$ for all $0<s<t \leq T$. An almost decreasing function is defined analogously.

Recall that $r(t, x, y), g$ and $j$ are defined in (6.1), (5.2) and (7.7) respectively.

Lemma 8.1 (i) Suppose that $r \mapsto r^{1 / 2} \psi\left(r^{-1}\right)$ is almost decreasing near 0 and that for each $T>0$ there is a constant $C_{4}=C_{4}(T, \psi)>0$ such that

$$
\int_{r}^{T} s^{-1 / 2} \psi\left(s^{-1}\right) d s \leq C_{4} r^{1 / 2} \psi\left(r^{-1}\right) \quad \text { for } r \in(0, T] .
$$

Then there exists $C \geq 1$ such that for all $x, y \in D$,

$$
\int_{0}^{\Phi(|x-y|)} r(t, x, y) \nu(t) d t \asymp^{C} \frac{\psi\left(\Phi(|x-y|)^{-1}\right)}{|x-y|^{d}}\left(\frac{\theta\left(\delta_{D}(x) \wedge \delta_{D}(y)\right)}{\theta(|x-y|)} \wedge 1\right) .
$$

(ii) Suppose that $r \mapsto r^{1 / 2} \psi\left(r^{-1}\right)$ is almost increasing near 0 and that for every $T>0$, there is a constant $C_{5}=C_{5}(T, \psi)>0$ such that

$$
\int_{0}^{r} s^{-1 / 2} \psi\left(s^{-1}\right) d s \leq C_{5} r^{1 / 2} \psi\left(r^{-1}\right) \quad \text { for every } r \in(0, T] .
$$

Then there exists $C \geq 1$ such that for all $x, y \in D$,

$$
\begin{aligned}
& \int_{0}^{\Phi(|x-y|)} r(t, x, y) \nu(t) d t \\
& \asymp^{C} \frac{\psi\left(\Phi(|x-y|)^{-1}\right)}{|x-y|^{d}}\left(\frac{\Phi\left(\delta_{D}(x) \wedge \delta_{D}(y)\right)^{1 / 2}}{\Phi(|x-y|)^{1 / 2}} \wedge 1\right)\left(\frac{\eta\left(\delta_{D}(x) \vee \delta_{D}(y)\right)}{\eta(|x-y|)} \wedge 1\right) .
\end{aligned}
$$

Remark 8.2 It is easy to see that if $\psi$ satisfies (2.2) with $\gamma_{1}>1 / 2$, then the assumptions in Lemma 8.1(i) hold true. Similarly, if $\psi$ satisfies (2.2) with $\gamma_{2}<1 / 2$, then the assumptions in Lemma 8.1(ii) are true. 
Proof of Lemma 8.1 Put $T:=2 \Phi(\operatorname{diam}(D))$ By using (2.5), (6.1) and (4.4) we see that

$$
\begin{aligned}
& \int_{0}^{\Phi(|x-y|)} r(t, x, y) \nu(t) d t \\
& \quad \asymp \frac{1}{|x-y|^{d} \Phi(|x-y|)} \int_{0}^{\Phi(|x-y|)}\left(\frac{\Phi\left(\delta_{D}(x)\right)^{1 / 2}}{t^{1 / 2}} \wedge 1\right)\left(\frac{\Phi\left(\delta_{D}(y)\right)^{1 / 2}}{t^{1 / 2}} \wedge 1\right) \psi\left(t^{-1}\right) d t \\
& \quad=: \frac{1}{|x-y|^{d} \Phi(|x-y|)} I .
\end{aligned}
$$

Let

$$
\begin{gathered}
I_{1}:=\int_{0}^{\Phi\left(\delta_{D}(x)\right)} \psi\left(t^{-1}\right) d t, \quad I_{2}:=\Phi\left(\delta_{D}(x)\right)^{1 / 2} \int_{\Phi\left(\delta_{D}(x)\right)}^{\Phi\left(\delta_{D}(y)\right)} t^{-1 / 2} \psi\left(t^{-1}\right) d t \\
I_{3}:=\Phi\left(\delta_{D}(x)\right)^{1 / 2} \Phi\left(\delta_{D}(y)\right)^{1 / 2} \int_{\Phi\left(\delta_{D}(y)\right)}^{\Phi(|x-y|)} t^{-1} \psi\left(t^{-1}\right) d t
\end{gathered}
$$

and

$$
I_{4}:=\Phi\left(\delta_{D}(x)\right)^{1 / 2} \int_{\Phi\left(\delta_{D}(x)\right)}^{\Phi(|x-y|)} t^{-1 / 2} \psi\left(t^{-1}\right) d t
$$

Upper bound: Without loss of generality we assume $\delta_{D}(x) \leq \delta_{D}(y)$ and consider three cases:

(1) $\delta_{D}(x) \leq \delta_{D}(y) \leq|x-y|$ : Then $I=I_{1}+I_{2}+I_{3}$.

By (2.2), we have $I_{1} \asymp^{c_{1}} \psi\left(\Phi\left(\delta_{D}(x)\right)^{-1}\right) \Phi\left(\delta_{D}(x)\right)$ and

$$
I_{3} \leq c_{2} \Phi\left(\delta_{D}(x)\right)^{1 / 2} \Phi\left(\delta_{D}(y)\right)^{1 / 2} \psi\left(\Phi\left(\delta_{D}(y)\right)^{-1}\right) .
$$

In case (i), by (8.2),

$$
I_{2} \leq \Phi\left(\delta_{D}(x)\right)^{1 / 2} \int_{\Phi\left(\delta_{D}(x)\right)}^{2 T} t^{-1 / 2} \psi\left(t^{-1}\right) d t \leq c_{3} \psi\left(\Phi\left(\delta_{D}(x)\right)^{-1}\right) \Phi\left(\delta_{D}(x)\right) .
$$

By using that $r^{1 / 2} \psi\left(r^{-1}\right)$ is almost decreasing near 0 we see that

$$
\begin{aligned}
I & \leq c_{4}\left[\psi\left(\Phi\left(\delta_{D}(x)\right)^{-1}\right) \Phi\left(\delta_{D}(x)\right)+\Phi\left(\delta_{D}(x)\right)^{1 / 2} \Phi\left(\delta_{D}(y)\right)^{1 / 2} \psi\left(\Phi\left(\delta_{D}(y)\right)^{-1}\right)\right] \\
& \leq c_{5} \psi\left(\Phi\left(\delta_{D}(x)\right)^{-1}\right) \Phi\left(\delta_{D}(x)\right)=c_{5} \psi\left(\Phi\left(\delta_{D}(x) \wedge \delta_{D}(y)\right)^{-1}\right) \Phi\left(\delta_{D}(x) \wedge \delta_{D}(y)\right) .
\end{aligned}
$$

In case (ii), by (8.3), we have

$$
I_{2} \leq \Phi\left(\delta_{D}(x)\right)^{1 / 2} \int_{0}^{\Phi\left(\delta_{D}(y)\right)} t^{-1 / 2} \psi\left(t^{-1}\right) d t \leq c_{6} \Phi\left(\delta_{D}(x)\right)^{1 / 2} \Phi\left(\delta_{D}(y)\right)^{1 / 2} \psi\left(\Phi\left(\delta_{D}(y)\right)^{-1}\right) .
$$

By using that $r^{1 / 2} \psi\left(r^{-1}\right)$ is almost increasing near 0 we see that

$$
\begin{aligned}
I & \leq c_{7}\left[\psi\left(\Phi\left(\delta_{D}(x)\right)^{-1}\right) \Phi\left(\delta_{D}(x)\right)+\Phi\left(\delta_{D}(x)\right)^{1 / 2} \Phi\left(\delta_{D}(y)\right)^{1 / 2} \psi\left(\Phi\left(\delta_{D}(y)\right)^{-1}\right)\right] \\
& \leq c_{8} \Phi\left(\delta_{D}(x) \wedge \delta_{D}(y)\right)^{1 / 2} \Phi\left(\delta_{D}(x) \vee \delta_{D}(y)\right)^{1 / 2} \psi\left(\Phi\left(\delta_{D}(x) \vee \delta_{D}(y)\right)^{-1}\right) .
\end{aligned}
$$

(2) $\delta_{D}(x) \leq|x-y| \leq \delta_{D}(y)$ : Then $I=I_{1}+I_{4}$. 
In case (i), by (8.2), we have

$$
I_{4} \leq \Phi\left(\delta_{D}(x)\right)^{1 / 2} \int_{\Phi\left(\delta_{D}(x)\right)}^{2 T} t^{-1 / 2} \psi\left(t^{-1}\right) d t \leq c_{9} \psi\left(\Phi\left(\delta_{D}(x)\right)^{-1}\right) \Phi\left(\delta_{D}(x)\right) .
$$

Thus we see that

$$
I \leq c_{10} \psi\left(\Phi\left(\delta_{D}(x)\right)^{-1}\right) \Phi\left(\delta_{D}(x)\right)=c_{10} \psi\left(\Phi\left(\delta_{D}(x) \wedge \delta_{D}(y)\right)^{-1}\right) \Phi\left(\delta_{D}(x) \wedge \delta_{D}(y)\right) .
$$

In case (ii), by (8.3), we have

$$
I_{4} \leq \Phi\left(\delta_{D}(x)\right)^{1 / 2} \int_{0}^{\Phi(|x-y|)} t^{-1 / 2} \psi\left(t^{-1}\right) d t \leq c_{11} \Phi\left(\delta_{D}(x)\right)^{1 / 2} \Phi(|x-y|)^{1 / 2} \psi\left(\Phi(|x-y|)^{-1}\right) .
$$

By using that $r^{1 / 2} \psi\left(r^{-1}\right)$ is almost increasing near 0 , we see that

$$
\begin{aligned}
I & \leq c_{12}\left(\psi\left(\Phi\left(\delta_{D}(x)\right)^{-1}\right) \Phi\left(\delta_{D}(x)\right)+\Phi\left(\delta_{D}(x)\right)^{1 / 2} \Phi(|x-y|)^{1 / 2} \psi\left(\Phi(|x-y|)^{-1}\right)\right) \\
& \leq c_{13} \Phi\left(\delta_{D}(x)\right)^{1 / 2} \Phi(|x-y|)^{1 / 2} \psi\left(\Phi(|x-y|)^{-1}\right) .
\end{aligned}
$$

(3) $|x-y| \leq \delta_{D}(x) \leq \delta_{D}(y)$ : Then by (2.2),

$$
I=\int_{0}^{\Phi(|x-y|)} \psi\left(t^{-1}\right) d t \asymp^{c_{14}} \Phi(|x-y|) \psi\left(\Phi(|x-y|)^{-1}\right) .
$$

Lower bound: Again, we assume $\delta_{D}(x) \leq \delta_{D}(y)$. Let $M:=\left(2 / a_{1}\left(T^{-2}\right)\right)^{1 /\left(2 \delta_{1}\right)} \vee 2$ (where $a_{1}\left(T^{-2}\right)$ is the constant in the extended version of (2.1) $)$ so that

$$
\Phi(r) \geq 2 \Phi(r / M), \quad \text { for all } r \leq \operatorname{diam}(D) .
$$

and consider three cases separately:

(1) $\delta_{D}(x) \leq \delta_{D}(y) \leq|x-y| / M$ : Then in case (i), by (2.2), we have

$$
\begin{aligned}
I & =\int_{0}^{\Phi(|x-y|)}\left(1 \wedge \frac{\Phi\left(\delta_{D}(x)\right)}{t}\right)^{1 / 2}\left(1 \wedge \frac{\Phi\left(\delta_{D}(y)\right)}{t}\right)^{1 / 2} \psi\left(t^{-1}\right) d t \\
& \geq \int_{0}^{\Phi\left(\delta_{D}(x)\right)} \psi\left(t^{-1}\right) d t \asymp^{c_{15}} \psi\left(\Phi\left(\delta_{D}(x)\right)^{-1}\right) \Phi\left(\delta_{D}(x)\right),
\end{aligned}
$$

while in case (ii), using (2.2) and (8.10) we have

$$
\begin{aligned}
I & \geq \Phi\left(\delta_{D}(x)\right)^{1 / 2} \Phi\left(\delta_{D}(y)\right)^{1 / 2} \int_{\Phi\left(\delta_{D}(y)\right)}^{\Phi(|x-y|)} t^{-1} \psi\left(t^{-1}\right) d t \\
& \geq \Phi\left(\delta_{D}(x)\right)^{1 / 2} \Phi\left(\delta_{D}(y)\right)^{1 / 2} \int_{\Phi\left(\delta_{D}(y)\right)}^{\Phi\left(M \delta_{D}(y)\right)} t^{-1} \psi\left(t^{-1}\right) d t \\
& \geq \Phi\left(\delta_{D}(x)\right)^{1 / 2} \Phi\left(\delta_{D}(y)\right)^{1 / 2} \int_{\Phi\left(\delta_{D}(y)\right)}^{2 \Phi\left(\delta_{D}(y)\right)} t^{-1} \psi\left(t^{-1}\right) d t \\
& \geq c_{16} \Phi\left(\delta_{D}(x)\right)^{1 / 2} \Phi\left(\delta_{D}(y)\right)^{1 / 2} \psi\left(\Phi\left(\delta_{D}(y)\right)^{-1}\right) .
\end{aligned}
$$


(2) $\delta_{D}(x) \leq|x-y| / M \leq \delta_{D}(y)$ : Then in case (i), using (2.2), we have

$$
I \asymp^{c_{17}} \int_{0}^{\Phi(|x-y|)}\left(1 \wedge \frac{\Phi\left(\delta_{D}(x)\right)}{t}\right)^{1 / 2} \psi\left(t^{-1}\right) d t \geq I_{1} \asymp^{c_{1}} \psi\left(\Phi\left(\delta_{D}(x)\right)^{-1}\right) \Phi\left(\delta_{D}(x)\right),
$$

while in case (ii), using (2.1), (2.2) and the fact that $r^{1 / 2} \psi\left(r^{-1}\right)$ is almost increasing near 0 , we have

$$
\begin{aligned}
& I \geq c_{18} I_{4}=c_{18} \Phi\left(\delta_{D}(x)\right)^{1 / 2} \int_{\Phi\left(\delta_{D}(x)\right)}^{\Phi(|x-y|)} t^{-1 / 2} \psi\left(t^{-1}\right) d t \\
& \geq c_{18} \Phi\left(\delta_{D}(x)\right)^{1 / 2} \int_{\Phi(|x-y| / M)}^{\Phi(|x-y|)} t^{-1}\left(t^{1 / 2} \psi\left(t^{-1}\right)\right) d t \\
& \geq c_{19} \Phi\left(\delta_{D}(x)\right)^{1 / 2} \Phi(|x-y| / M)^{1 / 2} \psi\left(\Phi(|x-y| / M)^{-1}\right) \int_{\Phi(|x-y| / M)}^{\Phi(|x-y|)} t^{-1} d t \\
& \geq c_{20} \log \left(\frac{\Phi(|x-y|)}{\Phi(|x-y| / M)}\right) \Phi\left(\delta_{D}(x)\right)^{1 / 2} \Phi(|x-y|)^{1 / 2} \psi\left(\Phi(|x-y|)^{-1}\right) \\
& \geq c_{21}(\log 2) \Phi\left(\delta_{D}(x)\right)^{1 / 2} \Phi(|x-y|)^{1 / 2} \psi\left(\Phi(|x-y|)^{-1}\right) .
\end{aligned}
$$

Here the last line follows from (8.10).

(3) $|x-y| / M \leq \delta_{D}(x) \leq \delta_{D}(y)$ : Here by (2.1) and (2.2), we have

$$
I \asymp^{c_{22}} \int_{0}^{\Phi(|x-y|)} \psi\left(t^{-1}\right) d t \asymp^{c_{23}} \Phi(|x-y|) \psi\left(\Phi(|x-y|)^{-1}\right) .
$$

We summarize the above calculations as follows:

Case (i): By combining (8.5), (8.7), (8.9), (8.11), (88.13) and (8.15), and using the fact that $r \psi\left(r^{-1}\right)$ is increasing, cf. (2.11), we get that

$$
I \asymp^{c_{24}}\left[\Phi\left(\delta_{D}(x) \wedge \delta_{D}(y)\right) \psi\left(\Phi\left(\delta_{D}(x) \wedge \delta_{D}(y)\right)^{-1}\right)\right] \wedge\left[\Phi(|x-y|) \psi\left(\Phi(|x-y|)^{-1}\right)\right] .
$$

Therefore

$$
\frac{1}{|x-y|^{d} \Phi(|x-y|)} I \asymp^{c_{25}} \frac{\left.\psi\left(\Phi(|x-y|)^{-1}\right)\right)}{|x-y|^{d}}\left(\frac{\Phi\left(\delta_{D}(x) \wedge \delta_{D}(y)\right) \psi\left(\Phi\left(\delta_{D}(x) \wedge \delta_{D}(y)\right)^{-1}\right)}{\left.\Phi(|x-y|) \psi\left(\Phi(|x-y|)^{-1}\right)\right)} \wedge 1\right),
$$

which together with (8.4) proves (i).

Case (ii): By combining (8.6), (8.8), (8.9), (8.12), (8.14) and (8.15) and by using (8.3), we see that

$$
\begin{aligned}
& I \asymp \asymp^{c_{26}}\left[\Phi\left(\delta_{D}(x) \wedge \delta_{D}(y)\right)^{1 / 2} \wedge \Phi(|x-y|)^{1 / 2}\right] \\
& \times\left[\left[\Phi\left(\delta_{D}(x) \vee \delta_{D}(y)\right)^{1 / 2} \psi\left(\Phi\left(\delta_{D}(x) \vee \delta_{D}(y)\right)^{-1}\right)\right] \wedge\left[\Phi(|x-y|)^{1 / 2} \psi\left(\Phi(|x-y|)^{-1}\right)\right]\right] .
\end{aligned}
$$

Thus

$$
\frac{1}{|x-y|^{d} \Phi(|x-y|)} I
$$




$$
\asymp^{c_{27}} \frac{\left.\psi\left(\Phi(|x-y|)^{-1}\right)\right)}{|x-y|^{d}}\left(\frac{\Phi\left(\delta_{D}(x) \wedge \delta_{D}(y)\right)^{1 / 2}}{\Phi(|x-y|)^{1 / 2}} \wedge 1\right)\left(\frac{\eta\left(\delta_{D}(x) \vee \delta_{D}(y)\right)}{\eta(|x-y|)} \wedge 1\right) .
$$

Again, together with (8.4), this gives (ii).

Lemma 8.3 (i) Suppose that $r \mapsto r^{1 / 2} \psi\left(r^{-1}\right)$ is almost decreasing near 0 . Then there exists $C>0$ depending on the diameter of $D$ such that for all $x, y \in D$,

$$
\left(\frac{\Phi\left(\delta_{D}(x)\right)^{1 / 2}}{\Phi(|x-y|)^{1 / 2}} \wedge 1\right)\left(\frac{\Phi\left(\delta_{D}(y)\right)^{1 / 2}}{\Phi(|x-y|)^{1 / 2}} \wedge 1\right) \leq C\left(\frac{\theta\left(\delta_{D}(x) \wedge \delta_{D}(y)\right)}{\theta(|x-y|)} \wedge 1\right) .
$$

(ii) Suppose that $r \mapsto r^{1 / 2} \psi\left(r^{-1}\right)$ is almost increasing near 0 . Then there exists $C>0$ depending on the diameter of $D$ such that for all $x, y \in D$,

$$
\begin{aligned}
& \left(\frac{\Phi\left(\delta_{D}(x)\right)^{1 / 2}}{\Phi(|x-y|)^{1 / 2}} \wedge 1\right)\left(\frac{\Phi\left(\delta_{D}(y)\right)^{1 / 2}}{\Phi(|x-y|)^{1 / 2}} \wedge 1\right) \\
& \leq C\left(\frac{\Phi\left(\delta_{D}(x) \wedge \delta_{D}(y)\right)^{1 / 2}}{\Phi(|x-y|)^{1 / 2}} \wedge 1\right)\left(\frac{\eta\left(\delta_{D}(x) \vee \delta_{D}(y)\right)}{\eta(|x-y|)} \wedge 1\right) .
\end{aligned}
$$

Proof. (i) Denote the left-hand side of (8.16) by $I$ and the right-hand side of (8.16) by $I I$. Without loss of generality we assume that $\delta_{D}(x) \leq \delta_{D}(y)$. We consider three cases:

(1) $\delta_{D}(x) \leq \delta_{D}(y) \leq|x-y|:$ Then

$$
\begin{aligned}
I & =\frac{\Phi\left(\delta_{D}(x)\right)^{1 / 2} \Phi\left(\delta_{D}(y)\right)^{1 / 2}}{\Phi(|x-y|)}=\frac{\Phi\left(\delta_{D}(x)\right) \psi\left(\Phi\left(\delta_{D}(x)\right)^{-1}\right)}{\Phi(|x-y|) \psi\left(\Phi(|x-y|)^{-1}\right)} \frac{\Phi\left(\delta_{D}(y)\right)^{1 / 2} \psi\left(\Phi(|x-y|)^{-1}\right)}{\Phi\left(\delta_{D}(x)\right)^{1 / 2} \psi\left(\Phi\left(\delta_{D}(x)\right)^{-1}\right)} \\
& \leq \frac{\theta\left(\delta_{D}(x)\right)}{\theta(|x-y|)} \frac{\Phi\left(\delta_{D}(y)\right)^{1 / 2} \psi\left(\Phi\left(\delta_{D}(y)\right)^{-1}\right)}{\Phi\left(\delta_{D}(x)\right)^{1 / 2} \psi\left(\Phi\left(\delta_{D}(x)\right)^{-1}\right)} \leq c_{1} \frac{\theta\left(\delta_{D}(x)\right)}{\theta(|x-y|)}=c_{1} I I,
\end{aligned}
$$

where the last inequality follows from the assumption that $r \mapsto^{1 / 2} \psi\left(r^{-1}\right)$ is almost decreasing near 0 .

(2) $\delta_{D}(x) \leq|x-y| \leq \delta_{D}(y):$ Then

$$
\begin{aligned}
I & =\frac{\Phi\left(\delta_{D}(x)\right)^{1 / 2}}{\Phi(|x-y|)^{1 / 2}}=\frac{\Phi\left(\delta_{D}(x)\right) \psi\left(\Phi\left(\delta_{D}(x)\right)^{-1}\right)}{\Phi(|x-y|) \psi\left(\Phi(|x-y|)^{-1}\right)} \frac{\Phi(|x-y|)^{1 / 2} \psi\left(\Phi(|x-y|)^{-1}\right)}{\Phi\left(\delta_{D}(x)\right)^{1 / 2} \psi\left(\Phi\left(\delta_{D}(x)\right)^{-1}\right)} \\
& \leq c_{2} \frac{\theta\left(\delta_{D}(x)\right)}{\theta(|x-y|)}=c_{2} I I,
\end{aligned}
$$

again because $r \mapsto r^{1 / 2} \psi\left(r^{-1}\right)$ is almost decreasing near 0 .

(3) $|x-y| \leq \delta_{D}(x) \leq \delta_{D}(y)$ : Then both $I$ and $I I$ are equal to 1 .

(ii) Again, denote the left-hand side of (8.17) by $I$ and the right-hand side of (8.17) by $I I$, and assume that $\delta_{D}(x) \leq \delta_{D}(y)$.

(1) $\delta_{D}(x) \leq \delta_{D}(y) \leq|x-y|$ : Then

$$
I=\frac{\Phi\left(\delta_{D}(x)\right)^{1 / 2} \Phi\left(\delta_{D}(y)\right)^{1 / 2}}{\Phi(|x-y|)} \leq \frac{\Phi\left(\delta_{D}(x)\right)^{1 / 2}}{\Phi(|x-y|)^{1 / 2}} \frac{\Phi\left(\delta_{D}(y)\right)^{1 / 2}}{\Phi(|x-y|)^{1 / 2}} \frac{\psi\left(\Phi\left(\delta_{D}(y)\right)^{-1}\right)}{\psi\left(\Phi(|x-y|)^{-1}\right)}
$$




$$
=\frac{\Phi\left(\delta_{D}(x)\right)^{1 / 2}}{\Phi(|x-y|)^{1 / 2}} \frac{\eta\left(\delta_{D}(y)\right)}{\eta(|x-y|)}=I I .
$$

(2) $\delta_{D}(x) \leq|x-y| \leq \delta_{D}(y)$ : By the assumption that $r \mapsto r^{1 / 2} \psi\left(r^{-1}\right)$ is almost increasing near 0 , we see that $\eta$ is also almost increasing near 0 . Therefore,

$$
I=\frac{\Phi\left(\delta_{D}(x)\right)^{1 / 2}}{\Phi(|x-y|)^{1 / 2}} \leq c_{3} \frac{\Phi\left(\delta_{D}(x)\right)^{1 / 2}}{\Phi(|x-y|)^{1 / 2}} \frac{\eta\left(\delta_{D}(y)\right)}{\eta(|x-y|)}=c_{3} I I .
$$

(3) $|x-y| \leq \delta_{D}(x) \leq \delta_{D}(y)$ : Now $I=1$, while $I I \geq c$, because $\eta$ is almost increasing near 0 .

Recall that $j(r)=\psi\left(\Phi(r)^{-1}\right) r^{-d}$ for $r>0$.

Theorem 8.4 (i) Suppose that $r \mapsto r^{1 / 2} \psi\left(r^{-1}\right)$ is almost decreasing near 0 and for each $T>0$ there is a constant $C_{4}=C_{4}(T, \psi)>0$ such that (8.2) holds true. Then there exists $C \geq 1$ such that for all $x, y \in D$,

$$
J^{Y^{D}}(x, y) \asymp^{C}\left(\frac{\theta\left(\delta_{D}(x) \wedge \delta_{D}(y)\right)}{\theta(|x-y|)} \wedge 1\right) j(|x-y|) .
$$

(ii) Suppose that $r \mapsto r^{1 / 2} \psi\left(r^{-1}\right)$ is almost increasing near 0 and for every $T>0$, there is a constant $C_{5}=C_{5}(T, \psi)>0$ so that (8.3) holds true. Then there exists $C \geq 1$ such that for all $x, y \in D$,

$$
J^{Y^{D}}(x, y) \asymp C\left(\frac{\Phi\left(\delta_{D}(x) \wedge \delta_{D}(y)\right)^{1 / 2}}{\Phi(|x-y|)^{1 / 2}} \wedge 1\right)\left(\frac{\eta\left(\delta_{D}(x) \vee \delta_{D}(y)\right)}{\eta(|x-y|)} \wedge 1\right) j(|x-y|) .
$$

Proof. Let $T:=2 \Phi(\operatorname{diam}(D))$. By (2.20), (6.1) and (6.2), we have

$$
\begin{aligned}
& J^{Y^{D}}(x, y)=\int_{0}^{\infty} p^{D}(t, x, y) \nu(t) d t \\
& \leq c_{1}\left(\int_{0}^{\Phi(|x-y|)} r(t, x, y) \nu(t) d t+\int_{\Phi(|x-y|)}^{T} r(t, x, y) \nu(t) d t+\int_{T}^{\infty} p^{D}(t, x, y) v(t) d t\right) \\
& =: c_{1}\left(I_{1}+I_{2}+I_{3}\right) .
\end{aligned}
$$

(i) By Lemma 8.1(i), we get

$$
I_{1} \leq c_{2}\left(\frac{\theta\left(\delta_{D}(x) \wedge \delta_{D}(y)\right)}{\theta(|x-y|)} \wedge 1\right) j(|x-y|) .
$$

By using Lemma 6.2 and the fact that $\nu$ is decreasing in the first line, and Lemma $8.3(\mathrm{i})$, the definition of $j$ and (2.5) in the second line,

$$
\begin{aligned}
I_{2} & \leq c_{3}\left(\frac{\Phi\left(\delta_{D}(x)\right)^{1 / 2}}{\Phi(|x-y|)^{1 / 2}} \wedge 1\right)\left(\frac{\Phi\left(\delta_{D}(y)\right)^{1 / 2}}{\Phi(|x-y|)^{1 / 2}} \wedge 1\right) \frac{\Phi(|x-y|) \nu(\Phi(|x-y|)}{|x-y|^{d}} \\
& \leq c_{4}\left(\frac{\theta\left(\delta_{D}(x) \wedge \delta_{D}(y)\right)}{\theta(|x-y|)} \wedge 1\right) j(|x-y|) .
\end{aligned}
$$


By using Lemma 6.1 in the first line, and again Lemma 8.3)(i), the definition of $j$ and (2.5) in the second line, we have

$$
\begin{aligned}
I_{3} & \leq c_{5}\left(\frac{\Phi\left(\delta_{D}(x)\right)^{1 / 2}}{\Phi(|x-y|)^{1 / 2}} \wedge 1\right)\left(\frac{\Phi\left(\delta_{D}(y)\right)^{1 / 2}}{\Phi(|x-y|)^{1 / 2}} \wedge 1\right) j(\mid x-y) \\
& \leq c_{6}\left(\frac{\theta\left(\delta_{D}(x) \wedge \delta_{D}(y)\right)}{\theta(|x-y|)} \wedge 1\right) j(|x-y|) .
\end{aligned}
$$

The three displays above prove the upper bound. For the lower bound, it suffices to use the lower bound for $I_{1}$ coming from Lemma 8.1(i).

(ii) This is proved in the same way as (i) by using part (ii) of Lemmas 8.1 and 8.3 .

Example 8.5 Assume that $\psi(\lambda)=\lambda^{\gamma}$ where $\gamma \in(0,1)$. As already mentioned in Remark 8.2, when $\gamma>1 / 2$ the assumptions in (i) hold true. Since $\theta(t)=\Phi(t)^{1-\gamma}$ we get that

$$
J^{Y^{D}}(x, y) \asymp^{c}\left(\frac{\Phi\left(\delta_{D}(x) \wedge \delta_{D}(y)\right)}{\Phi(|x-y|)} \wedge 1\right)^{1-\gamma} \frac{\Phi(|x-y|)^{-\gamma}}{|x-y|^{d}} .
$$

When $\gamma<1 / 2$, the assumptions in (ii) hold true, $\eta(t)=\Phi(t)^{1 / 2-\gamma}$ and

$$
J^{Y^{D}}(x, y) \asymp^{c}\left(\frac{\Phi\left(\delta_{D}(x) \wedge \delta_{D}(y)\right)}{\Phi(|x-y|)} \wedge 1\right)^{1 / 2}\left(\frac{\Phi\left(\delta_{D}(x) \vee \delta_{D}(y)\right)}{\Phi(|x-y|)} \wedge 1\right)^{1 / 2-\gamma} \frac{\Phi(|x-y|)^{-\gamma}}{|x-y|^{d}} .
$$

The case $\gamma=1 / 2$ is not covered by Theorem 8.4 , but by following the proofs of Lemmas 8.1 and 8.3 step by step, it is straightforward to deduce that

$$
\begin{aligned}
& J^{Y^{D}}(x, y) \\
& \asymp^{c}\left(\frac{\Phi\left(\delta_{D}(x) \wedge \delta_{D}(y)\right)}{\Phi(|x-y|)} \wedge 1\right)^{1 / 2} \log \left(1+\frac{\Phi\left(\delta_{D}(x) \vee \delta_{D}(y)\right) \wedge \Phi(|x-y|)}{\Phi\left(\delta_{D}(x) \wedge \delta_{D}(y)\right) \wedge \Phi(|x-y|)}\right) \frac{\Phi(|x-y|)^{-1 / 2}}{|x-y|^{d}} .
\end{aligned}
$$

In particular, with $y \in D$ fixed, as $\delta_{D}(x) \rightarrow 0$, we have

$$
J^{Y^{D}}(x, y) \asymp^{c} \begin{cases}\Phi\left(\delta_{D}(x)\right)^{1 / 2}, & 0<\gamma<1 / 2, \\ \Phi\left(\delta_{D}(x)\right)^{1 / 2} \log \left(1 / \Phi\left(\delta_{D}(x)\right)\right), & \gamma=1 / 2, \\ \Phi\left(\delta_{D}(x)\right)^{1 / 2} \Phi\left(\delta_{D}(x)\right)^{1 / 2-\gamma}, & 1 / 2<\gamma<1 .\end{cases}
$$

\section{$9 \quad$ Failure of BHP in the case of $\gamma_{2} \leq 1 / 2$}

In this section we assume that $d \geq 2$ and that $D$ is a bounded $C^{1,1}$ open set in $\mathbb{R}^{d}$ with $C^{1,1}$ characteristics $(R, \Lambda)$ with $R<1$. The goal of this section is to give an example showing that even the non-scale invariant boundary Harnack principle does not hold when $\gamma_{2} \leq 1 / 2$. For simplicity we consider the case $\psi(t)=t^{\gamma}$ and $\gamma_{2}=\gamma \leq 1 / 2$. The example works for any bounded $C^{1,1}$ open set. 
Suppose that the non-scale invariant boundary Harnack principle holds near the boundary of $D$. That is, there is a constant $\widehat{R} \in(0,1)$ such that for any $r \in(0, \widehat{R}]$, there exists a constant $c=c(r) \geq 1$ such that for every $Q \in \partial D$ and any non-negative functions $f, g$ in $D$ which are harmonic in $D \cap B(Q, r)$ with respect to $Y^{D}$ and vanish continuously on $\partial D \cap B(Q, r)$, we have

$$
\frac{f(x)}{f(y)} \leq c \frac{g(x)}{g(y)} \quad \text { for all } x, y \in D \cap B(Q, r / 2) .
$$

Note that we can take $g(\cdot)=G^{Y^{D}}(\cdot, w)$ with $w \notin D \cap B(Q, r)$. Thus by Theorem 6.4, we have that for any $r \in(0, \widehat{R}]$ there exists a constant $C_{6}=C_{6}(r)>0$ such that for every $Q \in \partial D$ and any non-negative function $f$ in $D$ which is harmonic in $D \cap B(Q, r)$ with respect to $Y^{D}$ and vanishes continuously on $\partial D \cap B(Q, r)$,

$$
\frac{f(x)}{f(y)} \leq C_{6} \frac{\Phi\left(\delta_{D}(x)\right)^{1 / 2}}{\Phi\left(\delta_{D}(y)\right)^{1 / 2}}, \quad \text { for all } x, y \in D \cap B(Q, r / 2) .
$$

Fix $Q \in \partial D$. We choose a $C^{1,1}$ function $\varphi: \mathbb{R}^{d-1} \rightarrow \mathbb{R}$ satisfying $\varphi(\widetilde{0})=0, \nabla \varphi(\widetilde{0})=$ $(0, \ldots, 0),\|\nabla \varphi\|_{\infty} \leq \Lambda,|\nabla \varphi(\widetilde{y})-\nabla \varphi(\widetilde{z})| \leq \Lambda|\widetilde{y}-\widetilde{z}|$, and an orthonormal coordinate system $C S$ with its origin at $Q$ such that

$$
B(Q, R) \cap D=\left\{y=\left(\widetilde{y}, y_{d}\right) \in B(0, R) \text { in } C S: y_{d}>\varphi(\widetilde{y})\right\}
$$

Recall that $\kappa_{0}=\left(1+(1+\Lambda)^{2}\right)^{-1 / 2}$. Since $D$ satisfies the interior ball condition, there exist $r_{0} \leq \widehat{R} \wedge\left(2^{-4} \kappa_{0} R\right)$ and $x^{(1)} \in B(Q, R) \cap D$ with $\delta_{D}\left(x^{(1)}\right)=r_{0}$ such that $\delta_{D}\left(x^{(s)}\right)=\left|x^{(s)}-Q\right|$ for all $s \leq 1$ where $x^{(s)}=Q+s\left(x^{(1)}-Q\right)$. define

In the coordinate system $C S$ we have $\widetilde{x}^{(s)}=\widetilde{0}$ and $x^{(1)}=\left(\widetilde{0}, r_{0}\right)$. For any $b_{1}, b_{2}>0$, we

$$
D^{*}\left(b_{1}, b_{2}\right):=\left\{y=\left(\widetilde{y}, y_{d}\right) \text { in } C S: 0<y_{d}-\varphi(\widetilde{y})<2^{-2} \kappa_{0} r_{0} b_{1},|\widetilde{y}|<2^{-2} \kappa_{0} r_{0} b_{2}\right\} .
$$

By (6.12), we have that $D^{*}(2,2) \subset D \cap B\left(Q, r_{0} / 2\right)$. Recall that $V_{Q}\left(2^{-2} \kappa_{0} r_{0}\right)$ is a $C^{1,1}$ domain with $C^{1,1}$ characteristics $\left(r_{0} R / L, \Lambda L / r_{0}\right)$ such that $D^{*}(1 / 2,1 / 2) \subset V_{Q}\left(2^{-2} \kappa_{0} r_{0}\right) \subset D^{*}(1,1)$, where $L=L(R, \Lambda, d)>0$. Let $V=V_{Q}\left(2^{-2} \kappa_{0} r_{0}\right)$ and $U=D^{*}(2,2)$.

Recall that

$$
g(r)=\frac{1}{r^{d} \psi\left(\Phi(r)^{-1}\right)}=\frac{\Phi(r)^{\gamma}}{r^{d}} .
$$

Lemma 9.1 If $\gamma \leq 1 / 2$, then there exists $C>0$ such that

$$
\mathbb{E}_{x}\left[\int_{0}^{\tau_{V}} \Phi\left(\delta_{D}\left(Y_{t}^{D}\right)\right)^{\frac{1}{2}-\gamma} d t\right] \geq C \Phi\left(\delta_{D}(x)\right)^{1 / 2} \log \left(r_{0} / \delta_{D}(x)\right)
$$

for all $x=x^{(s)}=(\widetilde{0}, s)$ in $C S$ with $s \in\left(0,2^{-7} \kappa_{0} r_{0}\right)$. 
Proof. By Proposition 6.5,

$$
\begin{aligned}
& \mathbb{E}_{x}\left[\int_{0}^{\tau_{V}} \Phi\left(\delta_{D}\left(Y_{t}^{D}\right)\right)^{\frac{1}{2}-\gamma} d t\right]=\int_{V} G_{V}^{Y^{D}}(x, z) \Phi\left(\delta_{D}(z)\right)^{\frac{1}{2}-\gamma} d z \\
& \geq \int_{V} G^{Y^{V}}(x, z) \Phi\left(\delta_{D}(z)\right)^{\frac{1}{2}-\gamma} d z \\
& \geq c_{1} \int_{V} \Phi\left(\delta_{D}(z)\right)^{\frac{1}{2}-\gamma}\left(\frac{\Phi\left(\delta_{V}(x)\right)^{1 / 2}}{\Phi(|x-z|)^{1 / 2}} \wedge 1\right)\left(\frac{\Phi\left(\delta_{V}(z)\right)^{1 / 2}}{\Phi(|x-z|)^{1 / 2}} \wedge 1\right) g(|x-z|) d z .
\end{aligned}
$$

Recall from (17.3) that there exists $\varepsilon \in\left(0,(2(1+\Lambda))^{-2}\right)$ such that the cone

$$
\mathcal{C}_{\varepsilon}(x):=\left\{y=\left(\widetilde{y}, y_{d}\right) \in B\left(x, 2^{-6} \kappa_{0} r_{0}\right) \text { in } C S: y_{d}>x_{d},|\widetilde{y}|<\varepsilon\left(y_{d}-x_{d}\right)\right\}
$$

is contained in $D^{*}\left(2^{-2}, 2^{-2}\right)$. Moreover, (7.4) implies

$$
\left(\frac{\Phi\left(\delta_{V}(z)\right)}{\Phi(|x-z|)} \wedge 1\right) \asymp 1, \quad z \in \mathcal{C}_{\varepsilon / 2}(x) .
$$

If $z \in \mathcal{C}_{\varepsilon / 2}(x) \backslash B\left(x, \delta_{D}(x) / 2\right)$, then $|x-z| \geq \delta_{D}(x) / 2$, so by (7.4) and (2.9),

$$
\left(\frac{\Phi\left(\delta_{D}(x)\right)}{\Phi(|x-z|)} \wedge 1\right) \Phi\left(\delta_{D}(z)\right)^{1-2 \gamma} \geq c_{2}\left(\frac{\Phi\left(\delta_{D}(x)\right)}{\Phi(|x-z|)} \wedge 1\right) \Phi(|x-z|)^{1-2 \gamma} \geq c_{3} \frac{\Phi\left(\delta_{D}(x)\right)}{\Phi(|x-z|)^{2 \gamma}} .
$$

Therefore, using (9.3),

$$
\begin{aligned}
& \int_{V} \Phi\left(\delta_{D}(z)\right)^{\frac{1}{2}-\gamma}\left(\frac{\Phi\left(\delta_{V}(x)\right)^{1 / 2}}{\Phi(|x-z|)^{1 / 2}} \wedge 1\right)\left(\frac{\Phi\left(\delta_{V}(z)\right)^{1 / 2}}{\Phi(|x-z|)^{1 / 2}} \wedge 1\right) g(|x-z|) d z \\
& \geq c_{4} \Phi\left(\delta_{D}(x)\right)^{1 / 2} \int_{\mathcal{C}_{\varepsilon / 2}(x) \backslash B\left(x, \delta_{D}(x) / 2\right)} \frac{g(|x-z|)}{\Phi(|x-z|)^{\gamma}} d z \\
& \geq c_{5} \Phi\left(\delta_{D}(x)\right)^{1 / 2} \int_{\delta_{D}(x) / 2}^{2^{-6} \kappa_{0} r_{0}} \frac{1}{s} d s \geq c_{6} \Phi\left(\delta_{D}(x)\right)^{1 / 2} \log \left(r_{0} / \delta_{D}(x)\right) .
\end{aligned}
$$

Choose a point $z_{0} \in \partial D \backslash \overline{D \cap B\left(Q, 2 r_{0}\right)}$ with $\left|z_{0}-Q\right| \leq 1$ (such $z_{0}$ exists since $2 r_{0} \leq$ $\left.2^{-3} \kappa_{0} R\right)$. For $n \in \mathbb{N}$ large enough so that $B\left(z_{0}, 1 / n\right)$ does not intersect $B\left(Q, 2 r_{0}\right)$, we define

$$
f_{n}(y):=\Phi\left(\delta_{D}(y)\right)^{-1 / 2} \mathbf{1}_{D \cap B\left(z_{0}, 1 / n\right)}(y) \times \begin{cases}\left|D \cap B\left(z_{0}, 1 / n\right)\right|^{-1}, & \text { for } \gamma<1 / 2 \\ K_{n}^{-1}, & \text { for } \gamma=1 / 2,\end{cases}
$$

where

$$
K_{n}:=\int_{D \cap B\left(z_{0}, 1 / n\right)} \log \left(\frac{1}{\Phi\left(\delta_{D}(y)\right)}\right) d y
$$

Define

$$
g_{n}(x):=\mathbb{E}_{x}\left[f_{n}\left(Y_{\tau_{V}}^{D}\right)\right]=\int_{D \backslash U} \int_{V} G_{V}^{Y^{D}}(x, z) J^{Y^{D}}(z, y) f_{n}(y) d z d y, \quad x \in V .
$$


Lemma 9.2 If $\gamma \leq 1 / 2$, then there exists $C_{7}>0$ such that

$$
\liminf _{n \rightarrow \infty} g_{n}(x) \geq C_{7} \Phi\left(\delta_{D}(x)\right)^{1 / 2} \log \left(r_{0} / \delta_{D}(x)\right)
$$

for all $x=x^{(s)}=(\widetilde{0}, s)$ in $C S$ with $s \in\left(0,2^{-7} \kappa_{0} r_{0}\right)$.

Proof. (i) Case $\gamma<1 / 2$ : Since

$$
1 \asymp^{c}|z-y| \geq c_{1} \delta_{D}(z) \quad \text { and } \quad|y-z| \geq c_{2} \delta_{D}(y), \quad \text { for }(y, z) \in\left(D \cap B\left(z_{0}, 1 / n\right)\right) \times V
$$

using $(8.18)$ we have

$J^{Y^{D}}(z, y) \asymp^{c} \Phi\left(\delta_{D}(y)\right)^{1 / 2} \Phi\left(\delta_{D}(z)\right)^{1 / 2} \Phi\left(\delta_{D}(z) \vee \delta_{D}(y)\right)^{-\gamma} \quad$ for $(y, z) \in\left(D \cap B\left(z_{0}, 1 / n\right)\right) \times V$.

Thus,

$$
\begin{aligned}
& g_{n}(x)=\int_{D \backslash U} \int_{V} G_{V}^{Y^{D}}(x, z) J^{Y^{D}}(z, y) f_{n}(y) d z d y \\
& \asymp^{c}\left|D \cap B\left(z_{0}, 1 / n\right)\right|^{-1} \int_{V} \int_{D \cap B\left(z_{0}, 1 / n\right)} \Phi\left(\delta_{D}(z) \vee \delta_{D}(y)\right)^{-\gamma} d y \Phi\left(\delta_{D}(z)\right)^{1 / 2} G_{V}^{Y^{D}}(x, z) d z .
\end{aligned}
$$

Since

$$
\begin{gathered}
\lim _{n \rightarrow \infty}\left|D \cap B\left(z_{0}, 1 / n\right)\right|^{-1} \int_{D \cap B\left(z_{0}, 1 / n\right)} \Phi\left(\delta_{D}(z) \vee \delta_{D}(y)\right)^{-\gamma} d y=\Phi\left(\delta_{D}(z)\right)^{-\gamma}, \quad z \in V, \\
\left|D \cap B\left(z_{0}, 1 / n\right)\right|^{-1} \int_{D \cap B\left(z_{0}, 1 / n\right)} \Phi\left(\delta_{D}(z) \vee \delta_{D}(y)\right)^{-\gamma} d y \leq \Phi\left(\delta_{D}(z)\right)^{-\gamma}, \quad z \in V
\end{gathered}
$$

and

$$
\int_{V} \Phi\left(\delta_{D}(z)\right)^{\frac{1}{2}-\gamma} G_{V}^{Y^{D}}(x, z) d z<\infty
$$

by the dominated convergence theorem, for all $x=x^{(s)}=(\widetilde{0}, s)$ in $C S$ with $s \in\left(0,2^{-7} \kappa_{0} r_{0}\right)$,

$$
\begin{aligned}
& \lim _{n \rightarrow \infty}\left|D \cap B\left(z_{0}, 1 / n\right)\right|^{-1} \int_{V} \int_{D \cap B\left(z_{0}, 1 / n\right)} \Phi\left(\delta_{D}(z) \vee \delta_{D}(y)\right)^{-\gamma} d y \Phi\left(\delta_{D}(z)\right)^{1 / 2} G_{V}^{Y^{D}}(x, z) d z \\
& =\int_{V} \Phi\left(\delta_{D}(z)\right)^{\frac{1}{2}-\gamma} G_{V}^{Y^{D}}(x, z) d z .
\end{aligned}
$$

Combining (9.7) with Lemma 9.1 we conclude that (9.5) holds true for $\gamma<1 / 2$.

(ii) Case $\gamma=1 / 2$ : Using (8.19) and following the same argument in (i), we have

$$
\begin{aligned}
& g_{n}(x)=\int_{D \backslash U} \int_{V} G_{V}^{Y^{D}}(x, z) J^{Y^{D}}(z, y) f_{n}(y) d z d y \\
& \asymp^{c} K_{n}^{-1} \int_{V} \int_{D \cap B\left(z_{0}, 1 / n\right)}\left(\frac{\Phi\left(\delta_{D}(z) \wedge \delta_{D}(y)\right)}{\Phi\left(\delta_{D}(y)\right)}\right)^{1 / 2} \log \left(1+\frac{\Phi\left(\delta_{D}(y) \vee \delta_{D}(z)\right)}{\Phi\left(\delta_{D}(y) \wedge \delta_{D}(z)\right)}\right) d y G_{V}^{Y^{D}}(x, z) d z .
\end{aligned}
$$


We have $\int_{V} G_{V}^{Y^{D}}(x, z) d z<\infty$ and

$$
\begin{aligned}
& K_{n}^{-1} \int_{D \cap B\left(z_{0}, 1 / n\right)}\left(\frac{\Phi\left(\delta_{D}(z) \wedge \delta_{D}(y)\right)}{\Phi\left(\delta_{D}(y)\right)}\right)^{1 / 2} \log \left(1+\frac{\Phi\left(\delta_{D}(y) \vee \delta_{D}(z)\right)}{\Phi\left(\delta_{D}(y) \wedge \delta_{D}(z)\right)}\right) d y \\
\leq & K_{n}^{-1} \int_{D \cap B\left(z_{0}, 1 / n\right) \cap\left\{\delta_{D}(z) \leq \delta_{D}(y)\right\}}\left(\frac{\Phi\left(\delta_{D}(z)\right)}{\Phi\left(\delta_{D}(y)\right)}\right)^{1 / 2} \log \left(1+\frac{\Phi\left(\delta_{D}(y)\right)}{\Phi\left(\delta_{D}(z)\right)}\right) d y \\
& +K_{n}^{-1} \int_{D \cap B\left(z_{0}, 1 / n\right) \cap\left\{\delta_{D}(z)>\delta_{D}(y)\right\}} \log \left(1+\frac{\Phi\left(\delta_{D}(z)\right)}{\Phi\left(\delta_{D}(y)\right)}\right) d y \\
\leq & c K_{n}^{-1} \int_{D \cap B\left(z_{0}, 1 / n\right)} \log \left(\frac{1}{\Phi\left(\delta_{D}(y)\right)}\right) d y \leq c, \quad z \in V .
\end{aligned}
$$

Moreover, since

$\limsup _{n \rightarrow \infty} K_{n}^{-1} \int_{D \cap B\left(z_{0}, 1 / n\right)} \log \left(1+\frac{\Phi\left(\delta_{D}(y)\right)}{\Phi\left(\delta_{D}(z)\right)}\right) d y \leq \limsup _{n \rightarrow \infty} K_{n}^{-1}\left|D \cap B\left(z_{0}, 1 / n\right)\right|=0, \quad z \in V$, for each $z \in V$,

$$
\begin{aligned}
& \lim _{n \rightarrow \infty} K_{n}^{-1} \int_{D \cap B\left(z_{0}, 1 / n\right)}\left(\frac{\Phi\left(\delta_{D}(z) \wedge \delta_{D}(y)\right)}{\Phi\left(\delta_{D}(y)\right)}\right)^{1 / 2} \log \left(1+\frac{\Phi\left(\delta_{D}(y) \vee \delta_{D}(z)\right)}{\Phi\left(\delta_{D}(y) \wedge \delta_{D}(z)\right)}\right) d y \\
= & \lim _{n \rightarrow \infty} K_{n}^{-1} \int_{D \cap B\left(z_{0}, 1 / n\right)} \log \left(1+\frac{\Phi\left(\delta_{D}(z)\right)}{\Phi\left(\delta_{D}(y)\right)}\right) d y \\
= & \lim _{n \rightarrow \infty} K_{n}^{-1} \int_{D \cap B\left(z_{0}, 1 / n\right)} \log \left(1+\frac{\Phi\left(\delta_{D}(y)\right)}{\Phi\left(\delta_{D}(z)\right)}\right) d y \\
& +\lim _{n \rightarrow \infty} K_{n}^{-1} \int_{D \cap B\left(z_{0}, 1 / n\right)}\left(\log \frac{1}{\Phi\left(\delta_{D}(y)\right)}-\log \frac{1}{\Phi\left(\delta_{D}(z)\right)}\right) d y \\
= & 1-\log \frac{1}{\Phi\left(\delta_{D}(z)\right)} \lim _{n \rightarrow \infty} K_{n}^{-1}\left|D \cap B\left(z_{0}, 1 / n\right)\right|=1 .
\end{aligned}
$$

Thus by the dominated convergence theorem, for all $x=x^{(s)}=(\widetilde{0}, s)$ in $C S$ with $s \in$ $\left(0,2^{-7} \kappa_{0} r_{0}\right)$,

$$
\begin{aligned}
& \lim _{n \rightarrow \infty} K_{n}^{-1} \int_{V} \int_{D \cap B\left(z_{0}, 1 / n\right)}\left(\frac{\Phi\left(\delta_{D}(z) \wedge \delta_{D}(y)\right)}{\Phi\left(\delta_{D}(y)\right)}\right)^{1 / 2} \log \left(1+\frac{\Phi\left(\delta_{D}(y) \vee \delta_{D}(z)\right)}{\Phi\left(\delta_{D}(y) \wedge \delta_{D}(z)\right)}\right) d y G_{V}^{Y^{D}}(x, z) d z \\
& =\int_{V} G_{V}^{Y^{D}}(x, z) d z .
\end{aligned}
$$

Combining (9.9) with Lemma 9.1 we conclude that (9.5) holds true for $\gamma=1 / 2$.

By Lemmas 6.7 and 6.8, we have that for large $n$ anf $y \in D \cap B\left(Q, 2^{-7} \kappa_{0} r_{0}\right)$

$$
g_{n}(y) \leq c_{1} \int_{V} \Phi\left(\delta_{D}(z)\right)^{\frac{1}{2}-\gamma} G_{V}^{Y^{D}}(y, z) d z
$$




$$
\begin{aligned}
& \leq c_{2} \Phi\left(r_{0}\right)^{\frac{1}{2}-\gamma} \int_{D} G^{Y^{D}}(y, z) d z \\
& \leq c_{3} \Phi\left(r_{0}\right)^{\frac{1}{2}-\gamma} \Phi\left(\delta_{D}(y)\right)^{\gamma} \begin{cases}1, & \text { for } \gamma<1 / 2 \\
\log \left(1 / \delta_{D}(y)\right), & \text { for } \gamma=1 / 2\end{cases}
\end{aligned}
$$

Thus we see that $g_{n}$ 's are non-negative functions in $D$ which are harmonic in $D \cap B\left(Q, 2^{-7} \kappa_{0} r_{0}\right)$ with respect to $Y^{D}$ and vanish continuously on $\partial D \cap B\left(Q, 2^{-7} \kappa_{0} r_{0}\right)$. Therefore, by (9.2),

$$
\frac{g_{n}(y)}{g_{n}(w)} \leq C_{6} \frac{\Phi\left(\delta_{D}(y)\right)^{1 / 2}}{\Phi\left(\delta_{D}(w)\right)^{1 / 2}} \quad \text { for all } y \in D \cap B\left(Q, 2^{-8} \kappa_{0} r_{0}\right)
$$

where $w=\left(\widetilde{0}, 2^{-9} \kappa_{0} r_{0}\right)$ and $C_{6}=C_{6}\left(2^{-7} \kappa_{0} r_{0}\right)$. Thus by (9.10), for all $y \in D \cap B\left(Q, 2^{-8} \kappa_{0} r_{0}\right)$,

$$
\limsup _{n \rightarrow \infty} g_{n}(y) \leq C_{6} \limsup _{n \rightarrow \infty} g_{n}(w) \frac{\Phi\left(\delta_{D}(y)\right)^{1 / 2}}{\Phi\left(\delta_{D}(w)\right)^{1 / 2}} \leq c_{4} \log \left(c_{5} / r_{0}\right) \Phi\left(\delta_{D}(y)\right)^{1 / 2} .
$$

This and (9.5) imply that for all $x=x^{(s)}=(\widetilde{0}, s)$ in $C S$ with $s \in\left(0,2^{-8} \kappa_{0} r_{0}\right)$,

$$
\log \left(r_{0} / \delta_{D}(x)\right) \leq\left(c_{4} / C_{7}\right) \log \left(c_{5} / r_{0}\right)
$$

which gives a contradiction.

Acknowledgements: We thank the referee for carefully reading the manuscript and providing some useful suggestions.

\section{References}

[1] Z.-Q. Chen. Gaugeability and conditional gaugeability. Trans. Amer. Math. Soc. 354 (2002), 4639-4679

[2] Z.-Q. Chen, P. Kim and T. Kumagai. On heat kernel estimates and parabolic Harnack inequality for jump processes on metric measure spaces. Acta Math. Sin. 25 (2009), 1067-1086.

[3] Z.-Q. Chen and P. Kim. Global Dirichlet heat kernel estimates for symmetric Lévy processes in half-space. Acta Appl. Math. 146(1) (2016), 113-143.

[4] Z.-Q. Chen, P. Kim and R. Song. Dirichlet heat kernel estimates for fractional Laplacian with gradient perturbation. Ann. Probab. 40 (2012), 2483-2538.

[5] Z.-Q. Chen, P. Kim and R. Song. Dirichlet heat kernel estimates for rotationally symmetric Lévy processes. Proc. Lond. Math. Soc. 109 (2014), 90-120.

[6] Z.-Q. Chen and R. Song. Conditional gauge theorem for non-local Feynman-Kac transforms. Probab. Theory Rel. Fields 125 (2003), 45-72.

[7] M. Fukushima, Y. Oshima and M. Takeda. Dirichlet Forms and Symmetric Markov Processes. Walter De Gruyter, Berlin, 1994. 
[8] Q.-Y. Guan. Boundary Harnack inequality for regional fractional Laplacian. Preprint, 2007. arXiv:0705.1614v3

[9] P. Kim and A. Mimica. Green function estimates for subordinate Brownian motions: stable and beyond. Trans. Amer. Math. Soc. 366 (2014), 4383-4422.

[10] K. Kaleta, P. Sztonyk: Estimates of transition densities and their derivatives for jump Lévy processes, J. Math. Anal. Appl. 431(1) (2015), 260-282.

[11] P. Kim and R. Song. Potential theory of truncated stable processes. Math. Z. 256 (2007), 139-173.

[12] P. Kim, R. Song and Z. Vondraček. Uniform boundary Harnack principle for rotationally symmetric Lévy processes in general open sets. Science China Math. 55 (2012), 2317-2333.

[13] P. Kim, R. Song and Z. Vondraček. Potential theory of subordinate Brownian motions with Gaussian components. Stochastic Process. Appl. 123 (2013), 764-795

[14] P. Kim, R. Song and Z. Vondraček. Global uniform boundary Harnack principle with explicit decay rate and its application. Stochastic Process. Appl. 124 (2014), 235-267.

[15] P. Kim, R. Song and Z. Vondraček. Minimal thinness with respect to subordinate killed Brownian motions. Stoch. Processes Appl. 126 (2016), 1226-1263.

[16] P. Kim, R. Song and Z. Vondraček: Minimal thinness with respect to symmetric Lévy processes. Trans. Amer. Math. Soc. 368(12) (2016), 8785-8822.

[17] P. Kim, R. Song and Z. Vondraček: Scale invariant boundary Harnack principle at infinity for Feller processes. Potential Anal. 47 (2017), 337-367.

[18] P. Kim, R. Song and Z. Vondraček. Potential theory of subordinate killed Brownian motion. Trans. Amer. Math. Soc. 371 (2019), 3917-3969.

[19] P. Kim, R. Song and Z. Vondraček. Heat kernels of non-symmetric jump processes: beyond the stable case. Potential Anal. 49 (2018), 37-90.

[20] M. Kwaśnicki. Ten equivalent definitions of the fractional Laplace operator. Frac. Calc. Appl. Anal. 20(1) (2017), 7-51.

[21] K.-I. Sato. Lévy Processes and Infinitely Divisible Distributions. Cambridge University Press, Cambridge, 1999.

[22] R. Schilling, R. Song and Z. Vondraček. Bernstein Functions: Theory and Applications. 2nd ed., de Gruyter, 2012.

[23] R. Song. Estimates on the Dirichlet heat kernel of domains above the graphs of bounded $C^{1,1}$ functions. Glas. Mat. 39 (2004), 273-286.

[24] R. Song and Z. Vondraček. Potential theory of subordinate killed Brownian motion in a domain. Probab. Theory Related Fields 125 (2003), 578-592.

[25] R. Song and Z. Vondraček. Potential theory of special subordinators and subordinate killed stable processes. J. Theoret. Probab. 19 (2006), 817-847. 
[26] R. Song and Z. Vondraček. On the relationship between subordinate killed and killed subordinate processes. Elect. Comm. Probab. 23 (2008), 325-336.

[27] P. Sztonyk. On harmonic measure for Lévy processes. Probab. Math. Statist. 20 (2000), 383390.

\section{Panki Kim}

Department of Mathematical Sciences and Research Institute of Mathematics, Seoul National University, Building 27, 1 Gwanak-ro, Gwanak-gu Seoul 151-747, Republic of Korea

E-mail: pkim@snu.ac.kr

\section{Renming Song}

Department of Mathematics, University of Illinois, Urbana, IL 61801, USA, and School of Mathematical Sciences, Nankai University, Tianjin 300071, PR China

E-mail: rsong@illinois.edu

\section{Zoran Vondraček}

Department of Mathematics, Faculty of Science, University of Zagreb, Zagreb, Croatia

E-mail: vondra@math.hr 